\title{
Synthesis of Highly Substituted Furans by the Electrophile-Induced Coupling of 2-(1-Alkynyl)-2-alken-1-ones and Nucleophiles
}

\author{
Tuanli Yao, Xiaoxia Zhang and Richard C. Larock* \\ Department of Chemistry, Iowa State University, Ames, Iowa 50011 \\ larock@iastate.edu
}

$\begin{array}{lc}\text { General } & \text { Page } \\ \text { Preparation of 2-(1-Alkynyl)-2-alken-1-ones } & \mathrm{S} 2-\mathrm{S} 2 \\ \mathrm{AuCl}_{3} \text {-catalyzed cyclizations } & \mathrm{S} 2-\mathrm{S} 7 \\ \text { iodine-induced cyclizations } & \mathrm{S} 7-\mathrm{S} 13 \\ \text { PhSeCl-induced cyclizations } & \mathrm{S} 13-\mathrm{S} 26 \\ \text { Elaboration of furan products } & \mathrm{S} 26-\mathrm{S} 27 \\ \text { References } & \mathrm{S} 27-\mathrm{S} 31 \\ \text { Copies of }{ }^{1} \mathrm{H} \text { and }{ }^{13} \mathrm{C} \text { NMR spectra } & \mathrm{S} 31-\mathrm{S} 32\end{array}$


General procedures. All ${ }^{1} \mathrm{H}$ and ${ }^{13} \mathrm{C}$ NMR spectra were recorded at 300 and 75.5 MHz. Thin-layer chromatography was performed using commercially prepared 60-mesh silica gel plates (Whatman K6F), and visualization was effected with short wavelength UV light (254 nm) and a basic $\mathrm{KMnO}_{4}$ solution [3 $\mathrm{g}$ of $\mathrm{KMnO}_{4}+20 \mathrm{~g}$ of $\mathrm{K}_{2} \mathrm{CO}_{3}+5 \mathrm{~mL}$ of $\mathrm{NaOH}(5 \%)+300 \mathrm{~mL}$ of $\mathrm{H}_{2} \mathrm{O}$ ]. All melting points are uncorrected. High resolution mass spectra were recorded on a Kratos MS50TC double focusing magnetic sector mass spectrometer using EI at $70 \mathrm{eV}$. Compounds $1,2,16,17,29,30,36,38,40,47,49,54$, $56,60,62,63,65,66,69$ and 70 have been previously reported. ${ }^{1}$

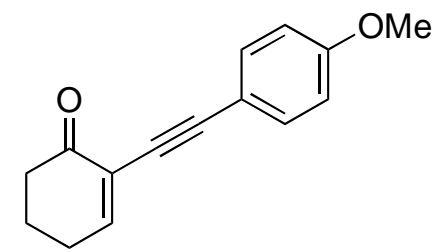

8

2-(4-Methoxyphenyl)ethynyl-2-cyclohexen-1-one (8). This 2-(1-alkynyl)-2-alken-1one was prepared from 2-iodo-2-cyclohexen-1-one ${ }^{2}$ by following a procedure from the literature. ${ }^{3}$ 2-Iodo-2-cyclohexen-1-one (444 mg, 2.0 mmol), $\mathrm{PdCl}_{2}\left(\mathrm{PPh}_{3}\right)_{2}(70.2 \mathrm{mg}, 0.05$ equiv), (4-methoxyphenyl)ethyne (529 mg, 2.0 equiv) and CuI (38 mg, 0.1 equiv) were taken up in THF $(14 \mathrm{~mL})$ at $0{ }^{\circ} \mathrm{C}$. Diisopropylamine ( $0.84 \mathrm{~mL}, 3.0$ equiv) was added, and the resulting mixture was stirred at $0{ }^{\circ} \mathrm{C}$ for $45 \mathrm{~min}$. The mixture was diluted with $\mathrm{Et}_{2} \mathrm{O}(100 \mathrm{~mL})$ and washed with $1 \mathrm{M} \mathrm{HCl}(50 \mathrm{~mL})$ and brine $(20 \mathrm{~mL})$. The organic layer was dried over $\mathrm{MgSO}_{4}$, filtered and concentrated. The residue was purified by flash column chromatography (silica gel, 3:1 hexane/EtOAc) to afford $301 \mathrm{mg}(67 \%)$ of the indicated compound 8 as light yellow solid: $\mathrm{mp} 70-71{ }^{\circ} \mathrm{C} ;{ }^{1} \mathrm{H}$ NMR $\left(\mathrm{CDCl}_{3}\right) \delta$ 2.03-2.10 (m, 2H), 2.46-2.55 (m, 4H), 3.80 (s, 3H), 6.83 (dt, $J=8.4,2.1 \mathrm{~Hz}, 2 \mathrm{H}$ ), 7.32 (t, $J=4.5$ 
$\mathrm{Hz}, 1 \mathrm{H}), 7.43(\mathrm{dt}, J=9.0,2.1 \mathrm{~Hz}, 2 \mathrm{H}) ;{ }^{13} \mathrm{C} \mathrm{NMR}\left(\mathrm{CDCl}_{3}\right) \delta 22.7,26.7,38.4,55.5,82.7$, 92.4, 114.1, 115.2, 125.7, 133.5, 153.8, 159.9, 196.0; $\mathrm{IR}\left(\mathrm{CH}_{2} \mathrm{Cl}_{2}\right)$ 2952, 1688, 1510, $1249 \mathrm{~cm}^{-1}$; HRMS m/z 226.0996 (calcd for $\mathrm{C}_{15} \mathrm{H}_{14} \mathrm{O}_{2}$, 226.0994).

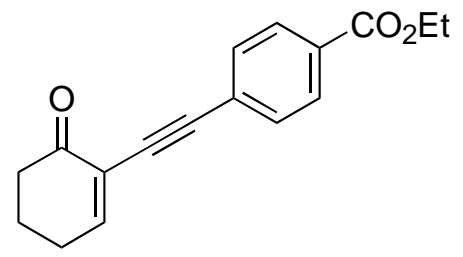

11

Ethyl 4-[(6-oxocyclohexenyl)ethynyl]benzoate (11). This 2-(1-alkynyl)-2-alken-1one was prepared from 2-iodo-2-cyclohexen-1-one ${ }^{2}$ by following the same procedure as that used for compound 8. 2-Iodo-2-cyclohexen-1-one (222 mg, $1.0 \mathrm{mmol}$ ), ethyl 4ethynylbenzoate (348 mg, 2.0 equiv), $\mathrm{PdCl}_{2}\left(\mathrm{PPh}_{3}\right)_{2}$ (35.6 mg, 0.05 equiv), $\mathrm{CuI}$ (20 mg, 0.1 equiv) and diisopropylamine ( $0.42 \mathrm{~mL}, 3.0$ equiv) afforded, after purification by flash column chromatography (silica gel, 3:1 hexane/EtOAc), $160 \mathrm{mg}$ (60\%) of the indicated compound 11 as a yellow solid: mp $116-117{ }^{\circ} \mathrm{C} ;{ }^{1} \mathrm{H}$ NMR $\left(\mathrm{CDCl}_{3}\right) \delta 1.38(\mathrm{t}, J=6.9 \mathrm{~Hz}$, 3H), 2.05-2.10 (m, 2H), 2.48-2.57 (m, 4H), 4.36 (q, $J=6.9 \mathrm{~Hz}, 2 \mathrm{H}), 7.39$ (t, $J=4.5 \mathrm{~Hz}$, 1H), 7.54 (dt, $J=8.4,1.8 \mathrm{~Hz}, 2 \mathrm{H}), 7.98(\mathrm{dt}, J=8.7,1.8 \mathrm{~Hz}, 2 \mathrm{H}) ;{ }^{13} \mathrm{C} \mathrm{NMR}\left(\mathrm{CDCl}_{3}\right) \delta$ 14.5, 22.6, 26.8, 38.3, 61.4, 86.8, 91.5, 125.3, 127.7, 129.6, 130.2, 131.9, 155.3, 166.3, 195.6; IR $\left(\mathrm{CH}_{2} \mathrm{Cl}_{2}\right)$ 2981, 1714, $1682 \mathrm{~cm}^{-1}$; HRMS m/z 268.1103 (calcd for $\mathrm{C}_{17} \mathrm{H}_{16} \mathrm{O}_{3}$, 268.1099).

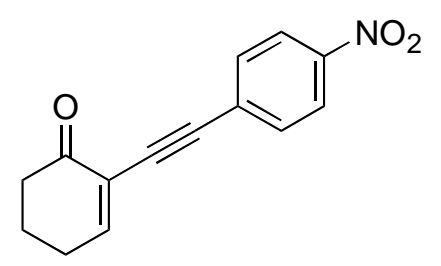

14 
2-(4-Nitrophenyl)ethynyl-2-cyclohexen-1-one (14). This 2-(1-alkynyl)-2-alken-1one was prepared from 2-iodo-2-cyclohexen-1-one ${ }^{2}$ by following the same procedure as that used for compound 8. 2-Iodo-2-cyclohexen-1-one (222 mg, $1.0 \mathrm{mmol})$, (4nitrophenyl)ethyne (294 mg, 2.0 equiv), $\mathrm{PdCl}_{2}\left(\mathrm{PPh}_{3}\right)_{2}$ (35.6 mg, 0.05 equiv), CuI (20 mg, 0.1 equiv) and diisopropylamine ( $0.42 \mathrm{~mL}, 3.0$ equiv) afforded, after purification by flash column chromatography (silica gel, 2:1 hexane/EtOAc), $168 \mathrm{mg}$ (70\%) of the indicated compound 14 as a yellow solid: mp 113-115 ${ }^{\circ} \mathrm{C} ;{ }^{1} \mathrm{H}$ NMR $\left(\mathrm{CDCl}_{3}\right) \delta$ 2.06-2.11 (m, 2H), 2.51-2.58 (m, 4H), 4.36 (q, $J=6.9 \mathrm{~Hz}, 2 \mathrm{H}), 7.44$ (t, $J=4.5 \mathrm{~Hz}, 1 \mathrm{H}), 7.62$ (d, $J=9.0 \mathrm{~Hz}$, 2H), $8.18(\mathrm{~d}, J=9.0 \mathrm{~Hz}, 2 \mathrm{H}) ;{ }^{13} \mathrm{C} \mathrm{NMR}\left(\mathrm{CDCl}_{3}\right) \delta 22.5,26.9,38.3,89.3,90.3,123.8$, 125.0, 130.1, 132.7, 147.3, 156.2, 195.4; IR $\left(\mathrm{CH}_{2} \mathrm{Cl}_{2}\right)$ 3055, 2952, 1682, $1592 \mathrm{~cm}^{-1}$; HRMS m/z 241.0743 (calcd for $\mathrm{C}_{14} \mathrm{H}_{11} \mathrm{NO}_{3}, 241.0739$ ).

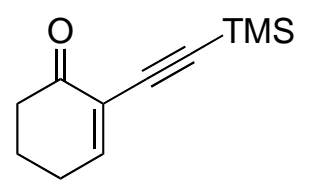

19

2-(Trimethylsilyl)ethynyl-2-cyclohexen-1-one (19). This 2-(1-alkynyl)-2-alken-1one was prepared from 2-iodo-2-cyclohexen-1-one ${ }^{2}$ by following the same procedure as that used for compound 8. 2-Iodo-2-cyclohexen-1-one (222 mg, $1.0 \mathrm{mmol}$ ), (trimethylsilyl)ethyne (196 mg, 2.0 equiv), $\mathrm{PdCl}_{2}\left(\mathrm{PPh}_{3}\right)_{2}$ (35.6 mg, 0.05 equiv), $\mathrm{CuI}$ (20 mg, 0.1 equiv) and diisopropylamine ( $0.42 \mathrm{~mL}, 3.0$ equiv) afforded, after purification by flash column chromatography (silica gel, 5:1 hexane/EtOAc), $159 \mathrm{mg}(83 \%)$ of the indicated compound 19 as a white solid: $\mathrm{mp} 100-101{ }^{\circ} \mathrm{C} ;{ }^{1} \mathrm{H}$ NMR $\left(\mathrm{CDCl}_{3}\right) \delta 0.20(\mathrm{t}, J=$ $3.5 \mathrm{~Hz}, 9 \mathrm{H}), 1.98-2.03$ (m, 2H), 2.40-2.49 (m, 4H), 7.32 (t, $J=4.4 \mathrm{~Hz}, 1 \mathrm{H}) ;{ }^{13} \mathrm{C}$ NMR 
$\left(\mathrm{CDCl}_{3}\right) \delta$ 0.0, 22.4, 26.5, 38.1, 97.6, 99.3, 125.4, 155.5, 195.5; IR $\left(\mathrm{CH}_{2} \mathrm{Cl}_{2}\right) 3042,2960$, 1682, $1349 \mathrm{~cm}^{-1}$; HRMS m/z 192.0974 (calcd for $\mathrm{C}_{11} \mathrm{H}_{16} \mathrm{OSi}$, 192.0970).

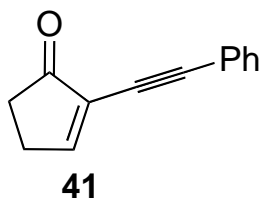

2-Phenylethynyl-2-cyclopenten-1-one (41). This 2-(1-alkynyl)-2-alken-1-one was prepared from 2-iodo-2-cyclopenten-1-one ${ }^{2}$ by following the same procedure as that used for compound 8. 2-Iodo-2-cyclopenten-1-one (208 mg, $1.0 \mathrm{mmol}$ ), phenylacetylene (204 mg, 2.0 equiv), $\mathrm{PdCl}_{2}\left(\mathrm{PPh}_{3}\right)_{2}$ (35.6 mg, 0.05 equiv), $\mathrm{CuI}$ (20 mg, 0.1 equiv) and diisopropylamine ( $0.42 \mathrm{~mL}, 3.0$ equiv) afforded, after purification by flash column chromatography (silica gel, 3:1 hexane/EtOAc), $182 \mathrm{mg}$ (100\%) of the indicated compound 41 as a brown solid: mp 65-66 ${ }^{\circ} \mathrm{C} ;{ }^{1} \mathrm{H}$ NMR $\left(\mathrm{CDCl}_{3}\right) \delta$ 2.49-2.53 (m, 2H), 2.74-2.78 (m, 2H), 7.31-7.33 (m, 3H), 7.49-7.53 (m, 2H), $7.84(\mathrm{t}, J=3.2 \mathrm{~Hz}, 1 \mathrm{H}) ;{ }^{13} \mathrm{C}$ NMR $\left(\mathrm{CDCl}_{3}\right) \delta$ 27.7, 34.3, 80.1, 96.0, 122.7, 128.5, 129.0, 130.3, 132.1, 165.4, 205.9; IR $\left(\mathrm{CH}_{2} \mathrm{Cl}_{2}\right)$ 3061, 2923, 1716, $1488 \mathrm{~cm}^{-1}$; HRMS m/z 182.0734 (calcd for $\mathrm{C}_{13} \mathrm{H}_{10} \mathrm{O}$, 182.0732).

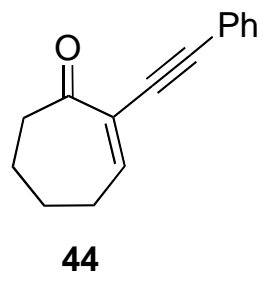

2-Phenylethynyl-2-cyclohepten-1-one (44). This 2-(1-alkynyl)-2-alken-1-one was prepared from 2-iodo-2-cyclohepten-1-one ${ }^{4}$ by following the same procedure as that used for compound 8. 2-Iodo-2-cyclohepten-1-one (236 mg, $1.0 \mathrm{mmol}$ ), phenylacetylene (204 
mg, 2.0 equiv), $\mathrm{PdCl}_{2}\left(\mathrm{PPh}_{3}\right)_{2}$ (35.6 mg, 0.05 equiv), $\mathrm{CuI}$ (20 mg, 0.1 equiv) and diisopropylamine ( $0.42 \mathrm{~mL}$, 3.0 equiv) afforded, after purification by flash column chromatography (silica gel, 5:1 hexane/EtOAc), $90 \mathrm{mg}$ (43\%) of the indicated compound 44 as a yellow oil: ${ }^{1} \mathrm{H}$ NMR $\left(\mathrm{CDCl}_{3}\right) \delta 1.77-1.86$ (m, 4H), 2.47-2.54 (m, 2H), 2.68 (t, $J=$ $6.5 \mathrm{~Hz}, 2 \mathrm{H}), 7.14(\mathrm{t}, J=6.3 \mathrm{~Hz}, 1 \mathrm{H}), 7.25-7.31(\mathrm{~m}, 3 \mathrm{H}), 7.44-7.48(\mathrm{~m}, 2 \mathrm{H}) ;{ }^{13} \mathrm{C} \mathrm{NMR}$ $\left(\mathrm{CDCl}_{3}\right) \delta$ 21.7, 25.2, 28.7, 42.6, 86.6, 90.4, 123.3, 128.4, 128.5, 128.9, 131.9, 151.2, 201.2; IR $\left(\mathrm{CH}_{2} \mathrm{Cl}_{2}\right)$ 3055, 2940, 1679, $1421 \mathrm{~cm}^{-1}$; HRMS m/z 210.1047 (calcd for $\left.\mathrm{C}_{15} \mathrm{H}_{14} \mathrm{O}, 210.1045\right)$.

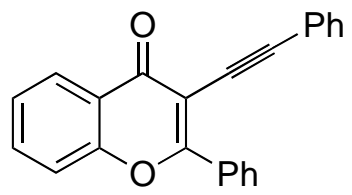

51

2-Phenyl-3-phenylethynyl-4H-benzopyran-4-one (51). This 2-(1-alkynyl)-2-alken1-one was prepared from 3-iodo-2-phenyl-4H-benzopyran-4-one ${ }^{5}$ by the following procedure. To a solution of 3-iodo-2-phenyl-4H-benzopyran-4-one (696 mg, $2.0 \mathrm{mmol}$ ) and phenylacetylene (245 mg, 1.2 equiv) in $\mathrm{Et}_{3} \mathrm{~N}(20 \mathrm{~mL})$ and DMF (1 mL) were added $\mathrm{PdCl}_{2}\left(\mathrm{PPh}_{3}\right)_{2}(14 \mathrm{mg}, 1 \mathrm{~mol} \%)$ and $\mathrm{CuI}(2.0 \mathrm{mg}, 0.5 \mathrm{~mol} \%)$. The resulting mixture was stirred at room temperature overnight. The mixture was diluted with $\mathrm{CHCl}_{3}(100 \mathrm{~mL})$ and washed with $\mathrm{H}_{2} \mathrm{O}(30 \mathrm{~mL})$. The organic layer was dried over $\mathrm{MgSO}_{4}$ and concentrated under reduced pressure. The residue was purified by flash column chromatography (silica gel, 4:1 hexane/EtOAc) to afford $516 \mathrm{mg}(80 \%)$ of the indicated compound 51 as a light yellow solid: $\mathrm{mp} 155-157{ }^{\circ} \mathrm{C} ;{ }^{1} \mathrm{H}$ NMR $\left(\mathrm{CDCl}_{3}\right) \delta$ 7.31-7.35 (m, 3H), 7.42-7.59 (m, 7H), 7.68-7.74 (m, 1H), 8.23-8.32 (m, 3H); IR $\left(\mathrm{CH}_{2} \mathrm{Cl}_{2}\right)$ 3071, 1644, 1614, $1463 \mathrm{~cm}^{-1}$; HRMS m/z 322.0997 (calcd for $\mathrm{C}_{23} \mathrm{H}_{14} \mathrm{O}_{2}$, 322.0994). 

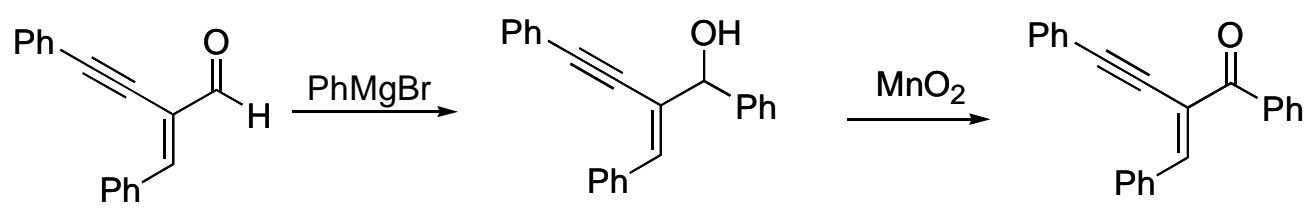

57

(E)-2-Benzylidene-1,4-diphenylbut-3-yn-1-one (57). This 2-(1-alkynyl)-2-alken-1one was prepared from (E)-2-benzylidene-4-phenylbut-3-ynal ${ }^{6}$ by following a procedure from the literature. ${ }^{7}$ To a solution of (E)-2-benzylidene-4-phenylbut-3-ynal (531 mg, 2.3 mmol) in dry THF (5 mL) cooled to $-78{ }^{\circ} \mathrm{C}$ was added $\mathrm{PhMgBr}(1 \mathrm{M}$ in THF, $3.5 \mathrm{~mL}$, $3.5 \mathrm{mmol})$. The reaction was stirred at the same temperature for $30 \mathrm{~min}$ and quenched with satd aq $\mathrm{NH}_{4} \mathrm{Cl}(3 \mathrm{~mL})$. The resulting mixture was extracted with $\mathrm{Et}_{2} \mathrm{O}(20 \mathrm{~mL})$. The organic layer was washed with brine (20 mL), dried over anhydrous $\mathrm{MgSO}_{4}$, filtered and concentrated under reduced pressure. The residue was dissolved in dry THF (30 mL). To this solution was added $\mathrm{MnO}_{2}$ (4.0 g, 20 equiv), followed by stirring at room temperature for $12 \mathrm{~h}$. The reaction mixture was filtered through a short pad of Celite, which was rinsed with EtOAc. The combined organic layer was concentrated under reduced pressure, and the residue was purified by flash column chromatography (silica gel, 7:1 hexane/EtOAc) to afford $470 \mathrm{mg}(66 \%)$ of the indicated compound 57 as a light yellow oil: ${ }^{1} \mathrm{H}$ NMR $\left(\mathrm{CDCl}_{3}\right) \delta$ 7.33-7.38 (m, 3H), 7.41-7.53 (m, 7H), 7.57-7.63 (m, 1H), 7.67 (s, 1H), 8.02-8.06 (m, 2H), 8.13-8.17 (m, 2H); ${ }^{13} \mathrm{C} \mathrm{NMR}\left(\mathrm{CDCl}_{3}\right) \delta 87.5,101.2$, 121.2, 123.1, 128.4, 128.7, 128.9, 129.1, 130.0, 130.7, 130.9, 131.6, 132.8, 135.1, 137.4, 145.4, 193.6; IR $\left(\mathrm{CH}_{2} \mathrm{Cl}_{2}\right)$ 3059, 1663, 1597, 1564, 1490, 1446, 1318, $1264 \mathrm{~cm}^{-1}$; HRMS m/z 308.1209 (calcd for $\mathrm{C}_{23} \mathrm{H}_{16} \mathrm{O}, 308.1201$ ). 
Representative procedure for the $\mathrm{AuCl}_{3}$-catalyzed cyclizations. A solution of $\mathrm{AuCl}_{3}$ (30.3 mg) in MeCN (970 mg) was prepared. To the appropriate 2-(1-alkynyl)-2alken-1-one (0.2 mmol) and nucleophile (1.5 equiv) in $\mathrm{CH}_{2} \mathrm{Cl}_{2}(1.0 \mathrm{~mL})$, was added a portion of the above $\mathrm{AuCl}_{3}$ solution (20 mg, $1 \mathrm{~mol} \%$ ). The mixture was stirred at room temperature for $1 \mathrm{~h}$ unless otherwise specified. The solvent was removed under reduced pressure and the residue was purified by flash chromatography on silica gel.

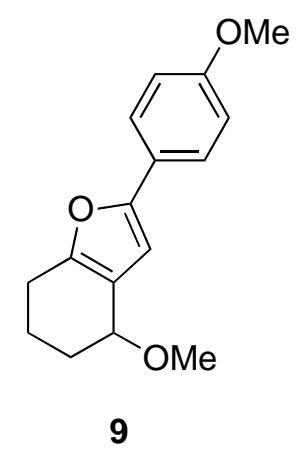

4-Methoxy-2-(4-methoxyphenyl)-4,5,6,7-tetrahydrobenzofuran (9). Compound 8 (45.2 mg, $0.2 \mathrm{mmol}$ ) was allowed to react with methanol (9.6 mg, 1.5 equiv) under our standard reaction conditions for $1 \mathrm{~h}$. The reaction mixture was chromatographed (silica gel, 7:1 hexane/EtOAc) to afford $45.5 \mathrm{mg}$ (88\%) of the indicated compound 9 as a colorless oil: ${ }^{1} \mathrm{H}$ NMR $\left(\mathrm{CDCl}_{3}\right) \delta$ 1.74-1.88 (m, 2H), 1.91-2.11 (m, 2H), 2.53-2.64 (m, 1H), 2.67-2.77 (m, 1H), 3.45 (s, 3H), 3.82 (s, 3H), 4.29 (t, $J=3.9 \mathrm{~Hz}, 1 \mathrm{H}), 6.51$ (s, 1H), 6.90 (dt, $J=9.0,3.0 \mathrm{~Hz}, 2 \mathrm{H}), 7.56(\mathrm{dt}, J=9.0,3.0 \mathrm{~Hz}, 2 \mathrm{H}) ;{ }^{13} \mathrm{C} \mathrm{NMR}\left(\mathrm{CDCl}_{3}\right) \delta 19.2$, 23.5, 28.7, 55.5, 56.4, 72.7, 104.0, 114.3, 120.0, 124.5, 125.1, 152.3, 152.5, 159.0; IR $\left(\mathrm{CH}_{2} \mathrm{Cl}_{2}\right)$ 2943, 1613, 1503, 1463, $1411 \mathrm{~cm}^{-1}$; HRMS m/z 258.1260 (calcd for $\mathrm{C}_{16} \mathrm{H}_{18} \mathrm{O}_{3}$, 258.1256). 


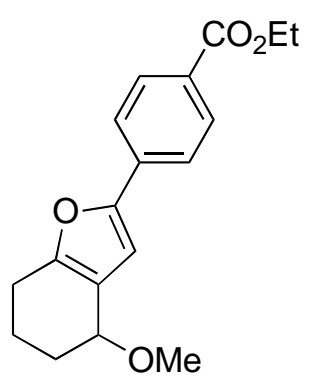

12

\section{Ethyl 4-(4-methoxy-4,5,6,7-tetrahydro-1-benzofuran-2-yl)benzoate (12).}

Compound 11 (53.6 mg, $0.2 \mathrm{mmol}$ ) was allowed to react with methanol (9.6 mg, 1.5 equiv) under our standard reaction conditions for $1 \mathrm{~h}$. The reaction mixture was chromatographed (silica gel, 3:1 hexane/EtOAc) to afford $54.7 \mathrm{mg}$ (91\%) of the indicated compound 12 as a light yellow oil: ${ }^{1} \mathrm{H}$ NMR $\left(\mathrm{CDCl}_{3}\right) \delta 1.39(\mathrm{t}, J=7.4 \mathrm{~Hz}, 3 \mathrm{H}), 1.75-$ 1.88 (m, 2H), 1.91-2.11 (m, 2H), 2.54-2.66 (m, 1H), 2.68-2.78 (m, 1H), 3.44 (s, 3H), 4.29 (t, $J=3.9 \mathrm{~Hz}, 1 \mathrm{H}), 4.37$ (q, $J=6.9 \mathrm{~Hz}, 2 \mathrm{H}), 6.78(\mathrm{~s}, 1 \mathrm{H}), 7.66$ (dd, $J=6.9,1.8 \mathrm{~Hz}, 2 \mathrm{H})$, $8.03(\mathrm{dd}, J=6.9,1.8 \mathrm{~Hz}, 2 \mathrm{H}) ;{ }^{13} \mathrm{C} \mathrm{NMR}\left(\mathrm{CDCl}_{3}\right) \delta 14.6,19.0,23.5,28.5,56.4,61.1$, 72.5, 107.8, 120.7, 123.1, 128.6, 130.3, 135.2, 151.5, 154.4, 166.6; IR $\left(\mathrm{CH}_{2} \mathrm{Cl}_{2}\right)$ 2942, 1713, 1607, 1574, $1423 \mathrm{~cm}^{-1}$; HRMS m/z 300.1365 (calcd for $\mathrm{C}_{18} \mathrm{H}_{20} \mathrm{O}_{4}, 300.1362$ ).

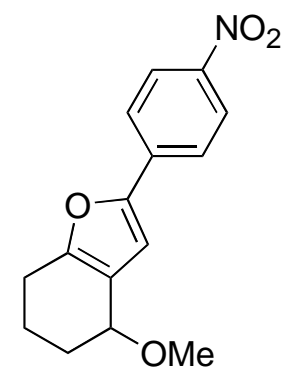

15

4-Methoxy-2-(4-nitrophenyl)-4,5,6,7-tetrahydrobenzofuran (15). Compound 14 (48.3 mg, $0.2 \mathrm{mmol}$ ) was allowed to react with methanol (9.6 mg, 1.5 equiv) under our 
standard reaction conditions for $1 \mathrm{~h}$. The reaction mixture was chromatographed (silica gel, 3:1 hexane/EtOAc) to afford $54.3 \mathrm{mg}$ (99\%) of the indicated compound $\mathbf{1 5}$ as a yellow oil: ${ }^{1} \mathrm{H}$ NMR $\left(\mathrm{CDCl}_{3}\right) \delta$ 1.76-1.89 (m, 2H), 1.91-2.11 (m, 2H), 2.54-2.67 (m, 1H), 2.69-2.79 (m, 1H), 3.44 (s, 3H), 4.30 (t, $J=3.6 \mathrm{~Hz}, 1 \mathrm{H}), 6.87$ (s, $1 \mathrm{H}), 7.70$ (dt, $J=9.0$, $2.4 \mathrm{~Hz}, 2 \mathrm{H}), 8.19$ (dt, $J=9.3,2.1 \mathrm{~Hz}, 2 \mathrm{H}) ;{ }^{13} \mathrm{C} \mathrm{NMR}\left(\mathrm{CDCl}_{3}\right) \delta$ 18.9, 23.5, 28.3, 56.5, 72.4, 109.7, 121.4, 123.6, 124.5, 136.9, 146.2, 150.3, 155.7; IR $\left(\mathrm{CH}_{2} \mathrm{Cl}_{2}\right)$ 2943, 1595, 1514, $1453 \mathrm{~cm}^{-1}$; HRMS m/z 273.1004 (calcd for $\mathrm{C}_{15} \mathrm{H}_{15} \mathrm{NO}_{4}, 273.1001$ ).

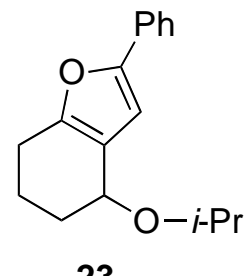

23

4-Isopropoxy-2-phenyl-4,5,6,7-tetrahydrobenzofuran (23). Compound 1 (39.2 mg, $0.2 \mathrm{mmol}$ ) was allowed to react with isopropanol (18 mg, 1.5 equiv) under our standard reaction conditions for $1 \mathrm{~h}$. The reaction mixture was chromatographed (silica gel, 9:1 hexane/EtOAc) to afford $36.1 \mathrm{mg}$ (71\%) of the indicated compound 23 as a colorless oil: ${ }^{1} \mathrm{H}$ NMR $\left(\mathrm{CDCl}_{3}\right) \delta$ 1.22-1.26 (m, 6H), 1.77-1.88 (m, 3H), 2.00-2.13 (m, 1H), 2.53-2.64 (m, 1H), 2.68-2.75 (m, 1H), 3.77-3.89 (m, 1H), 4.46 (t, $J=3.8 \mathrm{~Hz}, 1 \mathrm{H}), 6.60$ (s, 1H),

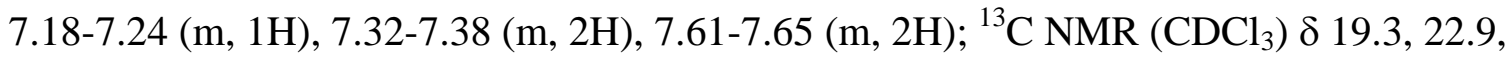
23.4, 23.5, 30.0, 68.7, 69.6, 105.3, 121.2, 123.7, 127.0, 128.8, 131.5, 152.5, 152.9; IR $\left(\mathrm{CH}_{2} \mathrm{Cl}_{2}\right)$ 2968, 1604, 1487, 1449, 1122, $1066 \mathrm{~cm}^{-1}$; HRMS m/z 256.1467 (calcd for $\left.\mathrm{C}_{17} \mathrm{H}_{20} \mathrm{O}_{2}, 256.1463\right)$. 


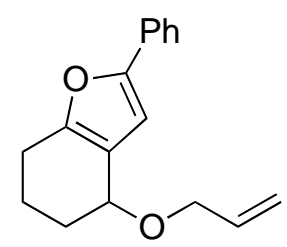

25

4-Allyloxy-2-phenyl-4,5,6,7-tetrahydrobenzofuran (25). Compound 1 (39.2 mg, 0.2 mmol) was allowed to react with allyl alcohol (17.4 mg, 1.5 equiv) under our standard reaction conditions for $1 \mathrm{~h}$. The reaction mixture was chromatographed $\left(\mathrm{Al}_{2} \mathrm{O}_{3}, 70: 1\right.$ hexane/EtOAc) to afford $37.8 \mathrm{mg}$ (75\%) of the indicated compound $\mathbf{2 5}$ as a colorless oil: ${ }^{1} \mathrm{H}$ NMR $\left(\mathrm{CDCl}_{3}\right) \delta$ 1.78-1.88 (m, 2H), 1.92-2.17 (m, 2H), 2.55-2.66 (m, 1H), 2.70-2.79 (m, 1H), 4.12-4.15 (m, 2H), 4.46 (t, $J=3.8 \mathrm{~Hz}, 1 \mathrm{H}), 5.22$ (dd, $J=10.4,1.4 \mathrm{~Hz}, 1 \mathrm{H})$, 5.31-5.39 (m, 1H), 5.93-6.07 (m, 1H), 6.65 (s, 1H), 7.20-7.26 (m, 1H), 7.33-7.39 (m, 2H), 7.62-7.66 (m, 2H); ${ }^{13} \mathrm{C}$ NMR $\left(\mathrm{CDCl}_{3}\right) \delta 19.2,23.5,29.2,69.7,70.7,105.5,116.9$, 120.4, 123.7, 127.1, 128.8, 131.4, 135.7, 152.5, 153.2; IR $\left(\mathrm{CH}_{2} \mathrm{Cl}_{2}\right)$ 3079, 2943, 1633, 1553, $1449 \mathrm{~cm}^{-1}$; HRMS m/z 254.1311 (calcd for $\mathrm{C}_{17} \mathrm{H}_{18} \mathrm{O}_{2}, 254.1307$ ).

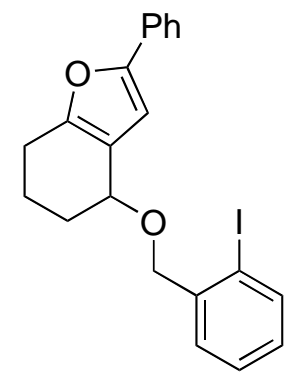

27

4-[(2-Iodobenzyl)oxy]-2-phenyl-4,5,6,7-tetrahydrobenzofuran (27). Compound 1 (39.2 mg, $0.2 \mathrm{mmol}$ ) was allowed to react with 2-iodobenzyl alcohol (70.2 mg, 1.5 equiv) under our standard reaction conditions for $1 \mathrm{~h}$. The reaction mixture was chromatographed $\left(\mathrm{Al}_{2} \mathrm{O}_{3}, 90: 1\right.$ hexane/EtOAc) to afford $75.1 \mathrm{mg}(87 \%)$ of the indicated compound 27 as a light yellow oil: ${ }^{1} \mathrm{H}$ NMR $\left(\mathrm{CDCl}_{3}\right) \delta 1.82-1.95(\mathrm{~m}, 2 \mathrm{H}), 2.04-2.22(\mathrm{~m}$, 
2H), 2.58-2.69 (m, 1H), 2.73-2.80 (m, 1H), 4.59 (t, $J=4.2 \mathrm{~Hz}, 1 \mathrm{H}), 4.64$ (d, $J=3.9 \mathrm{~Hz}$, 2H), 6.71 (s, 1H), 6.98 (dt, $J=7.8,1.7 \mathrm{~Hz}, 1 \mathrm{H}), 7.20-7.25$ (m, 1H), 7.34-7.39 (m, 3H), 7.52 (dd, $J=7.5,1.5 \mathrm{~Hz}, 1 \mathrm{H}), 7.63-7.67(\mathrm{~m}, 2 \mathrm{H}), 7.84(\mathrm{dd}, J=7.8,1.2 \mathrm{~Hz}, 1 \mathrm{H}) ;{ }^{13} \mathrm{C}$ $\operatorname{NMR}\left(\mathrm{CDCl}_{3}\right) \delta$ 19.3, 23.5, 29.1, 71.4, 74.6, 98.3, 105.6, 120.2, 123.7, 127.2, 128.5, 128.8, 129.3, 129.4, 131.4, 139.4, 141.3, 152.6, 153.3; IR $\left(\mathrm{CH}_{2} \mathrm{Cl}_{2}\right)$ 3059, 2942, 1604, 1553, $1486 \mathrm{~cm}^{-1}$; HRMS m/z 430.0437 (calcd for $\mathrm{C}_{21} \mathrm{H}_{19} \mathrm{IO}_{2}, 430.0430$ ).

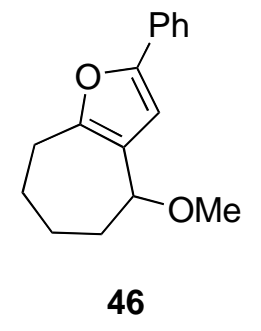

4-Methoxy-2-phenyl-5,6,7,8-tetrahydro-4H-cyclohepta[b]furan (46). Compound 44 (42 mg, $0.2 \mathrm{mmol}$ ) was allowed to react with methanol (9.6 mg, 1.5 equiv) under our standard reaction conditions for $1 \mathrm{~h}$. The reaction mixture was chromatographed (silica gel, 9:1 hexane/EtOAc) to afford $39.7 \mathrm{mg}$ (82\%) of the indicated compound 9 as a light yellow oil: ${ }^{1} \mathrm{H}$ NMR $\left(\mathrm{CDCl}_{3}\right) \delta$ 1.70-1.90 (m, 4H), 1.99-2.14 (m, 2H), 2.84-2.90 (m, 2H), 3.37 (s, 3H), 4.20 (dd, $J=6.3,2.4 \mathrm{~Hz}, 1 \mathrm{H}), 6.59$ (s, 1H), 7.17-7.24 (m, 1H), 7.32-7.38 (m, 2H), 7.59-7.63 (m, 2H); ${ }^{13} \mathrm{C} \mathrm{NMR}\left(\mathrm{CDCl}_{3}\right) \delta 24.4,26.6,28.9,33.3,56.6,75.6,108.4$, 123.48, 123.54, 126.9, 128.8, 131.3, 150.3, 154.0; IR $\left(\mathrm{CH}_{2} \mathrm{Cl}_{2}\right)$ 2927, 2845, 1602, 1552, 1486, 1447, $1084 \mathrm{~cm}^{-1}$; HRMS m/z 242.1310 (calcd for $\mathrm{C}_{16} \mathrm{H}_{18} \mathrm{O}_{2}, 242.1307$ ).

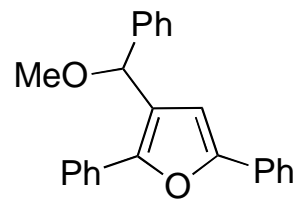

59 
3-[Methoxy(phenyl)methyl]-2,5-diphenylfuran (59). Compound 57 (61.6 mg, 0.2 mmol) was allowed to react with methanol (9.6 mg, 1.5 equiv) under our standard reaction conditions for $4 \mathrm{~h}$ ( $2 \mathrm{~mol} \%$ of $\mathrm{AuCl}_{3}$ was used). The reaction mixture was chromatographed (silica gel, 12:1 hexane/EtOAc) to afford $60.4 \mathrm{mg}$ (89\%) of the indicated compound 59 as a colorless oil: ${ }^{1} \mathrm{H}$ NMR $\left(\mathrm{CDCl}_{3}\right) \delta 3.44(\mathrm{~s}, 3 \mathrm{H}), 5.57(\mathrm{~s}, 1 \mathrm{H})$, 6.75 (s, 1H), 7.25-7.54 (m, 11H), 7.72-7.76 (m, 4H); ${ }^{13} \mathrm{C} \mathrm{NMR}\left(\mathrm{CDCl}_{3}\right)$ \& 57.0, 77.9, 107.3, 124.1, 124.2, 126.8, 127.4, 127.8, 128.1, 128.8, 128.9, 129.0, 130.8, 131.1, 141.1, 150.5, 153.1 (one $\mathrm{sp}^{2}$ carbon missing due to overlap); $\mathrm{IR}\left(\mathrm{CH}_{2} \mathrm{Cl}_{2}\right)$ 3060, 2933, 1594, 1493, $1086 \mathrm{~cm}^{-1}$; HRMS m/z 340.1469 (calcd for $\mathrm{C}_{24} \mathrm{H}_{20} \mathrm{O}_{2}$, 340.1463).

Representative procedure for the iodine-induced cyclizations. To the mixture of appropriate 2-(1-alkynyl)-2-alken-1-one (0.2 mmol), $\mathrm{I}_{2}$ (3.0 equiv) and $\mathrm{NaHCO}_{3}$ (3.0 equiv), was added a solution of nucleophile (8.0 equiv) in $\mathrm{CH}_{3} \mathrm{CN}(2.0 \mathrm{~mL})$. The resulting mixture was stirred at room temperature for $1 \mathrm{~h}$ unless otherwise specified. The mixture was diluted with ether $(25 \mathrm{~mL})$, washed with satd $\mathrm{Na}_{2} \mathrm{~S}_{2} \mathrm{O}_{3}(15 \mathrm{~mL})$ and dried $\left(\mathrm{MgSO}_{4}\right)$. The solvent was removed under reduced pressure and the residue was purified by flash chromatography on silica gel.

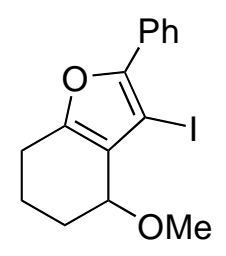

3

3-Iodo-4-methoxy-2-phenyl-4,5,6,7-tetrahydrobenzofuran (3). Compound 1 (39.2 $\mathrm{mg}, 0.2 \mathrm{mmol}$ ) was allowed to react with methanol (51.2 mg, 8.0 equiv) under our standard reaction conditions for $1 \mathrm{~h}$. The reaction mixture was chromatographed (silica gel, 10:1 hexane/EtOAc) to afford $56.8 \mathrm{mg}(80 \%)$ of the indicated compound 3 as a 
colorless oil: ${ }^{1} \mathrm{H}$ NMR $\left(\mathrm{CDCl}_{3}\right) \delta$ 1.52-1.65 (m, 1H), 1.83-2.10 (m, 2H), 2.14-2.23 (m, 1H), 2.51-2.63 (m, 1H), 2.69-2.79 (m, 1H), 3.52 (s, 3H), 4.13 (t, $J=2.9 \mathrm{~Hz}, 1 \mathrm{H}), 7.27-$ 7.34 (m, 1H), 7.37-7.44 (m, 2H), 7.90-7.98 (m, 2H); ${ }^{13} \mathrm{C} \mathrm{NMR}\left(\mathrm{CDCl}_{3}\right) \delta$ 18.5, 23.5, 27.1, 57.3, 65.3, 72.2, 123.7, 126.4, 128.1, 128.5, 130.8, 150.4, 154.1; IR $\left(\mathrm{CH}_{2} \mathrm{Cl}_{2}\right) 2938$, 2817, 1628, 1483, 1414, $1083 \mathrm{~cm}^{-1}$; HRMS m/z 354.0121 (calcd for $\mathrm{C}_{15} \mathrm{H}_{15} \mathrm{IO}_{2}$, 354.0117).

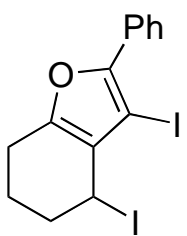

7

3,4-Diiodo-2-phenyl-4,5,6,7-tetrahydrobenzofuran (7). Compound 1 (39.2 mg, 0.2 mmol) was allowed to react under our standard reaction conditions, in the absence of any nucleophile for $1 \mathrm{~h}$. The reaction mixture was chromatographed (silica gel, 40:1 hexane/EtOAc) to afford $55.0 \mathrm{mg}$ (41\%) of the indicated compound 7 as a white solid: mp 160-162 ${ }^{\circ} \mathrm{C} ;{ }^{1} \mathrm{H}$ NMR $\left(\mathrm{CDCl}_{3}\right) \delta$ 1.49-1.60 (m, 1H), 1.86-2.00 (m, 1H), 2.38-2.66 (m, 2H), 2.73-2.84 (m, 2H), 4.56 (s, 1H), 7.26-7.33 (m, 1H), 7.37-7.43 (m, 2H), 7.86-7.90 (m, 2H); ${ }^{13} \mathrm{C} \mathrm{NMR}\left(\mathrm{CDCl}_{3}\right) \delta$ 19.1, 23.5, 27.3, 65.3, 67.9, 123.0, 126.7, 128.1, 128.5, 130.9, 150.4, 155.1; IR $\left(\mathrm{CH}_{2} \mathrm{Cl}_{2}\right)$ 3060, 2984, 2941, 1620, 1603, $1483 \mathrm{~cm}^{-1}$.

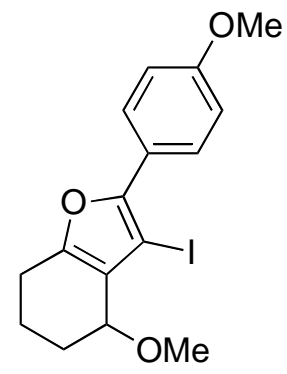




\section{3-Iodo-4-methoxy-2-(4-methoxyphenyl)-4,5,6,7-tetrahydrobenzofuran (10).}

Compound 8 (45.2 mg, 0.2 mmol) was allowed to react with methanol (51.2 mg, 8.0 equiv) under our standard reaction conditions for $1 \mathrm{~h}$. The reaction mixture was chromatographed (silica gel, 6:1 hexane/EtOAc) to afford $63.9 \mathrm{mg}$ (83\%) of the indicated compound 10 as a colorless oil: ${ }^{1} \mathrm{H} \mathrm{NMR}\left(\mathrm{CDCl}_{3}\right) \delta 1.58(\mathrm{tt}, J=3.5,13.6 \mathrm{~Hz}, 1 \mathrm{H}), 1.81$ 1.92 (m, 1H), 1.94-2.10 (m, 1H), 2.12-2.21 (m, 1H), 2.50-2.61 (m, 1H), 2.67-2.76 (m, 1H), 3.51 (s, 3H), 3.83 (s, 3H), 4.11 (t, $J=3.0 \mathrm{~Hz}, 1 \mathrm{H}), 6.94$ (dt, $J=9.0,2.7 \mathrm{~Hz}, 2 \mathrm{H}$ ), $7.85(\mathrm{dt}, J=9.0,2.7 \mathrm{~Hz}, 2 \mathrm{H}) ;{ }^{13} \mathrm{C} \mathrm{NMR}\left(\mathrm{CDCl}_{3}\right) \delta 18.5,23.5,27.1,55.5,57.3,63.8$, 72.3, 114.0, 123.4, 123.6, 128.0, 150.6, 153.5, 159.5; IR $\left(\mathrm{CH}_{2} \mathrm{Cl}_{2}\right)$ 2939, 2835, 1612, $1495 \mathrm{~cm}^{-1}$; HRMS m/z 384.0229 (calcd for $\mathrm{C}_{16} \mathrm{H}_{17} \mathrm{IO}_{3}, 384.0223$ ).

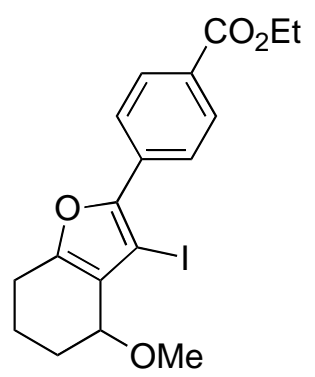

13

\section{Ethyl 4-(3-iodo-4-methoxy-4,5,6,7-tetrahydrobenzofuran-2-yl)benzoate (13).}

Compound 11 (53.6 mg, $0.2 \mathrm{mmol}$ ) was allowed to react with methanol (51.2 mg, 8.0 equiv) under our standard reaction conditions for $1 \mathrm{~h}$. The reaction mixture was chromatographed (silica gel, 5:1 hexane/EtOAc) to afford $70.7 \mathrm{mg}$ (83\%) of the indicated compound 13 as a colorless oil: ${ }^{1} \mathrm{H}$ NMR $\left(\mathrm{CDCl}_{3}\right) \delta 1.40(\mathrm{t}, J=7.2 \mathrm{~Hz}, 3 \mathrm{H}), 1.62(\mathrm{tt}, J=$ 3.3, $13.8 \mathrm{~Hz}, 1 \mathrm{H}), 1.88-2.10$ (m, 2H), 2.14-2.22 (m, 1H), 2.50-2.62 (m, 1H), 2.68-2.79 (m, 1H), 3.51 (s, 3H), 4.12 (t, $J=3.0 \mathrm{~Hz}, 1 \mathrm{H}), 4.38$ (q, $J=7.2 \mathrm{~Hz}, 3 \mathrm{H}), 8.02-8.09$ (m, $4 \mathrm{H}) ;{ }^{13} \mathrm{C} \mathrm{NMR}\left(\mathrm{CDCl}_{3}\right) \delta 14.6,18.4,23.5,26.9,57.3,61.2,67.6,72.1,124.4,125,7$, 
129.4, 129.9, 134.7, 149.2, 155.1, 166.5; IR $\left(\mathrm{CH}_{2} \mathrm{Cl}_{2}\right)$ 2932, 2817, 1713, $1607,1276 \mathrm{~cm}^{-1}$; HRMS m/z 426.0336 (calcd for $\mathrm{C}_{18} \mathrm{H}_{19} \mathrm{IO}_{4}, 426.0328$ ).

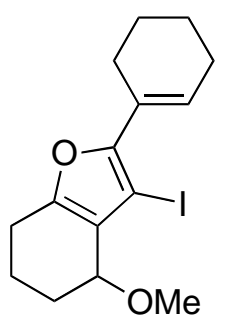

18

\section{2-(Cyclohex-1-enyl)-3-iodo-4-methoxy-4,5,6,7-tetrahydrobenzofuran (18).}

Compound 16 (40.0 mg, $0.2 \mathrm{mmol}$ ) was allowed to react with methanol (51.2 mg, 8.0 equiv) under our standard reaction conditions for $1 \mathrm{~h}$. The reaction mixture was chromatographed (silica gel, 11:1 hexane/EtOAc) to afford $33.0 \mathrm{mg}$ (46\%) of the indicated compound 18 as a colorless oil: ${ }^{1} \mathrm{H}$ NMR $\left(\mathrm{CDCl}_{3}\right) \delta 1.43-1.75(\mathrm{~m}, 5 \mathrm{H}), 1.77-$ 1.85 (m, 1H), 1.92-2.06 (m, 1H), 2.08-2.22 (m, 3H), 2.40-2.52 (m, 3H), 2.58-2.66 (m, 1H), 3.47 (s, 3H), 4.04 (s, 1H), 6.42-6.46 (m, 1H); ${ }^{13} \mathrm{C} \mathrm{NMR}\left(\mathrm{CDCl}_{3}\right) \delta$ 18.5, 22.1, 22.8, 23.4, 25.6, 26.7, 27.1, 57.2, 63.0, 72.3, 122.8, 127.9, 128.2, 152.4, 152.5; IR $\left(\mathrm{CH}_{2} \mathrm{Cl}_{2}\right)$ 2928, 2858, 1629, 1435, $1346 \mathrm{~cm}^{-1}$; HRMS m/z 358.0435 (calcd for $\mathrm{C}_{15} \mathrm{H}_{19} \mathrm{IO}_{2}$, 358.0430).

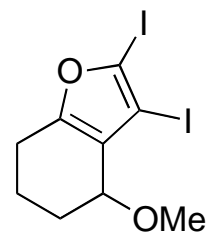

21

2,3-Diiodo-4-methoxy-4,5,6,7-tetrahydrobenzofuran (21). Compound 19 (38.4 mg, $0.2 \mathrm{mmol}$ ) was allowed to react with methanol (51.2 $\mathrm{mg}$, 8.0 equiv) under our standard reaction conditions for $1 \mathrm{~h}$. The reaction mixture was chromatographed (silica gel, 9:1 
hexane/EtOAc) to afford $47.2 \mathrm{mg}(60 \%)$ of the indicated compound 21 as a colorless oil: ${ }^{1} \mathrm{H}$ NMR $\left(\mathrm{CDCl}_{3}\right) \delta$ 1.47-1.60 (m, 1H), 1.72-1.84 (m, 1H), 1.89-2.13 (m, 2H), 2.48-2.59 (m, 1H), 2.64-2.73 (m, 1H), 3.46 (s, 3H), $4.04(\mathrm{t}, J=3.0 \mathrm{~Hz}, 1 \mathrm{H}),{ }^{13} \mathrm{C} \mathrm{NMR}\left(\mathrm{CDCl}_{3}\right) \delta$ 18.3, 23.7, 27.2, 57.4, 72.0, 83.1, 96.4, 124.2, 159.8; IR $\left(\mathrm{CH}_{2} \mathrm{Cl}_{2}\right)$ 2940, 2817, 1625, 1452, $1410 \mathrm{~cm}^{-1}$; HRMS m/z 403.8777 (calcd for $\mathrm{C}_{9} \mathrm{H}_{10} \mathrm{I}_{2} \mathrm{O}_{2}, 403.8770$ ).

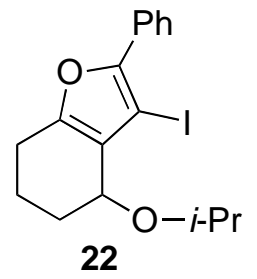

3-Iodo-4-isopropoxy-2-phenyl-4,5,6,7-tetrahydrobenzofuran (22). Compound 1 (39.2 mg, $0.2 \mathrm{mmol}$ ) was allowed to react with isopropanol (96 mg, 8.0 equiv) under our standard reaction conditions for $1 \mathrm{~h}$. The reaction mixture was chromatographed (silica gel, 12:1 hexane/EtOAc) to afford $55.5 \mathrm{mg}$ (73\%) of the indicated compound 22 as a light yellow oil: ${ }^{1} \mathrm{H}$ NMR $\left(\mathrm{CDCl}_{3}\right) \delta 1.25(\mathrm{~d}, J=6.3 \mathrm{~Hz}, 3 \mathrm{H}), 1.36(\mathrm{~d}, J=6.0 \mathrm{~Hz}, 3 \mathrm{H})$, 1.53-1.63 (m, 1H), 1.82-1.93 (m, 1H), 2.02-2.17 (m, 2H), 2.50-2.62 (m, 1H), 2.69-2.78 (m, 1H), 3.87-3.97 (m, 1H), 4.35 (s, 3H), 7.26-7.33 (m, 1H), 7.37-7.43 (m, 2H), 7.907.94 (m, 2H); ${ }^{13} \mathrm{C} \mathrm{NMR}\left(\mathrm{CDCl}_{3}\right) \delta 18.3,23.2,23.6,24.2,28.6,65.3,68.7,70.5,123.7$, 126.6, 128.0, 128.5, 130.9, 150.3, 154.2; IR $\left(\mathrm{CH}_{2} \mathrm{Cl}_{2}\right) 3058,2967,2882,1625,1483$, $1445 \mathrm{~cm}^{-1}$; HRMS m/z 382.0437 (calcd for $\mathrm{C}_{17} \mathrm{H}_{19} \mathrm{IO}_{2}, 382.0430$ ).

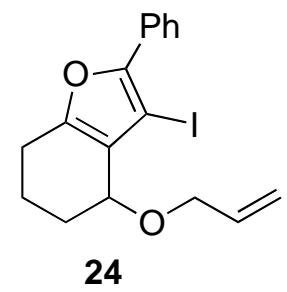


4-Allyoxy-3-iodo-2-phenyl-4,5,6,7-tetrahydrobenzofuran (24). Compound 1 (39.2 mg, $0.2 \mathrm{mmol}$ ) was allowed to react with allyl alcohol (139 mg, 8.0 equiv) under our standard reaction conditions for $1 \mathrm{~h}$. The reaction mixture was chromatographed $\left(\mathrm{Al}_{2} \mathrm{O}_{3}\right.$, 100:1 hexane/EtOAc) to afford $43.3 \mathrm{mg}$ (57\%) of the indicated compound 24 as a light yellow oil: ${ }^{1} \mathrm{H}$ NMR $\left(\mathrm{CDCl}_{3}\right) \delta 1.61(\mathrm{tt}, J=13.5,3.2 \mathrm{~Hz}, 1 \mathrm{H}), 1.83-1.93(\mathrm{~m}, 1 \mathrm{H}), 1.99$ 2.21 (m, 2H), 2.51-2.63 (m, 1H), 2.70-2.79 (m, 1H), 4.15-4.26 (m, 2H), 4.29-4.32 (m, 1H), 5.20 (dd, $J=10.4,1.2 \mathrm{~Hz}, 1 \mathrm{H}), 5.35$ (dd, $J=17.4,1.4 \mathrm{~Hz}, 1 \mathrm{H}), 6.01-6.13$ (m, 1H), 7.28-7.33 (m, 1H), 7.38-7.44 (m, 2H), 7.91-7.96 (m, 2H); ${ }^{13} \mathrm{C} \mathrm{NMR}\left(\mathrm{CDCl}_{3}\right) \delta$ 18.5, 23.5, 27.8, 65.3, 70.7, 70.9, 117.2, 123.7, 126.5, 128.0, 128.5, 130.8, 135.6, 150.3, 154.2; IR $\left(\mathrm{CH}_{2} \mathrm{Cl}_{2}\right)$ 3079, 2940, 2860, 1627, 1603, $1483 \mathrm{~cm}^{-1}$; HRMS m/z 380.0278 (calcd for $\left.\mathrm{C}_{17} \mathrm{H}_{17} \mathrm{IO}_{2}, 380.0273\right)$.

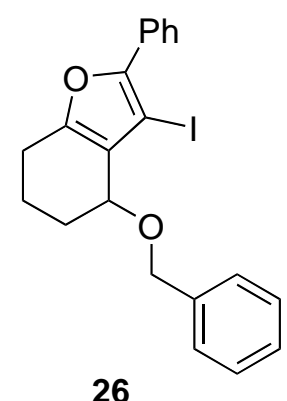

\section{4-Benzyloxy-3-iodo-2-phenyl-4,5,6,7-tetrahydrobenzofuran (26). Compound 1} (39.2 mg, $0.2 \mathrm{mmol}$ ) was allowed to react with benzyl alcohol (173 mg, 8.0 equiv) under our standard reaction conditions for $1 \mathrm{~h}$. The reaction mixture was chromatographed $\left(\mathrm{Al}_{2} \mathrm{O}_{3}, 100: 1\right.$ hexane/EtOAc) to afford $51.5 \mathrm{mg}$ (60\%) of the indicated compound 26 as a light yellow oil: ${ }^{1} \mathrm{H}$ NMR $\left(\mathrm{CDCl}_{3}\right) \delta 1.65(\mathrm{tt}, J=13.5,3.3 \mathrm{~Hz}, 1 \mathrm{H}), 1.85-1.97(\mathrm{~m}, 1 \mathrm{H})$, 2.05-2.20 (m, 1H), 2.21-2.29 (m, 1H), 2.53-2.65 (m, 1H), 2.71-2.80 (m, 1H), 4.43 (t, $J=$ $2.9 \mathrm{~Hz}, 1 \mathrm{H}), 4.73(\mathrm{~s}, 1 \mathrm{H}), 7.25-7.50(\mathrm{~m}, 8 \mathrm{H}), 7.93-7.97(\mathrm{~m}, 2 \mathrm{H}) ;{ }^{13} \mathrm{C} \mathrm{NMR}\left(\mathrm{CDCl}_{3}\right) \delta$ 18.6, 23.5, 27.7, 65.4, 71.1, 72.1, 123.8, 126.5, 127.9, 128.1, 128.5, 128.56, 128.58, 
130.8, 138.8, 150.3, 154.3; IR $\left(\mathrm{CH}_{2} \mathrm{Cl}_{2}\right)$ 3062, 3029, 2940, 1628, 1603, $1484 \mathrm{~cm}^{-1}$;

HRMS m/z 419.9653 (calcd for $\mathrm{C}_{21} \mathrm{H}_{19} \mathrm{IO}_{2}, 419.9647$ ).

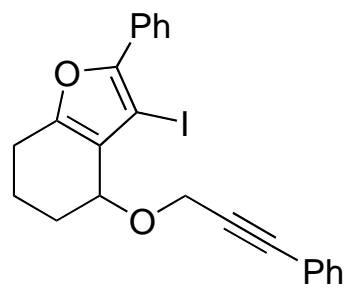

28

\section{3-Iodo-2-phenyl-4-(3-phenyl-2-propynyl)oxy-4,5,6,7-tetrahydrobenzofuran (28).}

Compound 1 (39.2 mg, $0.2 \mathrm{mmol}$ ) was allowed to react with 3-phenyl-2-propyn-1-ol (211 mg, 8.0 equiv) under our standard reaction conditions for $1 \mathrm{~h}$. The reaction mixture was chromatographed (silica gel, 18:1 hexane/EtOAc) to afford $70.0 \mathrm{mg}$ (77\%) of the indicated compound 28 as a colorless oil: ${ }^{1} \mathrm{H}$ NMR $\left(\mathrm{CDCl}_{3}\right) \delta 1.67(\mathrm{tt}, J=13.8,3.3 \mathrm{~Hz}$, 1H), 1.86-1.97 (m, 1H), 2.05-2.20 (m, 1H), 2.28-2.37 (m, 1H), 2.53-2.66 (m, 1H), 2.72$2.82(\mathrm{~m}, 1 \mathrm{H}), 4.56(\mathrm{t}, J=3.2 \mathrm{~Hz}, 1 \mathrm{H}), 4.61(\mathrm{~d}, J=5.7 \mathrm{~Hz}, 2 \mathrm{H}), 7.29-7.36(\mathrm{~m}, 4 \mathrm{H}), 7.38-$ 7.45 (m, 2H), 7.47-7.52 (m, 2H), 7.93-7.98 (m, 2H); ${ }^{13} \mathrm{C} \mathrm{NMR}\left(\mathrm{CDCl}_{3}\right) \delta$ 18.5, 23.5, 27.8, 57.7, 65.1, 70.3, 86.3, 86.4, 123.2, 123.5, 126.5, 128.1, 128.55, 128.58, 128.60, 130.8, 132.0, 150.5, 154.6; IR $\left(\mathrm{CH}_{2} \mathrm{Cl}_{2}\right)$ 3057, 2940, 1660, 1626, $1487 \mathrm{~cm}^{-1}$; HRMS m/z 454.0437 (calcd for $\mathrm{C}_{23} \mathrm{H}_{19} \mathrm{IO}_{2}, 454.0430$ ).

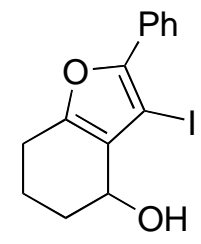

31

3-Iodo-2-phenyl-4,5,6,7-tetrahydrobenzofuran-4-ol (31). Compound 1 (39.2 mg, $0.2 \mathrm{mmol}$ ) was allowed to react with $\mathrm{H}_{2} \mathrm{O}$ (28.8 $\mathrm{mg}$, 8.0 equiv) under our standard reaction conditions for $1 \mathrm{~h}$. The reaction mixture was chromatographed (silica gel, 4:1 
hexane/EtOAc) to afford $52.1 \mathrm{mg}$ (77\%) of the indicated compound 31 as a white solid: mp 83-84 ${ }^{\circ} \mathrm{C} ;{ }^{1} \mathrm{H}$ NMR $\left(\mathrm{CDCl}_{3}\right) \delta$ 1.75-1.96 (m, 3H), 1.99-2.14 (m, 2H), 2.53-2.65 (m, 1H), 2.70-2.79 (m, 1H), 4.70 (q, $J=3.9 \mathrm{~Hz}, 1 \mathrm{H}), 7.29-7.35$ (m, 1H), 7.38-7.45 (m, 2H), 7.92-7.97 (m, 2H); ${ }^{13} \mathrm{C}$ NMR $\left(\mathrm{CDCl}_{3}\right) \delta$ 18.6, 23.5, 31.8, 63.7, 64.1, 125.0, 126.3, 128.2, 128.6, 130.6, 150.3, 153.8; IR $\left(\mathrm{CH}_{2} \mathrm{Cl}_{2}\right) 3340,2940,1624,1483,1442,1223 \mathrm{~cm}^{-1}$; HRMS m/z 339.9970 (calcd for $\mathrm{C}_{14} \mathrm{H}_{13} \mathrm{IO}_{2}$, 339.9960).

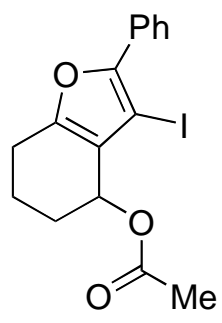

33

3-Iodo-2-phenyl-4,5,6,7-tetrahydrobenzofuran-4-yl acetate (33). Compound 1 (39.2 mg, $0.2 \mathrm{mmol}$ ) was allowed to react with acetic acid (144 mg, 8.0 equiv) under our standard reaction conditions for $1 \mathrm{~h}$. The reaction mixture was chromatographed (silica gel, 6:1 hexane/EtOAc) to afford $50.3 \mathrm{mg}$ (66\%) of the indicated compound 33 as a light yellow solid: mp $115-116{ }^{\circ} \mathrm{C} ;{ }^{1} \mathrm{H}$ NMR $\left(\mathrm{CDCl}_{3}\right) \delta$ 1.82-1.97 (m, 3H), 2.00-2.14 (m, 4H), 2.57-2.65 (m, 1H), 2.76-2.87 (m, 1H), $5.79(\mathrm{t}, J=3.0 \mathrm{~Hz}, 1 \mathrm{H}), 7.29-7.35$ (m, 1H), 7.387.45 (m, 2H), 7.92-7.96 (m, 2H); ${ }^{13} \mathrm{C} \mathrm{NMR}\left(\mathrm{CDCl}_{3}\right) \delta$ 19.1, 21.5, 23.3, 29.3, 63.9, 66.3, 121.6, 126.4, 128.3, 128.6, 130.5, 150.7, 155.3, 170.8; IR $\left(\mathrm{CH}_{2} \mathrm{Cl}_{2}\right)$ 2951, 1731, 1628 , 1483, $1370 \mathrm{~cm}^{-1}$; HRMS m/z 382.0071 (calcd for $\mathrm{C}_{16} \mathrm{H}_{15} \mathrm{IO}_{3}, 382.0066$ ).

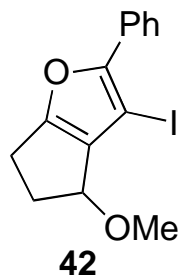


3-Iodo-4-methoxy-2-phenyl-5,6-dihydro-4H-cyclopentafuran (42). Compound 41 (36.4 mg, $0.2 \mathrm{mmol}$ ) was allowed to react with $\mathrm{MeOH}$ (51.2 mg, 8.0 equiv) under our standard reaction conditions for $4 \mathrm{~h}$. The reaction mixture was chromatographed (silica gel, 6:1 hexane/EtOAc) to afford $50.3 \mathrm{mg}$ (74\%) of the indicated compound 42 as a light yellow solid: $\mathrm{mp} 70-7{ }^{\circ} \mathrm{C} ;{ }^{1} \mathrm{H} \mathrm{NMR}\left(\mathrm{CDCl}_{3}\right) \delta 2.36-2.45$ (m, $\left.1 \mathrm{H}\right), 2.64-2.78(\mathrm{~m}, 2 \mathrm{H})$, 2.98-3.06 (m, 1H), 3.47 (s, 3H), 4.65-4.69 (m, 1H), 7.29-7.35 (m, 1H), 7.39-7.45 (m, 2H), 7.92-7.96 (m, 2H); ${ }^{13} \mathrm{C} \mathrm{NMR}\left(\mathrm{CDCl}_{3}\right) \delta$ 24.2, 35.2, 56.6, 60.0, 77.7, 126.5, 128.2, 128.6, 131.1, 133.2, 155.7, 162.3; IR $\left(\mathrm{CH}_{2} \mathrm{Cl}_{2}\right)$ 2974, 2931, 2819, 1619, 1601, $1479 \mathrm{~cm}^{-1}$; HRMS m/z 399.9968 (calcd for $\mathrm{C}_{14} \mathrm{H}_{13} \mathrm{IO}_{2}, 399.9960$ ).

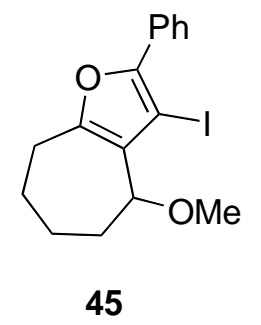

\section{3-Iodo-4-methoxy-2-phenyl-5,6,7,8-tetrahydro-4H-cycloheptafuran (45).}

Compound 44 (42.0 mg, $0.2 \mathrm{mmol}$ ) was allowed to react with $\mathrm{MeOH}$ (51.2 mg, 8.0 equiv) under our standard reaction conditions for $1 \mathrm{~h}$. The reaction mixture was chromatographed (silica gel, 9:1 hexane/EtOAc) to afford $56.0 \mathrm{mg}$ (76\%) of the indicated compound 45 as a light yellow oil: ${ }^{1} \mathrm{H}$ NMR $\left(\mathrm{CDCl}_{3}\right) \delta 1.50-1.68(\mathrm{~m}, 2 \mathrm{H}), 1.78-1.87(\mathrm{~m}$, 1H), 1.96-2.11 (m, 2H), 2.35-2.42 (m, 1H), 2.87-2.94 (m, 2H), 3.40 (s, 3H), 4.28 (dd, $J=$ 1.8, $5.1 \mathrm{~Hz}, 1 \mathrm{H}), 7.25-7.34$ (m, 1H), 7.38-7.44 (m, 2H), 7.91-7.96 (m, 2H); ${ }^{13} \mathrm{C}$ NMR $\left(\mathrm{CDCl}_{3}\right) \delta 23.2,26.4,28.8,31.8,56.6,70.1,74.5,125.0,126.4,128.0,128.5,130.8$, 
149.0, 155.3; IR $\left(\mathrm{CH}_{2} \mathrm{Cl}_{2}\right)$ 3055, 2926, 1602, 1485, $1445 \mathrm{~cm}^{-1}$; HRMS m/z 368.0279

(calcd for $\mathrm{C}_{16} \mathrm{H}_{17} \mathrm{IO}_{2}$, 368.0273).<smiles>COC1Oc2ccccc2-c2oc(-c3ccccc3)c(I)c21</smiles>

48

3-Iodo-4-methoxy-2-phenyl-4H-furo[3,2-c]chromene (48). Compound 47 (49.2 mg, $0.2 \mathrm{mmol}$ ) was allowed to react with $\mathrm{MeOH}$ (12.8 mg, 2.0 equiv) under our standard reaction conditions for $1 \mathrm{~h}$. The reaction mixture was chromatographed (silica gel, 4:1 hexane/EtOAc) to afford $70.1 \mathrm{mg}$ (87\%) of the indicated compound $\mathbf{4 8}$ as a white solid: mp 74-75 ${ }^{\circ} \mathrm{C} ;{ }^{1} \mathrm{H}$ NMR $\left(\mathrm{CDCl}_{3}\right) \delta 3.64$ (s, 3H), 6.10 (s, 1H), 7.06-7.14 (m, 2H), 7.237.29 (m, 1H), 7.36-7.42 (m, 1H), 7.44-7.51 (m, 2H), 7.63 (dd, $J=7.5,1.4$ Hz, 1H), 8.03$8.08(\mathrm{~m}, 2 \mathrm{H}) ;{ }^{13} \mathrm{C} \mathrm{NMR}\left(\mathrm{CDCl}_{3}\right) \delta 55.8,61.2,98.9,114.6,117.4,119.4,120.2,122.3$, 126.7, 128.77, 128.81, 129.5, 130.1, 147.6, 151.6, 152.0; IR $\left(\mathrm{CH}_{2} \mathrm{Cl}_{2}\right)$ 3059, 2927, 2828, 1642, $1497 \mathrm{~cm}^{-1}$; HRMS m/z 403.9918 (calcd for $\mathrm{C}_{18} \mathrm{H}_{13} \mathrm{IO}_{3}, 403.9910$ ).

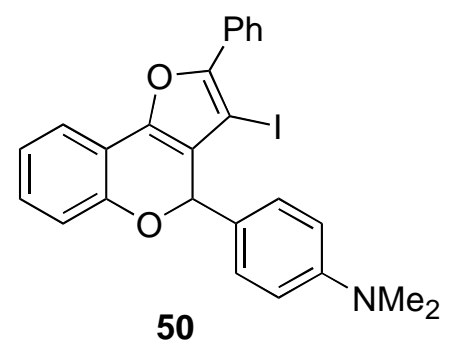

\section{4-[4-(Dimethylamino)phenyl]-3-iodo-2-phenyl-4H-furo[3,2-c]chromene (50).}

Compound 47 (49.2 mg, $0.2 \mathrm{mmol}$ ) was allowed to react with $N, N$-dimethylaniline (36.2 mg, 1.5 equiv) under our standard reaction conditions for $1 \mathrm{~h}$. The reaction mixture was chromatographed (silica gel, 8:1 hexane/EtOAc) to afford $78.4 \mathrm{mg}$ (80\%) of the indicated 
compound 50 as a white solid: mp 157-158 ${ }^{\circ} \mathrm{C} ;{ }^{1} \mathrm{H}$ NMR $\left(\mathrm{CDCl}_{3}\right) \delta 2.94$ (s, 6H), 6.31 (s, 1H), $6.67(\mathrm{~d}, J=8.7 \mathrm{~Hz}, 2 \mathrm{H}), 6.85(\mathrm{~d}, J=8.1 \mathrm{~Hz}, 1 \mathrm{H}), 6.96(\mathrm{t}, J=7.5 \mathrm{~Hz}, 1 \mathrm{H}), 7.09$ (t, $J$ = 7.6 Hz, 1H), 7.27 (d, $J=8.4 \mathrm{~Hz}, 2 \mathrm{H}), 7.34-7.39(\mathrm{~m}, 1 \mathrm{H}), 7.43-7.55(\mathrm{~m}, 3 \mathrm{H}), 8.09$ (d, $J$ $=8.7 \mathrm{~Hz}, 2 \mathrm{H}) ;{ }^{13} \mathrm{C} \mathrm{NMR}\left(\mathrm{CDCl}_{3}\right) \delta 40.6,62.7,79.1,112.3,115.6,117.2,119.8,121.4$, 121.7, 126.5, 127.0, 128.5, 128.7, 129.35, 129.43, 130.3, 146.3, 151.1, 151.6, 152.7 (one $\mathrm{sp}^{2}$ carbon missing due to overlap; IR $\left(\mathrm{CH}_{2} \mathrm{Cl}_{2}\right) 2962,2918,1612,1522 \mathrm{~cm}^{-1}$; HRMS m/z 493.0544 (calcd for $\mathrm{C}_{25} \mathrm{H}_{20} \mathrm{INO}_{2}$, 493.0539).

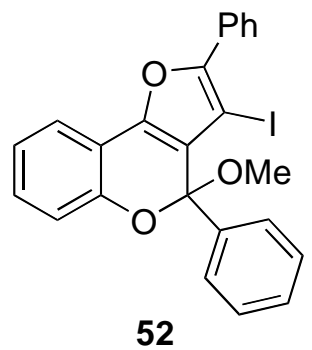

3-Iodo-4-methoxy-2,4-diphenyl-4H-furo[3,2-c]chromene (52). Compound 51 (64.4 mg, $0.2 \mathrm{mmol}$ ) was allowed to react with $\mathrm{MeOH}$ (32.0 mg, 5 equiv) under our standard reaction conditions for $1 \mathrm{~h}$. The reaction mixture was chromatographed (silica gel, 3:1 hexane/EtOAc) to afford $58.7 \mathrm{mg}(61 \%)$ of the indicated compound 52 as a yellow oil: ${ }^{1} \mathrm{H} \mathrm{NMR}\left(\mathrm{CDCl}_{3}\right) \delta 3.39$ (s, 3H), 7.02-7.09 (m, 2H), 7.23-7.28 (m, 1H), 7.36-7.51 (m, 6H), 7.61-7.70 (m, 3H), 8.11 (d, $J=7.5 \mathrm{~Hz}, 2 \mathrm{H}) ;{ }^{13} \mathrm{C} \mathrm{NMR}\left(\mathrm{CDCl}_{3}\right) \delta$ 51.6, 61.7, 105.5, 112.7, 116.1, 117.2, 120.1, 121.5, 127.0, 127.1, 128.2, 128.7, 129.0, 129.05, 129.9, 130.1, 141.4, 148.7, 152.5, 153.1; IR $\left(\mathrm{CH}_{2} \mathrm{Cl}_{2}\right)$ 3061, 3031, 2934, 1636, 1491, $1448 \mathrm{~cm}^{-1}$; HRMS m/z 480.0229 (calcd for $\mathrm{C}_{24} \mathrm{H}_{17} \mathrm{IO}_{3}, 480.0223$ ). 


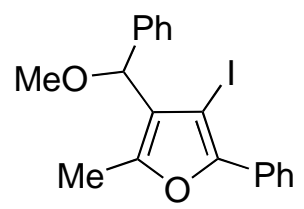

55

3-Iodo-4-[methoxy(phenyl)methyl]-5-methyl-2-phenylfuran (55). Compound 54 (49.2 mg, $0.2 \mathrm{mmol}$ ) was allowed to react with $\mathrm{MeOH}$ (51.2 mg, 8.0 equiv) under our standard reaction conditions for $50 \mathrm{~h}$. The reaction mixture was chromatographed (silica gel, 12:1 hexane/EtOAc) to afford $58.2 \mathrm{mg}$ (72\%) of the indicated compound 55 as a colorless oil: ${ }^{1} \mathrm{H}$ NMR $\left(\mathrm{CDCl}_{3}\right) \delta 2.25$ (s, 3H), 3.46 (s, 3H), 5.34 (s, 1H), 7.26-7.47 (m, 8H), 7.94-7.98 (m, 2H); ${ }^{13} \mathrm{C} \mathrm{NMR}\left(\mathrm{CDCl}_{3}\right) \delta 13.1,57.2,67.3,79.6,123.3,126.5,127.0$, 127.7, 128.1, 128.5, 130.6, 140.8, 150.08, 150.13 (one sp $^{2}$ carbon missing due to overlap); IR ( $\left.\mathrm{CH}_{2} \mathrm{Cl}_{2}\right)$ 3060, 3028, 2925, 1602, $1485 \mathrm{~cm}^{-1}$; HRMS m/z 404.0279 (calcd for $\mathrm{C}_{19} \mathrm{H}_{17} \mathrm{IO}_{2}$, 404.0273).

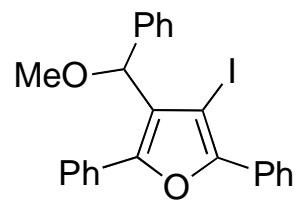

58

\section{3-Iodo-4-[methoxy(phenyl)methyl]-2,5-diphenylfuran (58). Compound 57 (61.6} mg, $0.2 \mathrm{mmol}$ ) was allowed to react with $\mathrm{MeOH}$ (51.2 mg, 8.0 equiv) under our standard reaction conditions for $50 \mathrm{~h}$. The reaction mixture was chromatographed (silica gel, 12:1 hexane/EtOAc) to afford $66.1 \mathrm{mg}$ (71\%) of the indicated compound 58 as a white solid: mp 94-95 ${ }^{\circ} \mathrm{C} ;{ }^{1} \mathrm{H}$ NMR $\left(\mathrm{CDCl}_{3}\right) \delta 3.43$ (s, 3H), 5.75 (s, 1H), 7.25-7.51 (m, 11H), 7.697.72 (m, 2H), 8.11-8.15 (m, 2H); ${ }^{13} \mathrm{C}$ NMR $\left(\mathrm{CDCl}_{3}\right) \delta$ 57.1, 68.2, 123.3, 127.0, 127.1, 127.6, 128.4, 128.59, 128.63, 128.65, 128.67, 130.1, 130.4, 140.2, 151.5, 152.1 (one sp ${ }^{2}$ 
carbon missing due to overlap); IR $\left(\mathrm{CH}_{2} \mathrm{Cl}_{2}\right)$ 3059, 3029, 2927, 1602, $1481 \mathrm{~cm}^{-1}$; HRMS $\mathrm{m} / \mathrm{z} 466.0437$ (calcd for $\mathrm{C}_{24} \mathrm{H}_{19} \mathrm{IO}_{2}, 466.0430$ ).

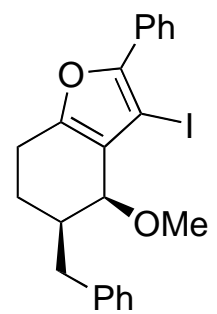

67

cis-5-Benzyl-3-iodo-4-methoxy-2-phenyl-4,5,6,7-tetrahydrobenzofuran (67). Compound 66 (57.2 mg, $0.2 \mathrm{mmol}$ ) was allowed to react with $\mathrm{MeOH}$ (51.2 mg, 8.0 equiv) under our standard reaction conditions for $1 \mathrm{~h}$. The reaction mixture was chromatographed (silica gel, 12:1 hexane/EtOAc) to afford $19.8 \mathrm{mg}$ (22\%) of the indicated compound 67 as a light yellow oil: ${ }^{1} \mathrm{H}$ NMR $\left(\mathrm{CDCl}_{3}\right) \delta 1.76-1.80(\mathrm{~m}, 1 \mathrm{H})$, 1.97-2.04 (m, 2H), 2.51-2.63 (m, 1H), 2.69-2.81 (m, 2H), 2.96-3.03 (m, 1H), 3.63 (s, 3H), 3.98 (s, 1H), 7.21-7.28 (m, 3H), 7.29-7.36 (m, 3H), 7.38-7.43 (m, 2H), 7.91-7.95 (m, 2H); ${ }^{13} \mathrm{C} \mathrm{NMR}\left(\mathrm{CDCl}_{3}\right) \delta$ 23.2, 23.6, 38.6, 43.1, 59.4, 66.1, 73.8, 124.9, 126.2, 126.6, 128.1, 128.5, 128.6, 129.5, 130.8, 140.9, 150.6, 154.3; IR $\left(\mathrm{CH}_{2} \mathrm{Cl}_{2}\right)$ 3025, 2930, 2818, 1629, 1602, 1484, $1459 \mathrm{~cm}^{-1}$; HRMS m/z 444.0593 (calcd for $\mathrm{C}_{22} \mathrm{H}_{21} \mathrm{IO}_{2}$, 444.0586).<smiles>COC1c2c(oc(-c3ccccc3)c2I)CCC1Cc1ccccc1</smiles>

68

trans-5-Benzyl-3-iodo-4-methoxy-2-phenyl-4,5,6,7-tetrahydrobenzofuran (68). The reaction mixture was chromatographed (silica gel, 12:1 hexane/EtOAc) to afford, 
together with 67, $42.1 \mathrm{mg}$ (47\%) of the indicated compound 68 as a light yellow oil: ${ }^{1} \mathrm{H}$ NMR $\left(\mathrm{CDCl}_{3}\right) \delta 1.71-1.78(\mathrm{~m}, 1 \mathrm{H}), 2.13-2.21(\mathrm{~m}, 1 \mathrm{H}), 2.44-2.59(\mathrm{~m}, 3 \mathrm{H})$, 2.67-2.72 (m, 2H), 3.43 (s, 3H), 3.90 (d, $J=0.9 \mathrm{~Hz}, 1 \mathrm{H}), 7.18-7.22$ (m, 2H), 7.24-7.28 (m, 1H), 7.307.37 (m, 3H), 7.39-7.47 (m, 2H), 7.96-8.01 (m, 2H); ${ }^{13} \mathrm{C} \mathrm{NMR}\left(\mathrm{CDCl}_{3}\right) \delta 19.9,22.2$, 36.2, 38.0, 57.2, 65.9, 76.1, 122.3, 126.4, 128.1, 128.6, 128.7, 129.1, 130.8, 140.5, 150.7, 153.2; IR $\left(\mathrm{CH}_{2} \mathrm{Cl}_{2}\right)$ 3025, 2930, 2821, 1615, 1603, 1484, $1453 \mathrm{~cm}^{-1}$; HRMS m/z 444.0592 (calcd for $\mathrm{C}_{22} \mathrm{H}_{21} \mathrm{IO}_{2}, 444.0586$ ).

Representative procedure for the PhSeCl-induced cyclizations. To the mixture of appropriate 2-(1-alkynyl)-2-alken-1-one (0.2 mmol), $\mathrm{PhSeCl}$ (3.0 equiv) and $\mathrm{NaHCO}_{3}$ (3.0 equiv), was added a solution of nucleophile (10 equiv) in $\mathrm{CH}_{3} \mathrm{CN}$ (2.0 mL). The resulting mixture was stirred at room temperature for $1 \mathrm{~h}$ unless otherwise specified. The mixture was diluted with ether $(25 \mathrm{~mL})$, washed with brine $(15 \mathrm{~mL})$ and dried $\left(\mathrm{MgSO}_{4}\right)$. The solvent was removed under reduced pressure and the residue was purified by flash chromatography on silica gel.

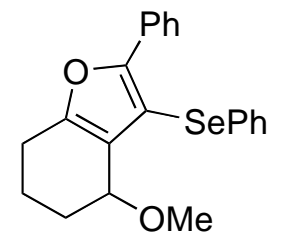

5

\section{4-Methoxy-2-phenyl-3-phenylseleno-4,5,6,7-tetrahydrobenzofuran (5).}

Compound 1 (39.2 mg, $0.2 \mathrm{mmol}$ ) was allowed to react with MeOH (64 mg, 10 equiv) under our standard reaction conditions for $1 \mathrm{~h}$. The reaction mixture was chromatographed (silica gel, 9:1 hexane/EtOAc) to afford $34.2 \mathrm{mg}$ (45\%) of the indicated compound 5 as a light yellow oil: ${ }^{1} \mathrm{H}$ NMR $\left(\mathrm{CDCl}_{3}\right) \delta 1.44-1.57(\mathrm{~m}, 1 \mathrm{H}), 1.82-1.91(\mathrm{~m}$, 1H), 2.00-2.14 (m, 2H), 2.55-2.68 (m, 1H), 2.72-2.82 (m, 1H), $3.31(\mathrm{~s}, 3 \mathrm{H}), 4.13$ (t, $J=$ 
$2.7 \mathrm{~Hz}, 1 \mathrm{H})$, 7.11-7.24 (m, 3H), 7.26-7.38 (m, 5H), 7.93-7.98 (m, 2H); ${ }^{13} \mathrm{C} \mathrm{NMR}\left(\mathrm{CDCl}_{3}\right)$

$\delta$ 18.2, 23.4, 27.3, 57.1, 71.3, 103.6, 124.1, 126.1, 126.6, 128.1, 128.5, 129.2, 129.4, 130.9, 132.8, 153.6, 154.4; IR $\left(\mathrm{CH}_{2} \mathrm{Cl}_{2}\right)$ 3056, 2938, 1603, 1577, $1478 \mathrm{~cm}^{-1}$; HRMS m/z 384.0636 (calcd for $\mathrm{C}_{21} \mathrm{H}_{20} \mathrm{O}_{2} \mathrm{Se}, 384.0629$ ).

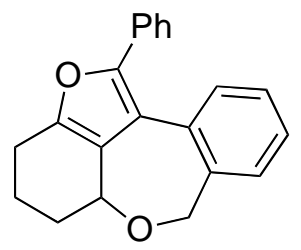

79

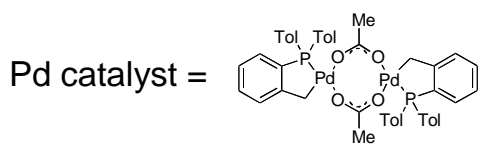

This compound was prepared by following a procedure from the literaure. ${ }^{8}$ The Pd catalyst (11.8 mg, $5 \mathrm{~mol} \%), \mathrm{Cs}_{2} \mathrm{CO}_{3}(195 \mathrm{mg}, 0.6 \mathrm{mmol})$, furan 27 (87.1 mg, $0.20 \mathrm{mmol}$ ) and DMA (2.5 mL), were placed in a vial. The resulting mixture was heated under a $\mathrm{N}_{2}$ atmosphere at $100{ }^{\circ} \mathrm{C}$ for $17 \mathrm{~h}$. The mixture was allowed to cool to room temperature, diluted with diethyl ether (30 mL), washed with satd aq $\mathrm{NH}_{4} \mathrm{Cl}(15 \mathrm{~mL})$, dried $\left(\mathrm{MgSO}_{4}\right)$, and filtered. The solvent was removed under reduced pressure and the residue was purified by column chromatography (9:1 hexane/EtOAc) on silica gel to afford $46.6 \mathrm{mg}$ (76\%) of the indicated compound 79 as a white solid: mp $156-157{ }^{\circ} \mathrm{C} ;{ }^{1} \mathrm{H}$ NMR $\left(\mathrm{CDCl}_{3}\right)$ $\delta$ 1.81-2.05 (m, 4H), 2.55-2.64 (m, 1H), 2.73-2.80 (m, 1H), 4.49-4.56 (m, 2H), 4.76 (d, $J$ = 9.0 Hz, 1H), 7.24-7.35 (m, 5H), 7.42-7.45 (m, 1H), 7.54-7.56 (m, 1H), 7.69-7.71 (m, $2 \mathrm{H}) ;{ }^{13} \mathrm{C} \mathrm{NMR}\left(\mathrm{CDCl}_{3}\right) \delta 20.7,23.4,30.8,68.2,68.8,120.5,121.0,125.8,127.6,127.70$, 127.73, 128.7, 128.8, 131.1, 131.5, 134.7, 137.5, 146.0, 152.1; IR $\left(\mathrm{CH}_{2} \mathrm{Cl}_{2}\right)$ 3057, 2945, 2856, 1603, $1497 \mathrm{~cm}^{-1}$; HRMS m/z 302.1312 (calcd for $\mathrm{C}_{21} \mathrm{H}_{18} \mathrm{O}_{2}, 302.1307$ ). 


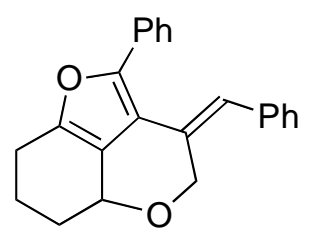

80

This compound was prepared by following a procedure from the literaure. ${ }^{9} \mathrm{Pd}(\mathrm{OAc})_{2}$ (4.5 mg, $10 \mathrm{~mol} \%$ ), $\mathrm{PPh}_{3}$ (10.5 mg, $20 \mathrm{~mol} \%$ ), n-Bu ${ }_{4} \mathrm{NCl}$ (55.3 mg, $0.2 \mathrm{mmol}$ ), 88\% $\mathrm{HCO}_{2} \mathrm{H}$ (31.4 mg, $0.6 \mathrm{mmol}$ ), piperidine (68.2 mg, $0.8 \mathrm{mmol}$ ), furan 28 (90.7 mg, 0.20 mmol) and $\mathrm{CH}_{3} \mathrm{CN}(5 \mathrm{~mL})$, were placed in a vial. The resulting mixture was heated under a $\mathrm{N}_{2}$ atmosphere at $60{ }^{\circ} \mathrm{C}$ for $14 \mathrm{~h}$. The mixture was allowed to cool to room temperature, diluted with diethyl ether (30 mL), washed with satd aq $\mathrm{NH}_{4} \mathrm{Cl}(15 \mathrm{~mL})$, dried $\left(\mathrm{MgSO}_{4}\right)$, and filtered. The solvent was removed under reduced pressure and the residue was purified by column chromatography (9:1 hexane/EtOAc) on silica gel to afford $49.2 \mathrm{mg}(75 \%)$ of the indicated compound $\mathbf{8 0}$ as a yellow oil: ${ }^{1} \mathrm{H}$ NMR $\left(\mathrm{CDCl}_{3}\right) \delta$ 1.35-1.48 (m, 1H), 1.78-1.92 (m, 1H), 2.13-2.29 (m, 2H), 2.58-2.77 (m, 2H), 4.48 (dd, $J$ $=2.1,14.4 \mathrm{~Hz}, 1 \mathrm{H}), 4.52-4.59(\mathrm{~m}, 1 \mathrm{H}), 5.03(\mathrm{dd}, J=1.2,14.1 \mathrm{~Hz}, 1 \mathrm{H}), 7.11-7.15(\mathrm{~m}$, 2H), 7.19-7.26 (m, 1H), 7.29-7.36 (m, 4H), 7.39-7.55 (m, 2H), 7.76-7.80 (m, $2 \mathrm{H}) ;{ }^{13} \mathrm{C}$ $\operatorname{NMR}\left(\mathrm{CDCl}_{3}\right) \delta 20.7,22.9,29.4,67.5,71.9,114.8,121.9,123.4,127.1,127.7,128.2$, 128.5, 128.8, 129.4, 132.6, 136.8, 147.9, 148.4; IR $\left(\mathrm{CH}_{2} \mathrm{Cl}_{2}\right)$ 3056, 3021, 2946, 2846, 1673, 1599, $1486 \mathrm{~cm}^{-1}$; HRMS m/z 328.1471 (calcd for $\mathrm{C}_{23} \mathrm{H}_{20} \mathrm{O}_{2}$, 328.1463).

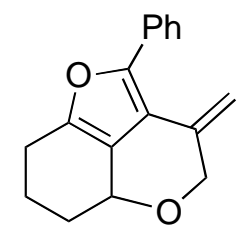

81 
This compound was prepared by following a procedure from the literaure. ${ }^{10} \mathrm{Pd}(\mathrm{OAc})_{2}$ (4.5 mg, $10 \mathrm{~mol} \%), \mathrm{PPh}_{3}(10.5 \mathrm{mg}, 20 \mathrm{~mol} \%), \mathrm{Et}_{3} \mathrm{~N}$ (81 mg, $\left.0.8 \mathrm{mmol}\right)$ and furan 24 (76.9 mg, $0.20 \mathrm{mmol})$ in $\mathrm{CH}_{3} \mathrm{CN}(4.5 \mathrm{~mL}$ ) were refluxed for $3 \mathrm{~h}$. The mixture was allowed to cool to room temperature, diluted with diethyl ether (30 mL), washed with satd aq $\mathrm{NH}_{4} \mathrm{Cl}(15 \mathrm{~mL})$, dried $\left(\mathrm{MgSO}_{4}\right)$, and filtered. The solvent was removed under reduced pressure and the residue was purified by column chromatography (12:1 hexane/EtOAc) on silica gel to afford $23.3 \mathrm{mg}$ (46\%) of the indicated compound $\mathbf{8 1}$ as a light yellow solid: mp 68-70 ${ }^{\circ} \mathrm{C} ;{ }^{1} \mathrm{H}$ NMR $\left(\mathrm{CDCl}_{3}\right) \delta$ 1.34-1.44 (m, 1H), 1.76-1.91 (m, 1H), 2.09-2.25 (m, 2H), 2.56-2.72 (m, 2H), 4.31-4.43 (m, 2H), 4.48-4.53 (m, 1H), 4.89 (s, 1H), 5.66 (s, 1H), 7.26-7.31 (m, 1H), 7.39 (t, $J=5.9 \mathrm{~Hz}, 2 \mathrm{H}), 7.68-7.71(\mathrm{~m}, 2 \mathrm{H}) ;{ }^{13} \mathrm{C}$ $\operatorname{NMR}\left(\mathrm{CDCl}_{3}\right) \delta 20.6,22.7,29.2,72.0,72.2,106.8,113.6,121.5,127.3,128.0,128.5$, 132.0, 135.2, 147.7, 149.0; IR $\left(\mathrm{CH}_{2} \mathrm{Cl}_{2}\right)$ 3007, 2946, 2848, 1669, 1602, $1443 \mathrm{~cm}^{-1}$; HRMS m/z 252.1154 (calcd for $\mathrm{C}_{17} \mathrm{H}_{16} \mathrm{O}_{2}, 252.1150$ ).

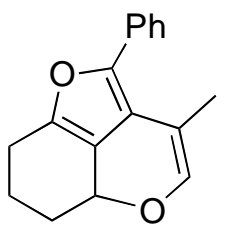

82

The reaction mixture was chromatographed (silica gel, 12:1 hexane/EtOAc) to afford, together with $\mathbf{8 1}$, $7.6 \mathrm{mg}(15 \%)$ of the indicated compound $\mathbf{8 2}$ as a colorless oil: ${ }^{1} \mathrm{H}$ NMR $\left(\mathrm{CDCl}_{3}\right) \delta$ 1.60-1.69 (m, 1H), 1.79-1.88 (m, 1H), $1.92(\mathrm{~d}, J=1.2 \mathrm{~Hz}, 3 \mathrm{H}), 2.14-2.21(\mathrm{~m}$, 1H), 2.29-2.35 (m, 1H), 2.63-2.68 (m, 2H), 4.87-4.92 (m, 1H), 6.29 (d, $J=1.2 \mathrm{~Hz}, 1 \mathrm{H})$, 7.27-7.31 (m, 1H), 7.35-7.41 (m, 2H), 7.56-7.60 (m, 2H); IR $\left(\mathrm{CH}_{2} \mathrm{Cl}_{2}\right)$ 3013, 2941, 2851, 1669, 1600, $1445 \mathrm{~cm}^{-1}$; HRMS m/z 252.1154 (calcd for $\mathrm{C}_{17} \mathrm{H}_{16} \mathrm{O}_{2}, 252.1150$ ). 


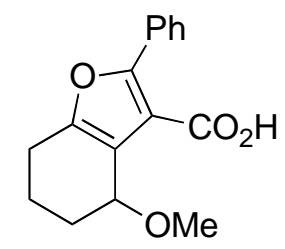

83

$\mathrm{Pd}\left(\mathrm{PCy}_{3}\right)_{2}$ (8.4 mg, $\left.10 \mathrm{~mol} \%\right), \mathrm{CsO}_{2} \mathrm{CCMe}_{3}$ (117 mg, $20 \mathrm{~mol} \%$ ), furan 3 (88.5 mg, $0.25 \mathrm{mmol}), \mathrm{H}_{2} \mathrm{O}$ (9 mg, $\left.0.5 \mathrm{mmol}\right)$ and DMF (6 mL) were placed in a vial. The resulting mixture was heated under a $\mathrm{CO}$ atmosphere $(1 \mathrm{~atm})$ at $110{ }^{\circ} \mathrm{C}$ for $7 \mathrm{~h}$. The mixture was allowed to cool to room temperature, diluted with diethyl ether (30 mL), washed with satd aq $\mathrm{NH}_{4} \mathrm{Cl}(15 \mathrm{~mL})$, dried $\left(\mathrm{MgSO}_{4}\right)$, and filtered. The solvent was removed under reduced pressure and the residue was purified by column chromatography (2:1 hexane/EtOAc) on silica gel to afford $36.2 \mathrm{mg}$ (53\%) of the indicated compound 83 as a white solid: mp 159-160 ${ }^{\circ} \mathrm{C} ;{ }^{1} \mathrm{H}$ NMR $\left(\mathrm{CDCl}_{3}\right) \delta 1.78-1.90$ (m, 2H), 1.97-2.10 (m, 2H), 2.51-2.66 (m, 1H), 2.69-2.80 (m, 1H), 3.50 (s, 3H), 4.64 (t, $J=3.7$ Hz, 1H), 7.36-7.44 (m, 3H), 7.83-7.89 (m, 2H); ${ }^{13} \mathrm{C} \mathrm{NMR}\left(\mathrm{CDCl}_{3}\right) \delta 18.5,23.2,26.8,56.5,72.2,112.8$, 118.6, 128.3, 128.8, 129.5, 130.0, 153.5, 157.9, 166.8; IR $\left(\mathrm{CH}_{2} \mathrm{Cl}_{2}\right)$ 3059, 2930, 1711, 1681, $1491 \mathrm{~cm}^{-1}$; HRMS m/z 272.1052 (calcd for $\mathrm{C}_{16} \mathrm{H}_{16} \mathrm{O}_{4}, 272.1049$ ).

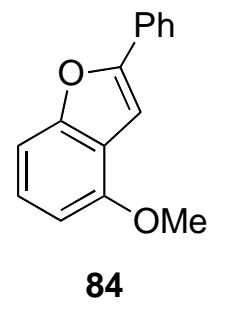

This compound was prepared by following a procedure from the literaure. ${ }^{11}$ Furan 2 (45.6 mg, $0.20 \mathrm{mmol})$ and DDQ (85 mg, $0.34 \mathrm{mmol})$ in benzene $(6.5 \mathrm{~mL})$ were refluxed for $6 \mathrm{~h}$. The mixture was allowed to cool to room temperature, filtrated, washed with 10 
$\% \mathrm{Na}_{2} \mathrm{CO}_{3}(6 \mathrm{~mL})$, dried $\left(\mathrm{MgSO}_{4}\right)$, and filtered. The solvent was removed under reduced pressure and the residue was purified by column chromatography (12:1 hexane/EtOAc) on silica gel to afford $17.7 \mathrm{mg}$ (40\%) of the indicated compound 84 as a colorless oil: ${ }^{1} \mathrm{H}$ NMR $\left(\mathrm{CDCl}_{3}\right) \delta 3.97$ (s, 3H), 6.67 (dd, $\left.J=0.9,7.5 \mathrm{~Hz}, 1 \mathrm{H}\right), 7.13-7.26$ (m, 3H), 7.317.36 (m, 1H), 7.41-7.48 (m, 2H), 7.83-7.87 (m, 2H); ${ }^{13} \mathrm{C} \mathrm{NMR}\left(\mathrm{CDCl}_{3}\right) \delta$ 55.8, 99.0, 103.5, 104.7, 119.8, 125.0, 125.2, 128.5, 129.0, 130.8, 153.7, 154.9, 156.3; IR $\left(\mathrm{CH}_{2} \mathrm{Cl}_{2}\right)$ 2961, 1607, 1486, $1252 \mathrm{~cm}^{-1}$; HRMS m/z 224.0841 (calcd for $\mathrm{C}_{15} \mathrm{H}_{12} \mathrm{O}_{2}, 224.0837$ ).

\section{References}

1. Yao, T.; Zhang, X.; Larock, R. C. J. Am. Chem. Soc. 2004, 126, 2677.

2. Johnson, C. R.; Adams, J. P.; Braun, M. P.; Senanayake, C. B. W.; Wovkulich, P. M.; Uskokovic, M. R. Tetrahedron Lett. 1992, 33, 917.

3. Miller, M. W.; Johnson, C. R. J. Org. Chem. 1997, 62, 1582.

4. Mayasundari, A.; Young, D. G. J. Tetrahedron Lett. 2001, 42, 203.

5. Zhang, F.; Li, Y. Synthesis, 1993, 565.

6. Lautens, M.; Maddess, M. L.; Sauer, E. L. O.; Quellet, S. G. Org. Lett. 2002, 4, 83.

7. Dai, W.-M.; Wu, J.; Fong, K. C.; Lee, M. Y. H.; Lau, C. W. J. Org. Chem. 1999, 64, 5062.

8. Hennings, D. D.; Iwasa, S.; Rawal, V. H. J. Org. Chem. 1997, 62, 2.

9. Grigg, R.; Loganathan, V.; Sridharan, V.; Stevenson, P.; Sukirthalingam, S.;

Worakun, T. Tetrahedron 1996, 52, 11479.

10. Knight, S. D.; Overman, L. E. Heterocycles 1994, 39, 497. 
11. Zambias, R. A.; Caldwell, C. G.; Kopka, I. E.; Hammond, M. L. J. Org. Chem. 1988, 53, 4135. 


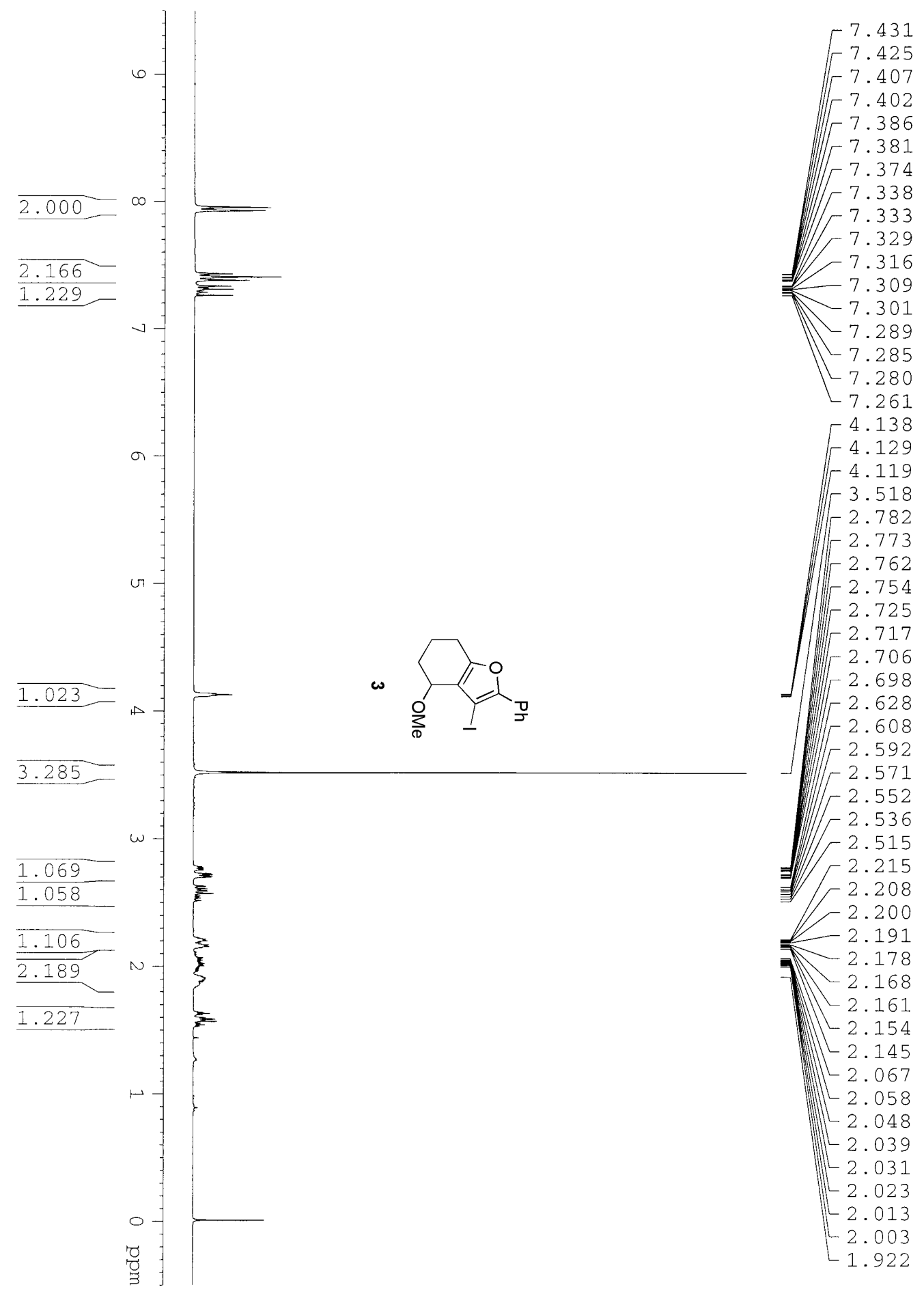




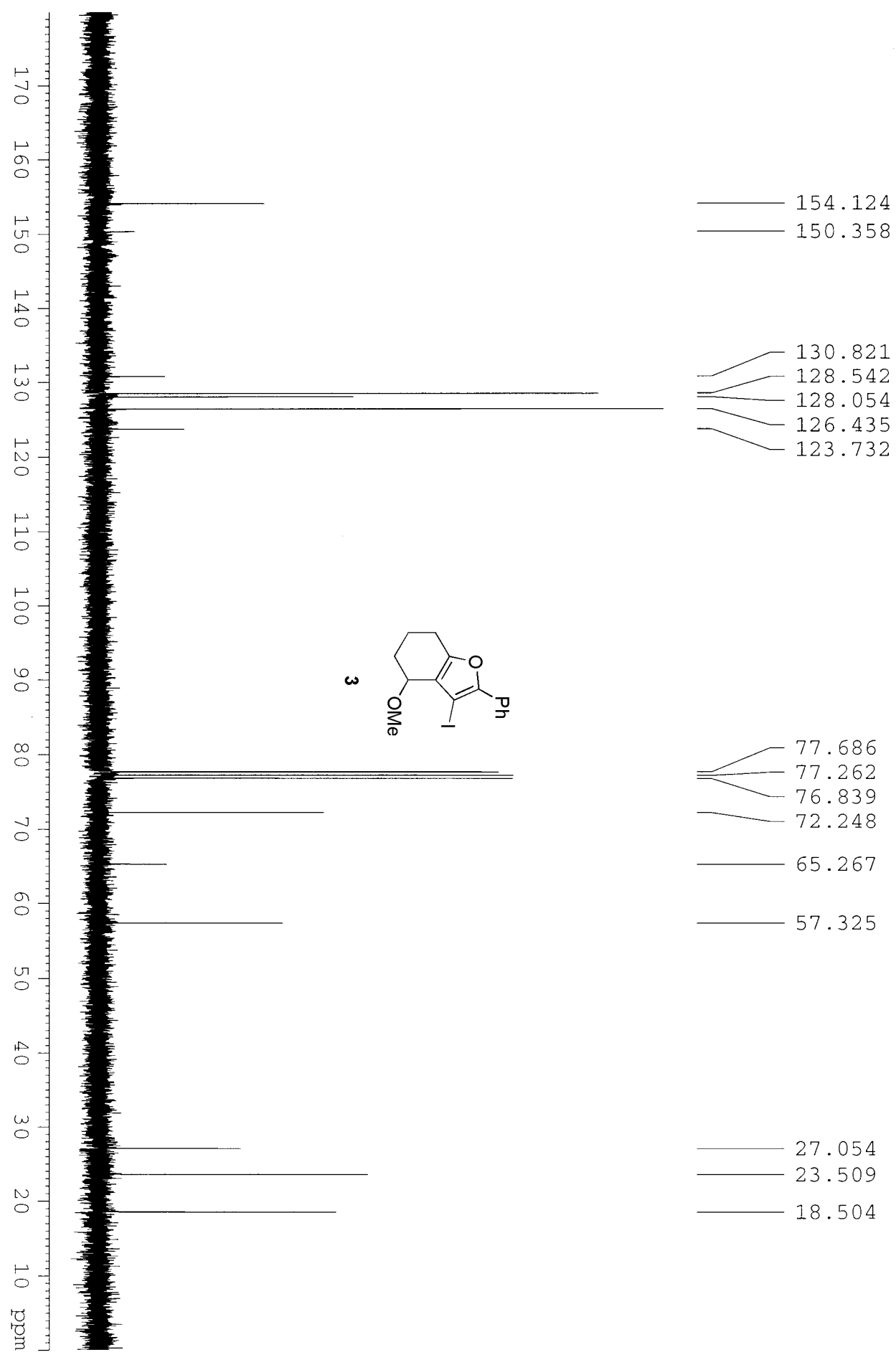



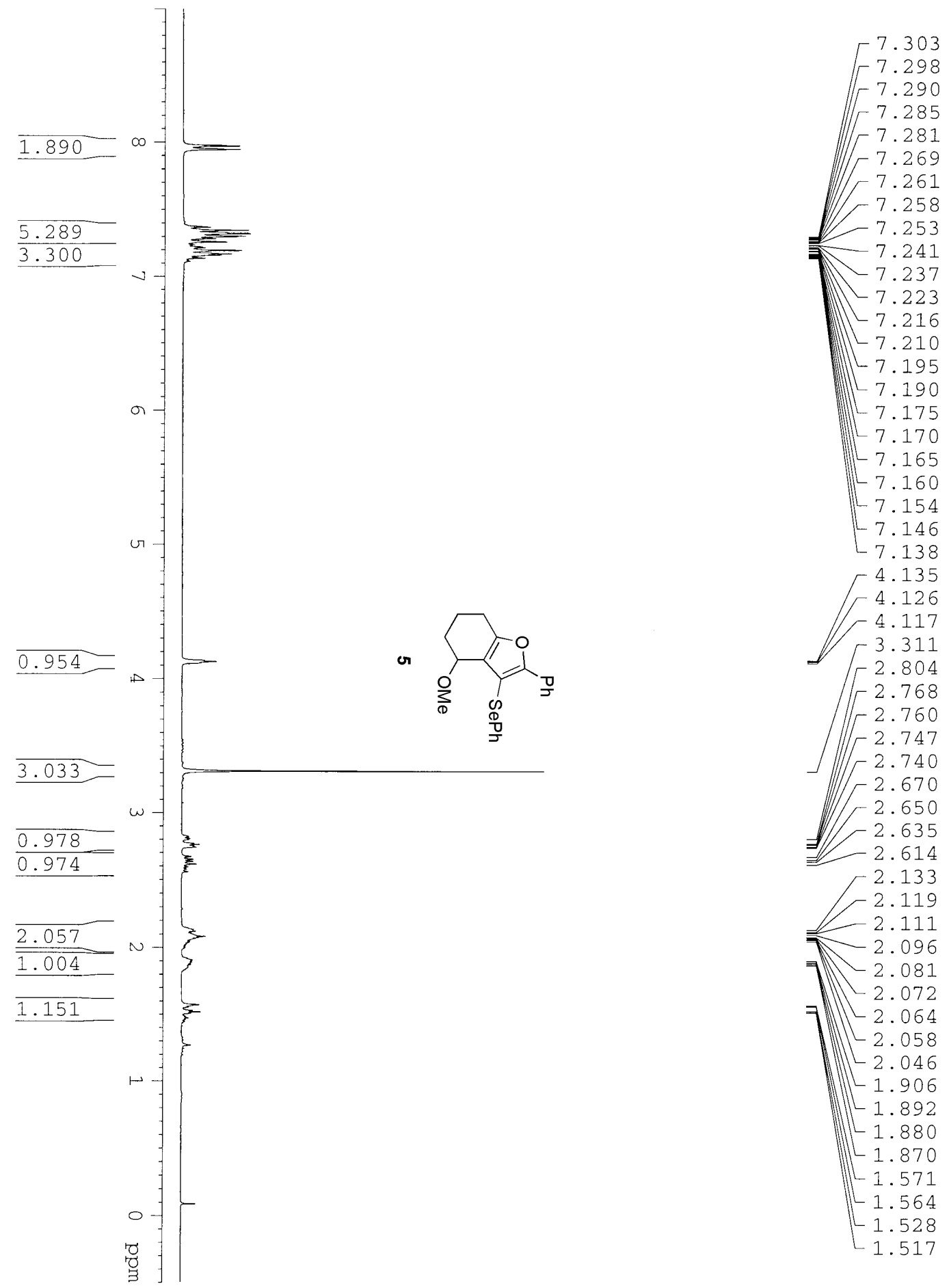

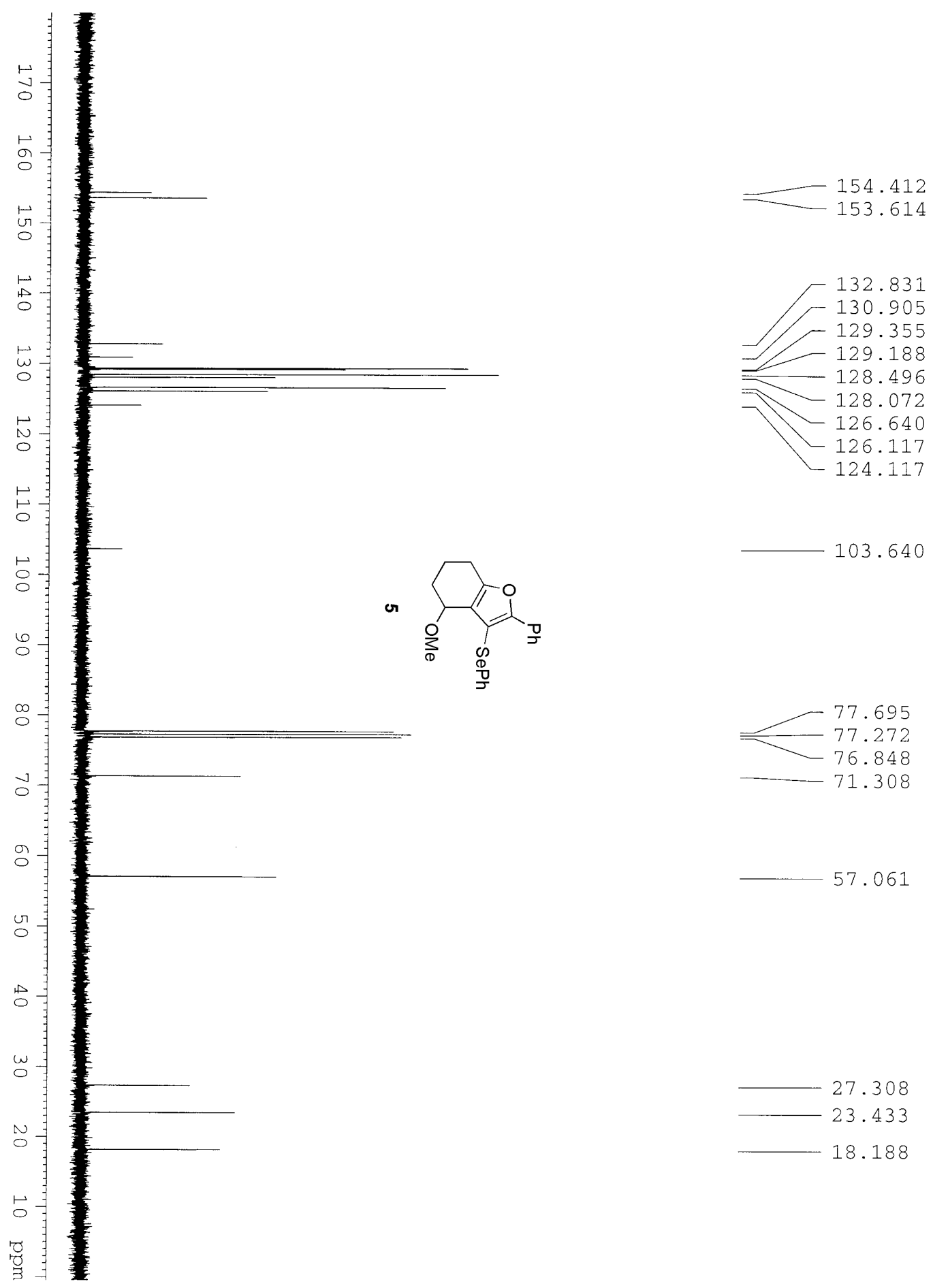

103.640

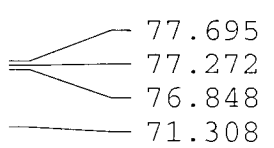

57.061

27.308

23.433

18.188 

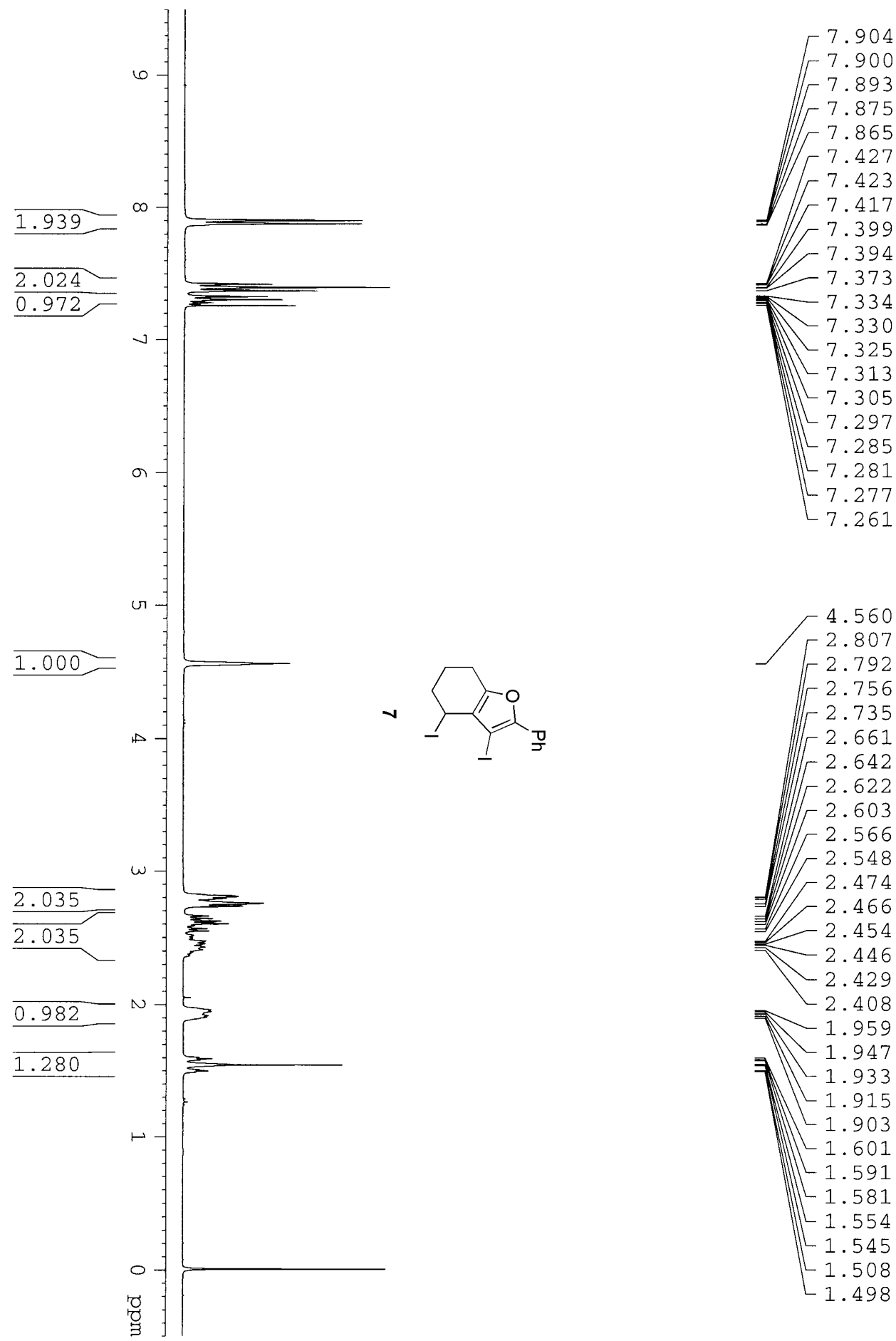

$-2.756$

$-2.735$

$-2.661$

$-2.642$

$-2.622$

$-2.603$

$-2.566$

$-2.548$

-2.474
-2.466

-2.454
-2.446

$-2.429$

$-2.408$

$\$ \begin{array}{r}2.959 \\ 1.954\end{array}$

$-1.947$

$\mathbb{A}-1.933$

$-1.915$

$-1.903$

$-1.601$

$-1.591$

ᄂ 1.581

$-1.554$

1.545

1.508

$-1.498$ 


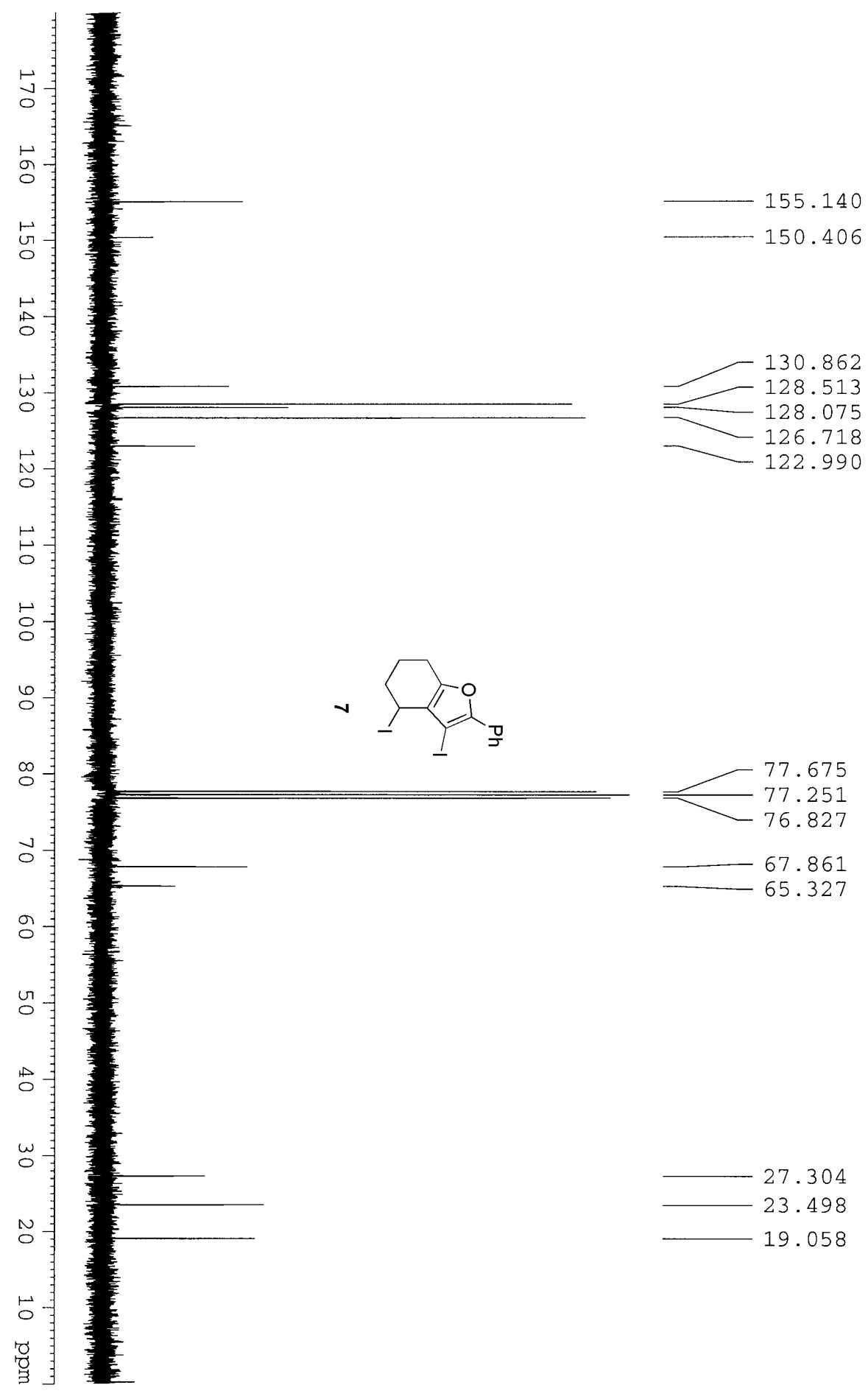



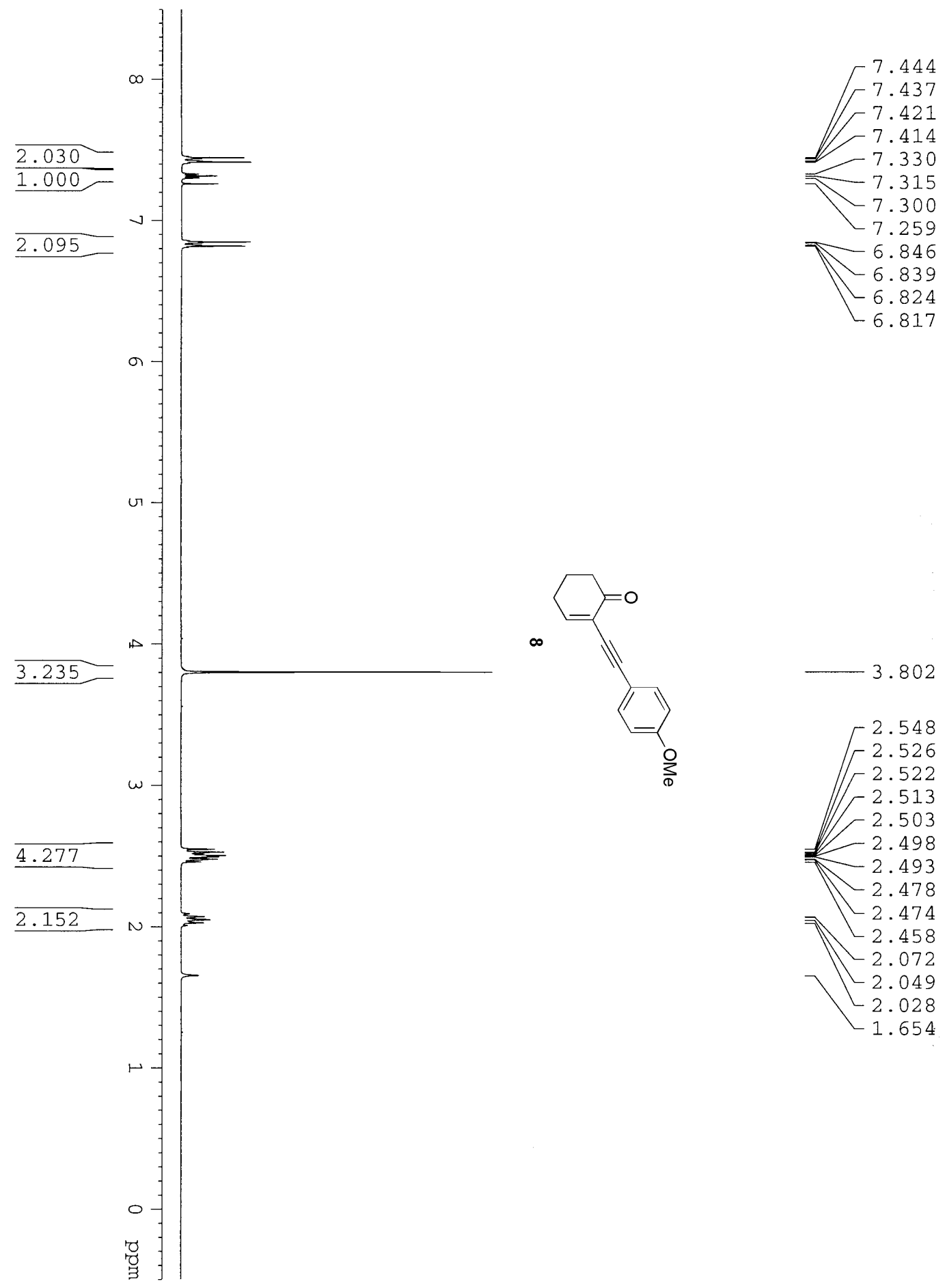

$-3.802$

2.548

2.526

$\begin{array}{r}2.522 \\ \hline 2.513\end{array}$

2.513
-2.503

$\begin{array}{r}2.503 \\ -2.498 \\ \hline\end{array}$

-2.498
-2.493
-2.478

$-2.478$

$\approx-2.474$

$-2.458$

$-2.072$

$-2.049$

$-2.028$

$-1.654$ 

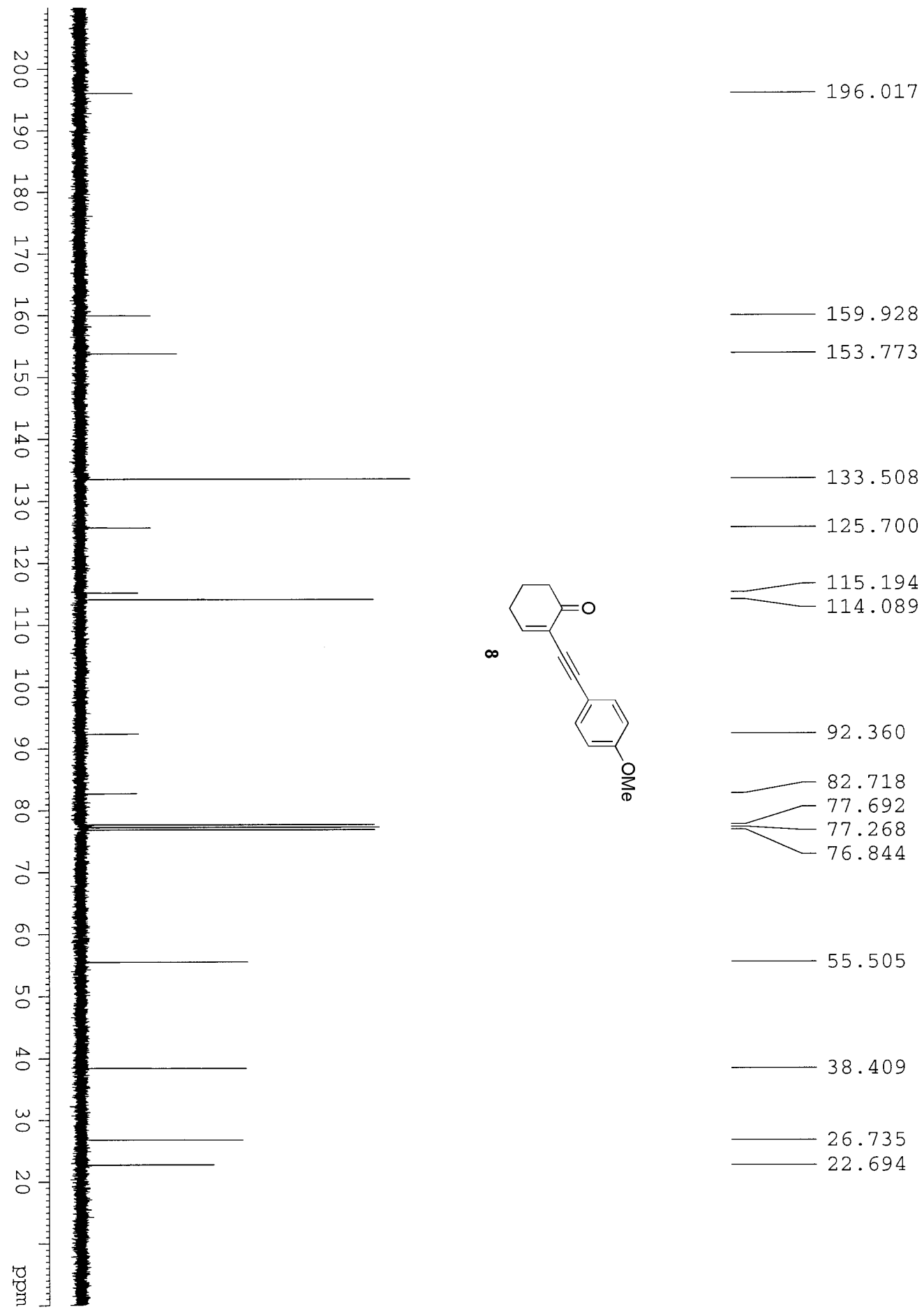

55.505

38.409

26.735 22.694 

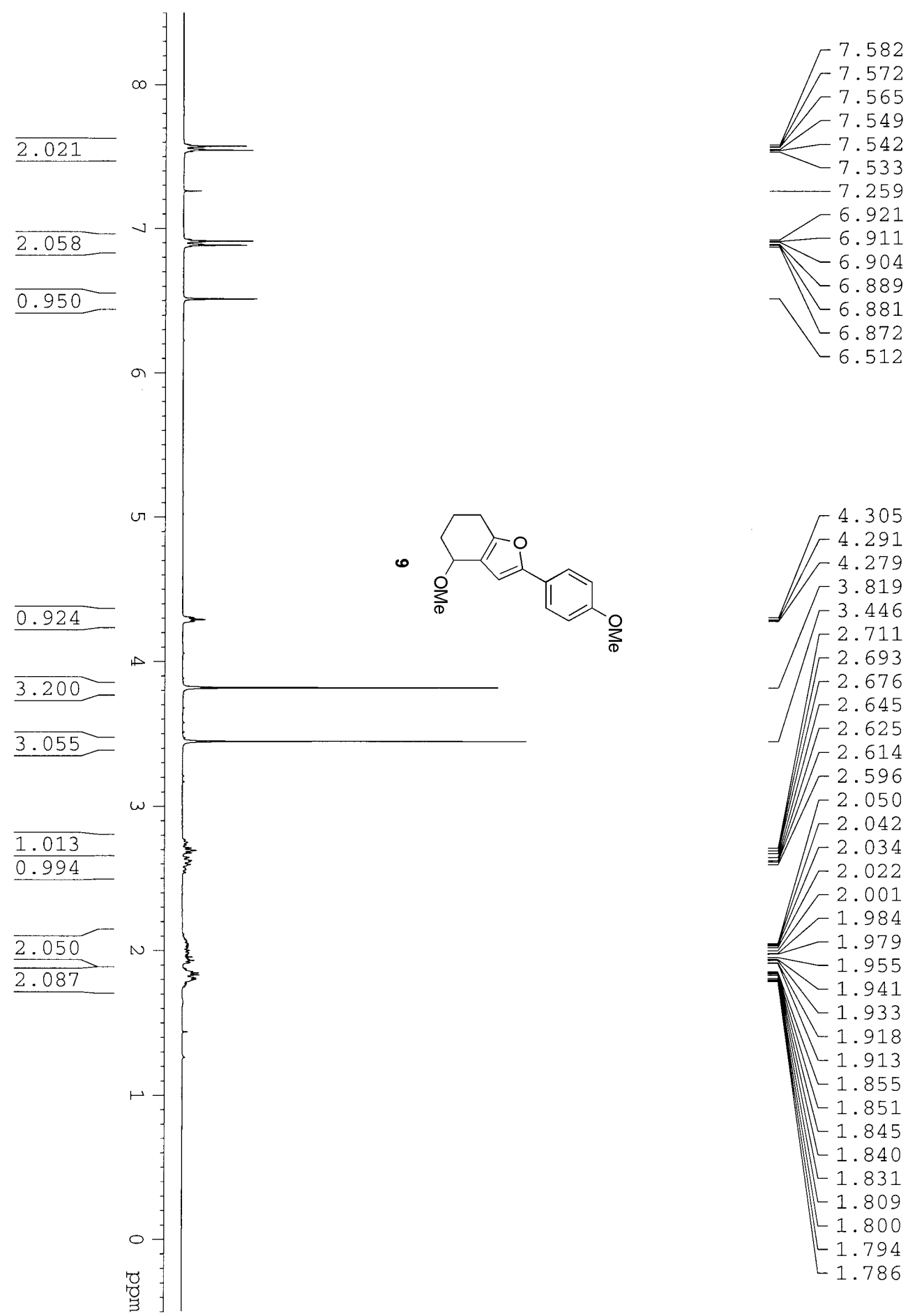

r 4.291

4.279

$\left\lceil=\begin{array}{l}3.819 \\ 3.446\end{array}\right.$

$\lceil 2.711$

$-2.693$

]-2.676

$-2.645$

]-2.625

$-2.614$

$-2.596$

$-2.050$

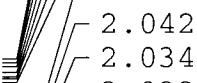

$\digamma^{2} .022$

$-2.001$

1.001
1.984
-1.979

$-1.955$

$7-1.941$

$-1.933$

$-1.918$

$\lfloor 1.913$

$-1.855$

L 1.851

$-1.845$

1.840

- 1.831

$[1.809$

1.800

L 1.794

$L_{1.786}$ 

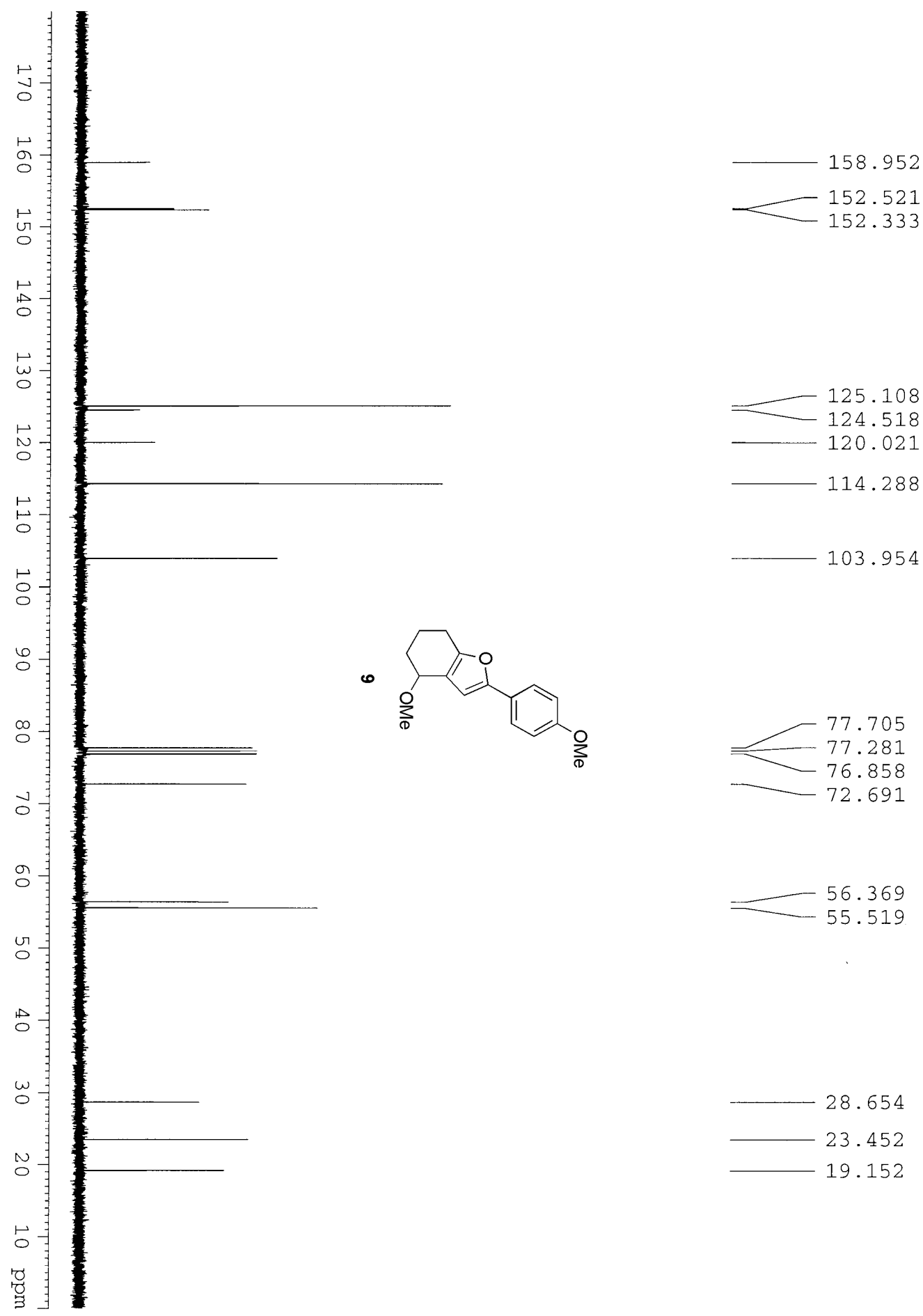

120.021

114.288

103.954

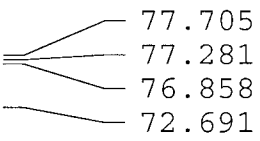

-
- 6.369

55.519

28.654

23.452

19.152 

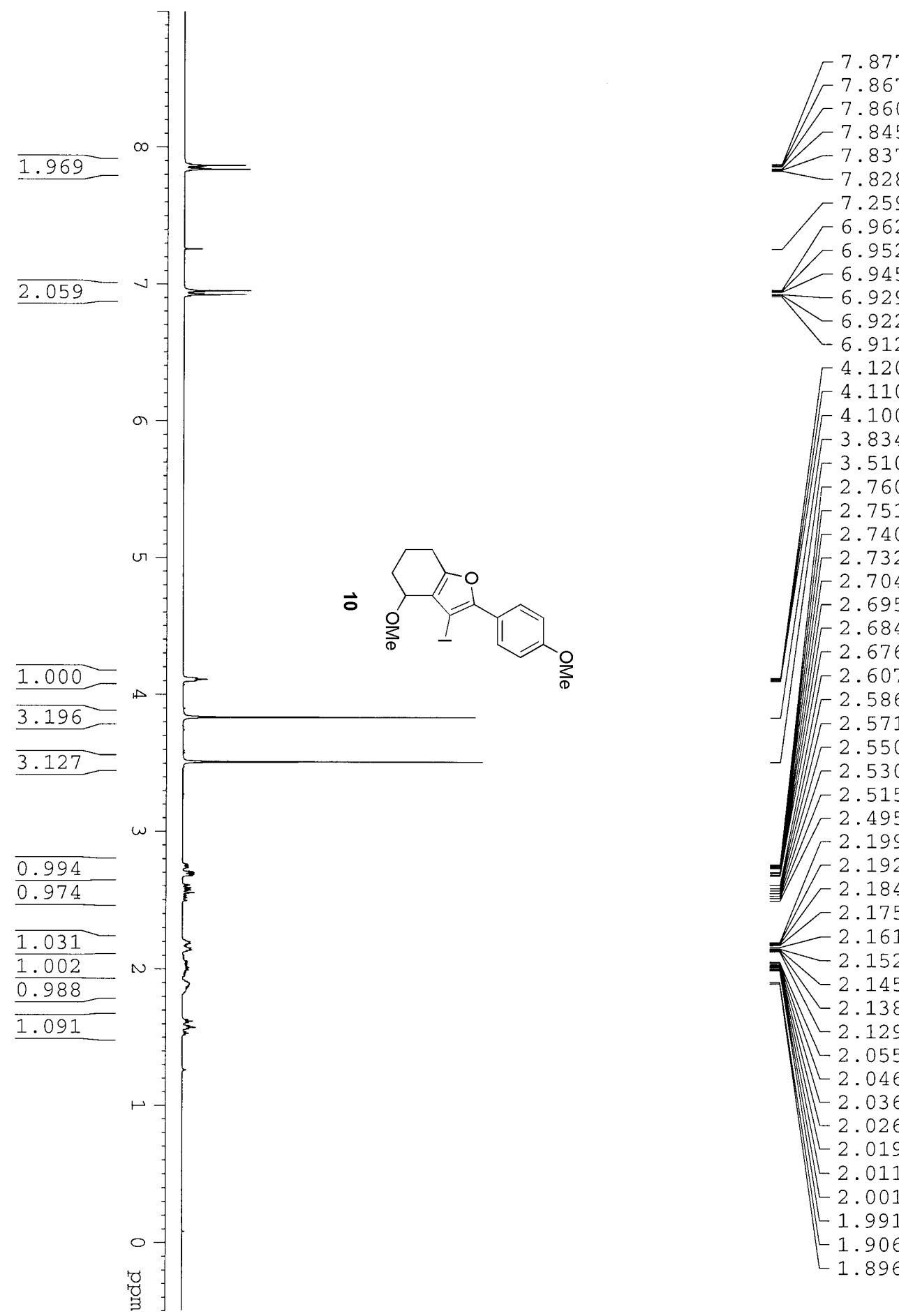

7.259

7.259
6.962
6.952
6.945
6.942
6.929
6.922

$-6.912$

$-4.120$

$-4.110$

$-4.100$

$-3.834$

3.510

$-2.760$

$-2.751$

$-2.740$

$-2.732$

$-2.704$

$-2.695$

$\left[\begin{array}{l}2.684 \\ 2.676 \\ -2.607\end{array}\right.$

- $\left[\begin{array}{l}2.607 \\ 2.586\end{array}\right.$

- $\begin{aligned} & 2.571 \\ & 2.586\end{aligned}$

$-2.550$

$\mp 2.530$

F 2.495

2.199

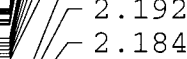

$-2.175$

$-2.161$

$\$-2.152$

$7 \backslash 2.145$

$-2.138$

2.129

$-2.055$

$-2.046$

$-2.036$

L2.026

$-2.019$

L 2.011

2.001

$-1.991$

$-1.906$

1.896 

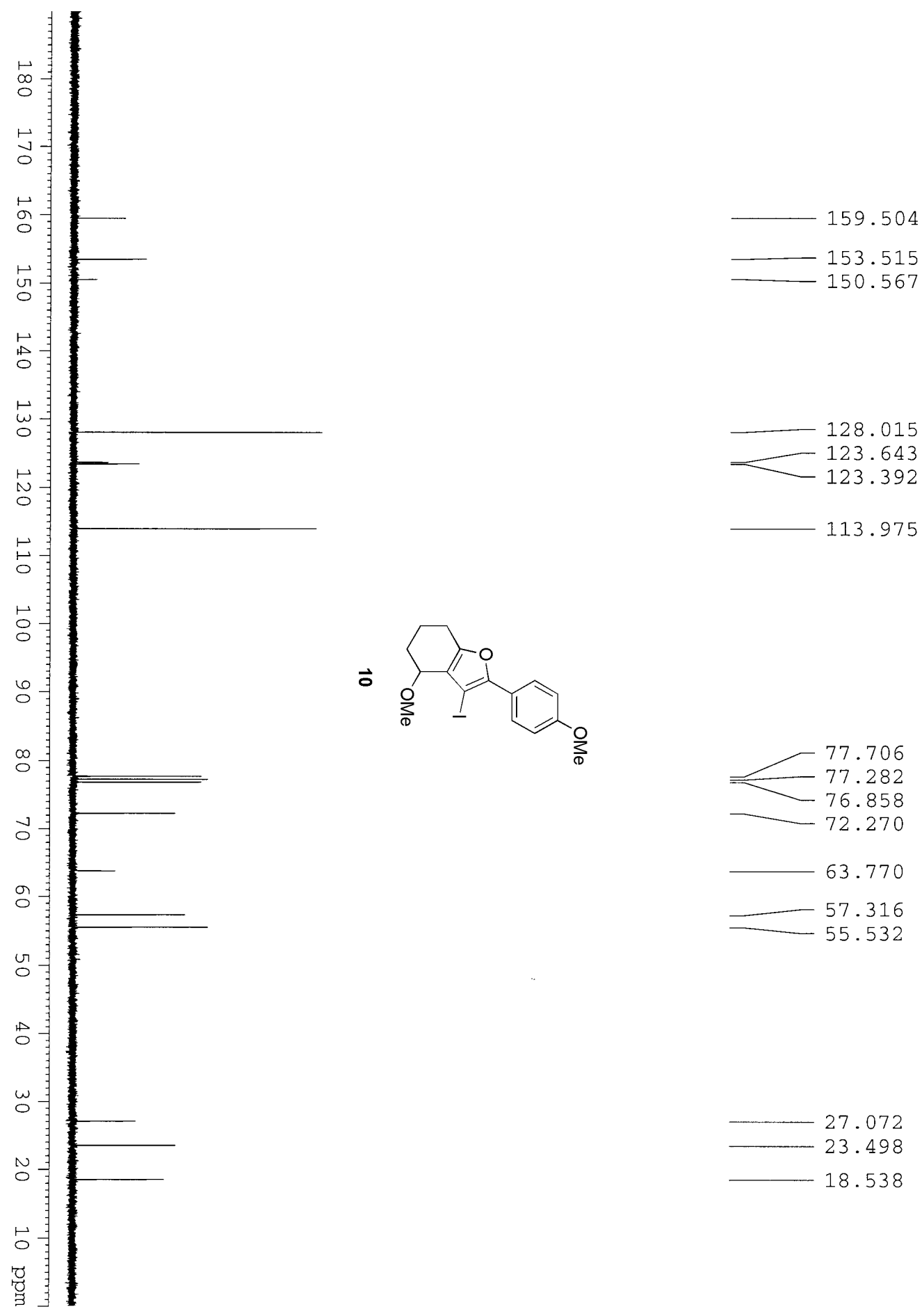

113.975
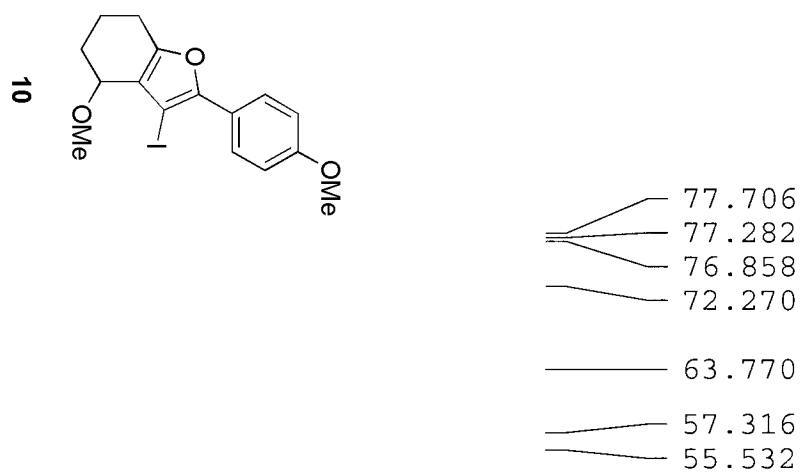

27.072

23.498

18.538 

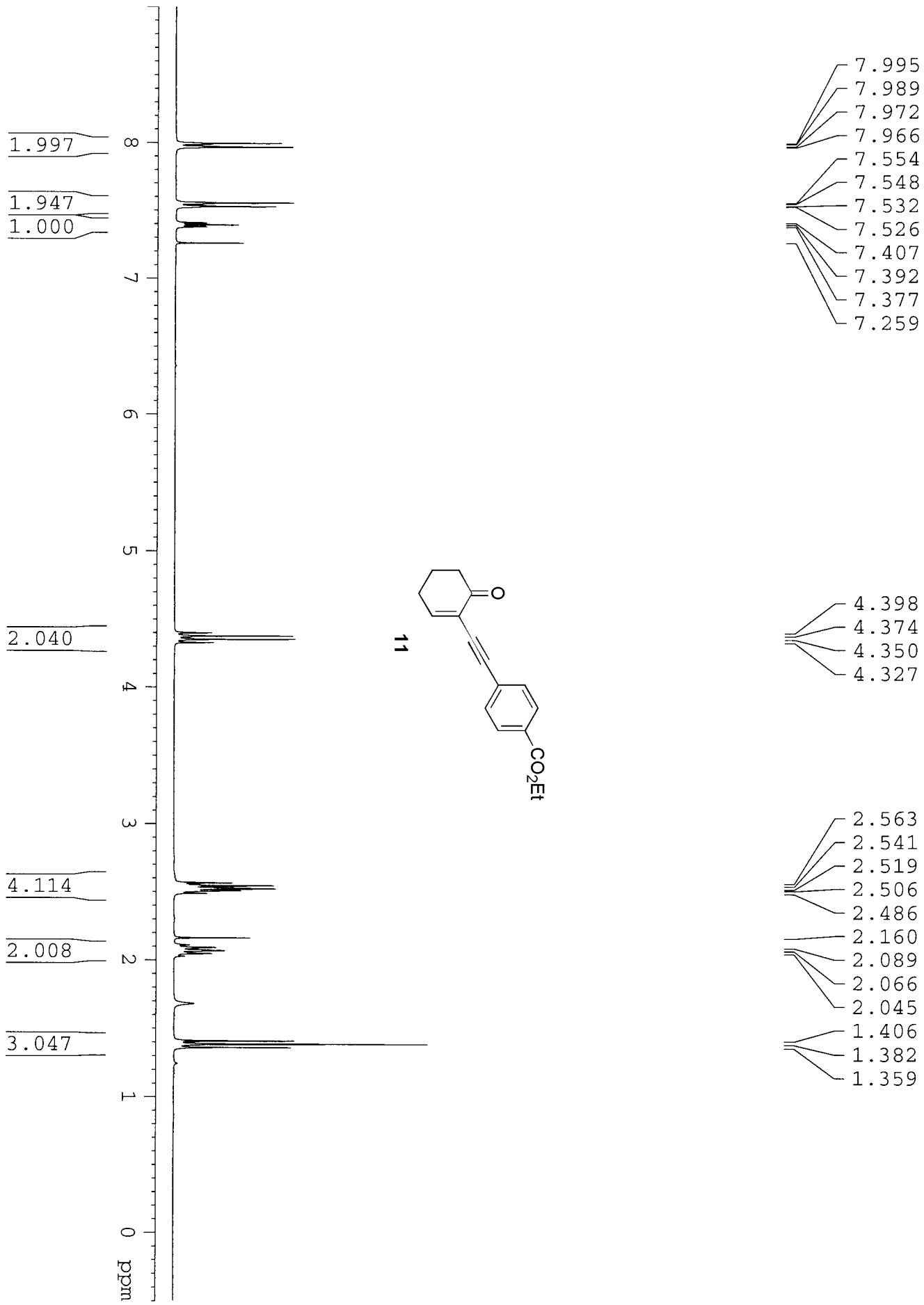

$-2.160$

$-2.089$

$-2.066$

$-2.045$

$-1.406$

$-1.382$

1.359 

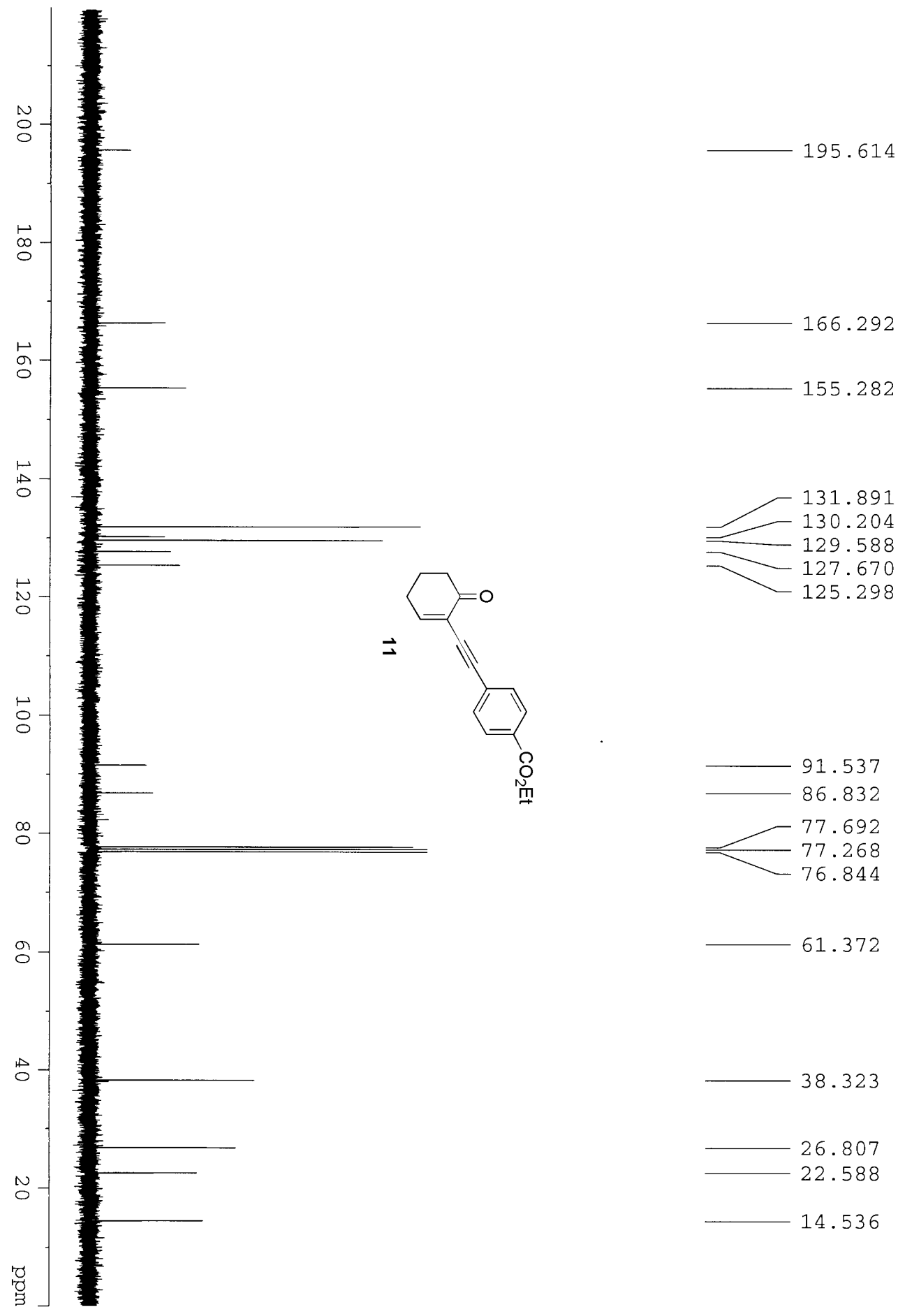

61.372

38.323

26.807

22.588

14.536 

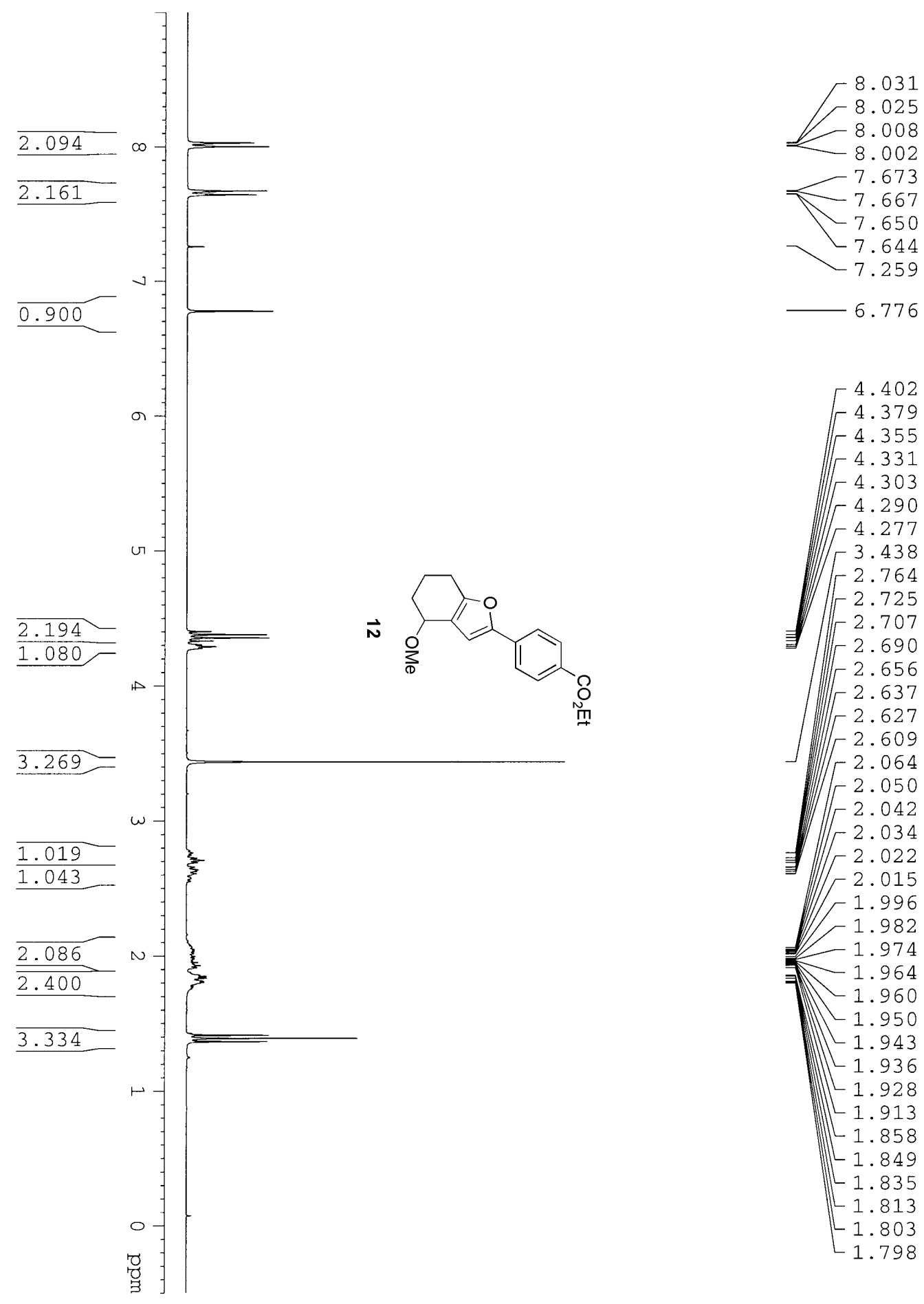

4.355

$-4.303$

$-4.290$

$-4.277$

$-3.438$

$\left[\begin{array}{r}2.764 \\ -2.725\end{array}\right.$

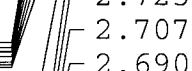

$-2.656$

$-2.637$

$\lceil 2.627$

]$\left[\begin{array}{l}2.609 \\ -2.064\end{array}\right.$

$\| \quad-2.050$

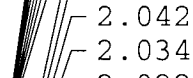

当 2.022

$\digamma^{2.015}$

$-1.996$

$-1.982$

$-1.974$

$-1.964$

$-1.960$

$-1.950$

$-1.943$

$-1.936$

$\llcorner 1.928$

$-1.913$

L 1.858

$-1.849$

$-1.835$

$-1.813$

$-1.803$

1.798 


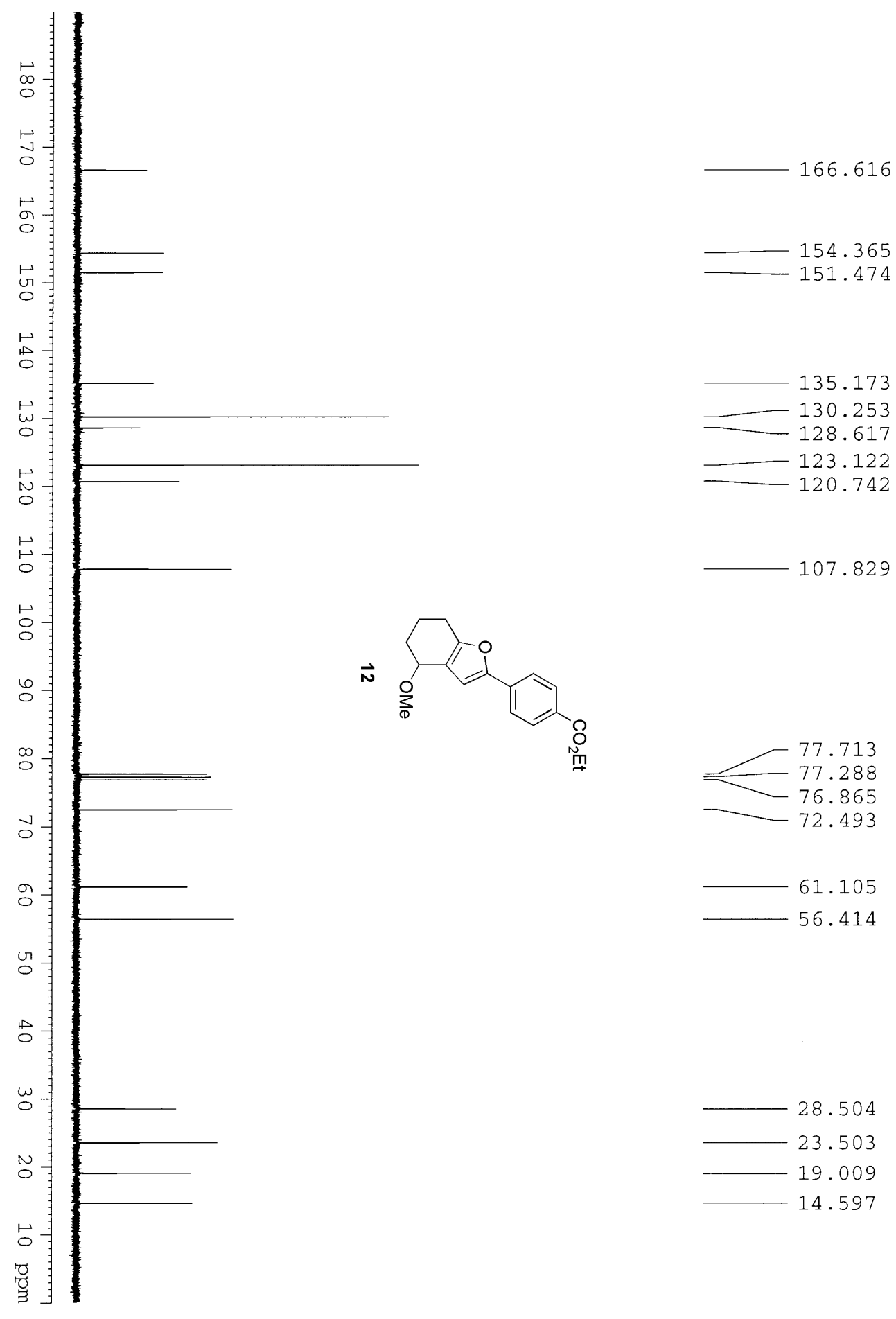



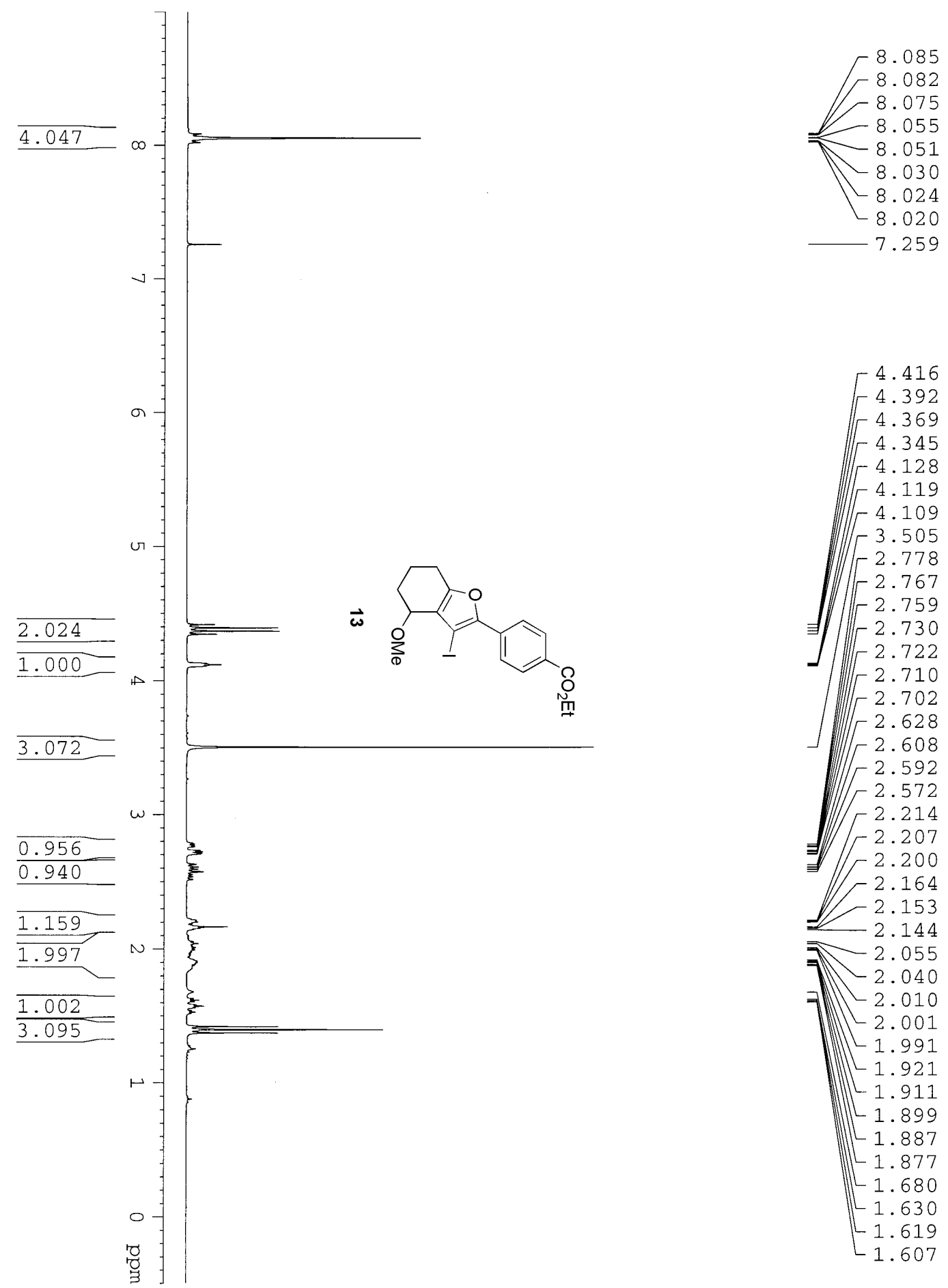

$-4.416$

$-4.392$

4.369

4.345

4.128

4.119

$-4.109$

$-3.505$

$-2.778$

$-2.767$

I -2.759

$\exists-2.730$

] $\left[\begin{array}{l}2.722 \\ 2.710\end{array}\right.$

$\lceil 2.710$

2.702

$\lceil-2.628$

$-2.608$

-2.592

$\begin{array}{r}2.572 \\ \hline 2.214\end{array}$

$\digamma^{2.214}$

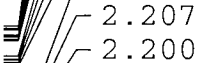

$-2.164$

$-2.153$

2.144

$\$-2.055$

$\longrightarrow-2.040$

]$\quad[-2.010$

- 1.991

$-1.921$

$-1.911$

L 1.899

1.887

$-1.877$

1.680

$-1.630$

1.619

- 1.607 


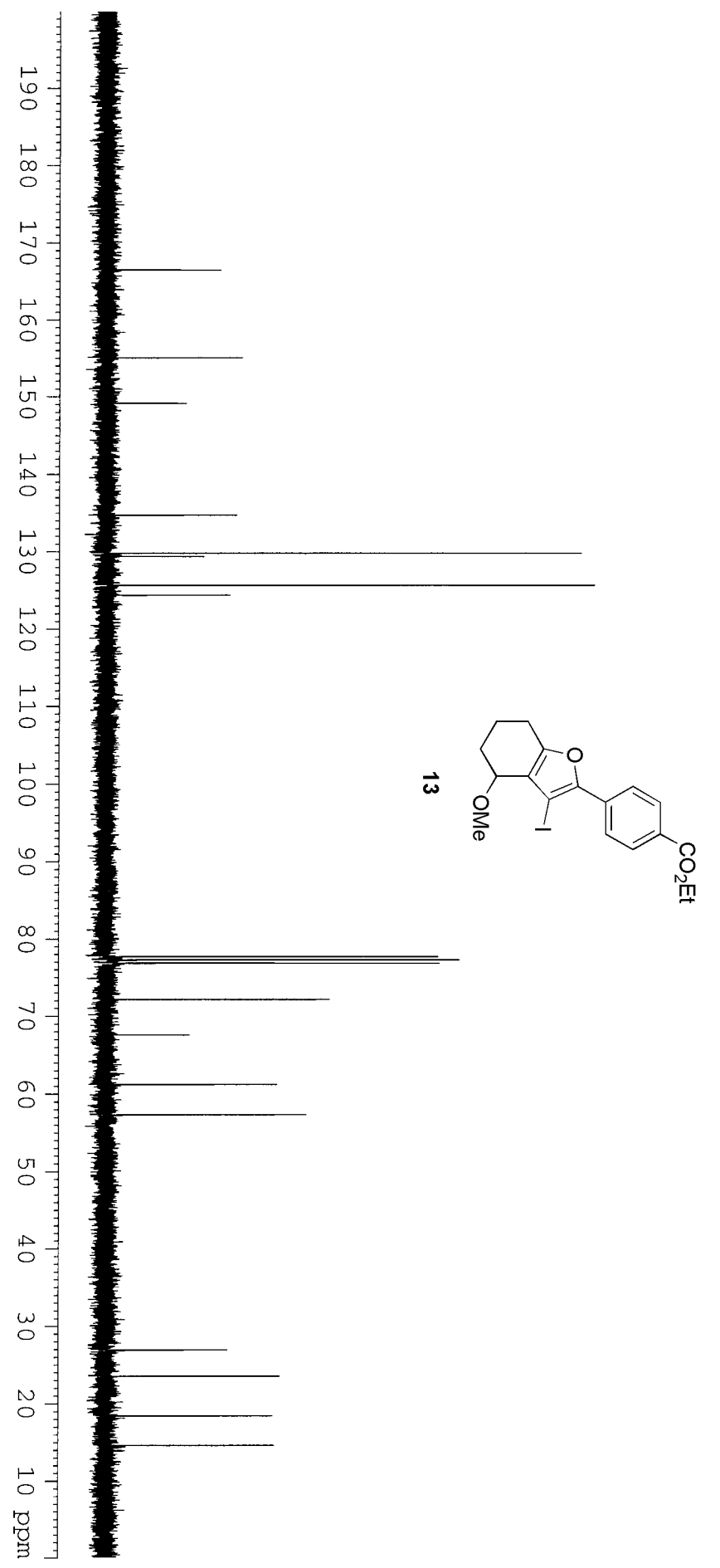

166.516

155.070

149.181

134.744
-129.860

$\simeq \quad 129.411$

$=125.680$

124.418

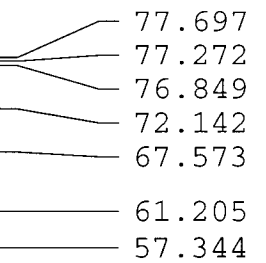

26.922

23.521

18.413

14.591 

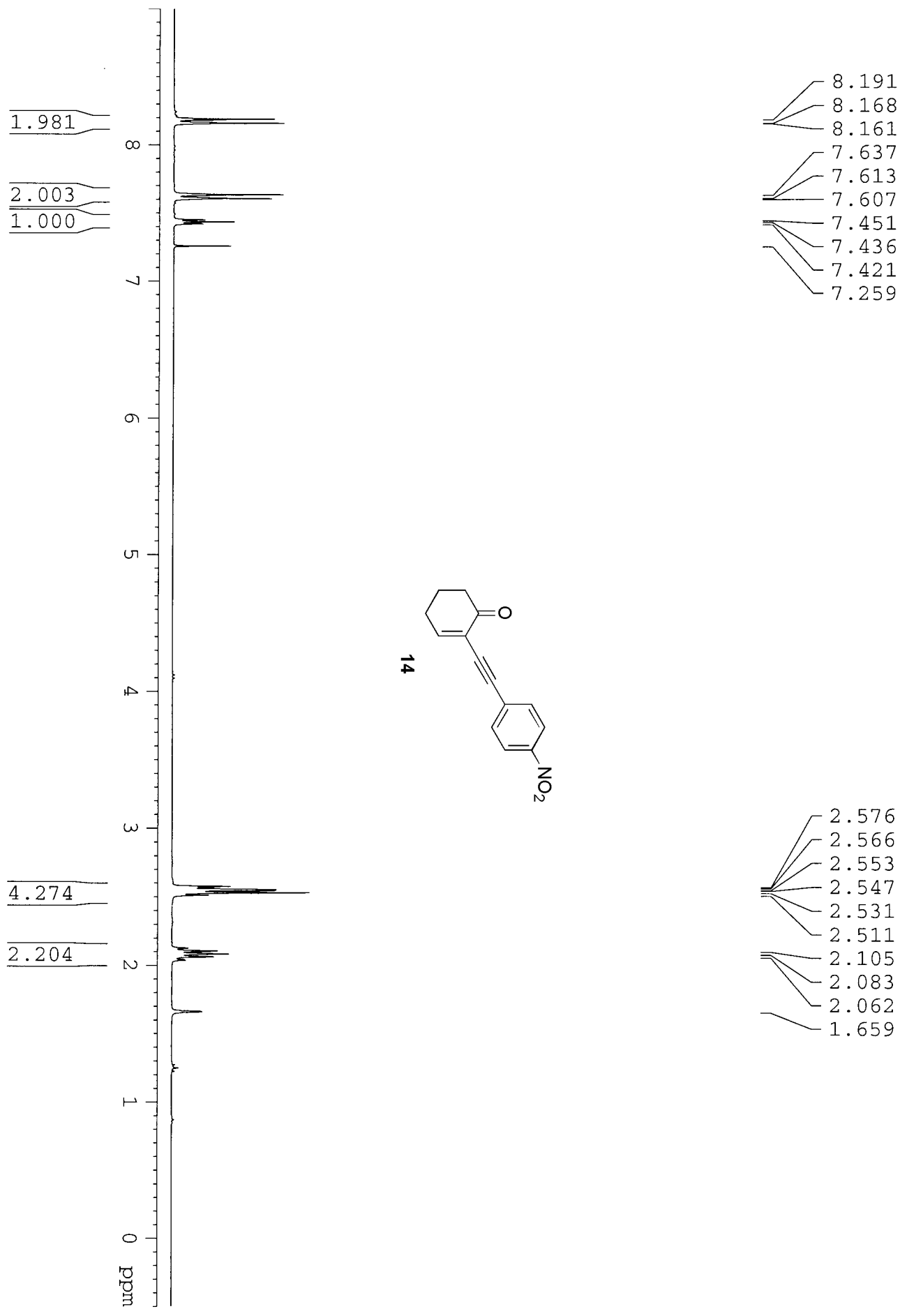

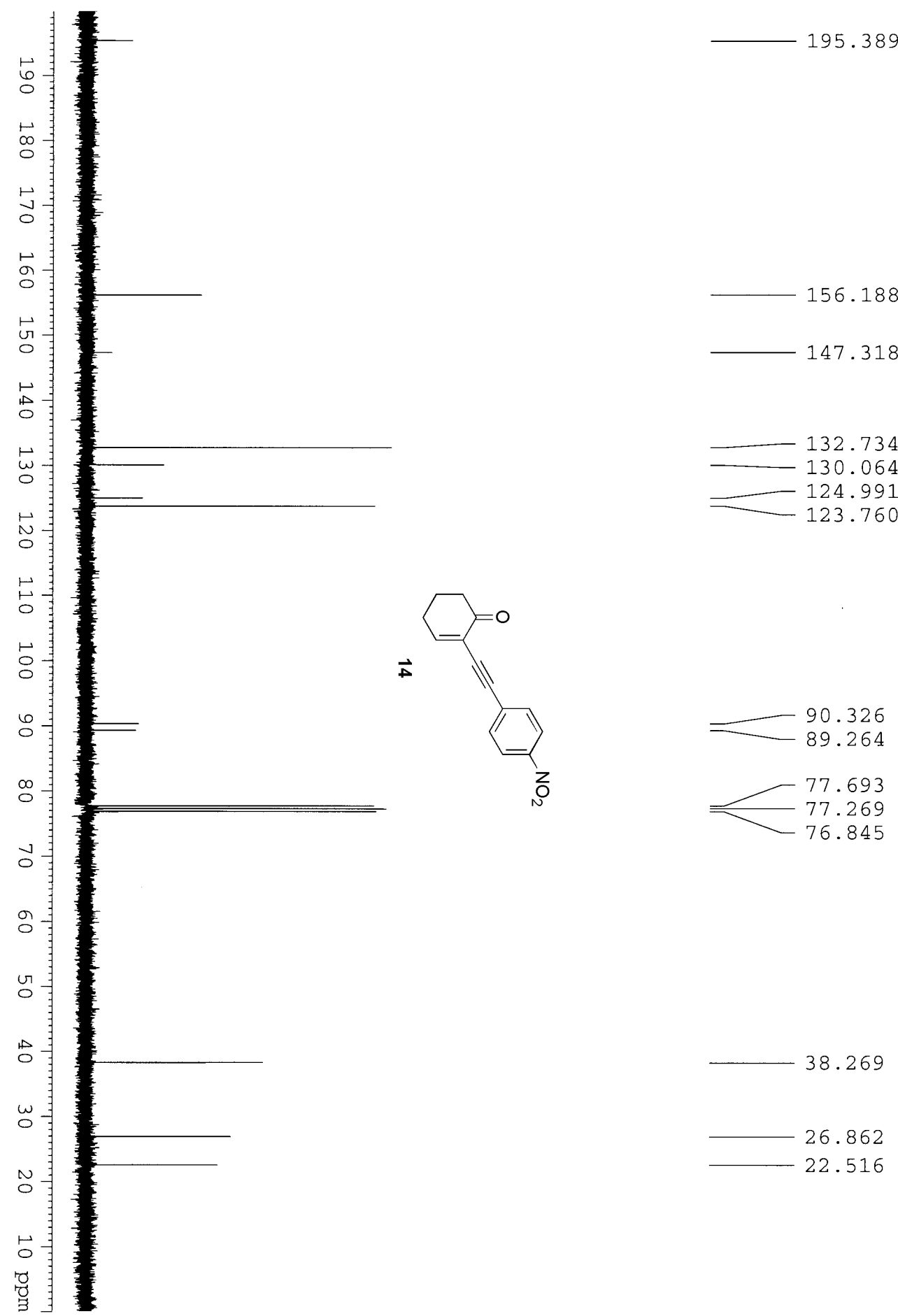

$-90.326$

89.264

77.693

77.269

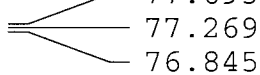

38.269

26.862

22.516 

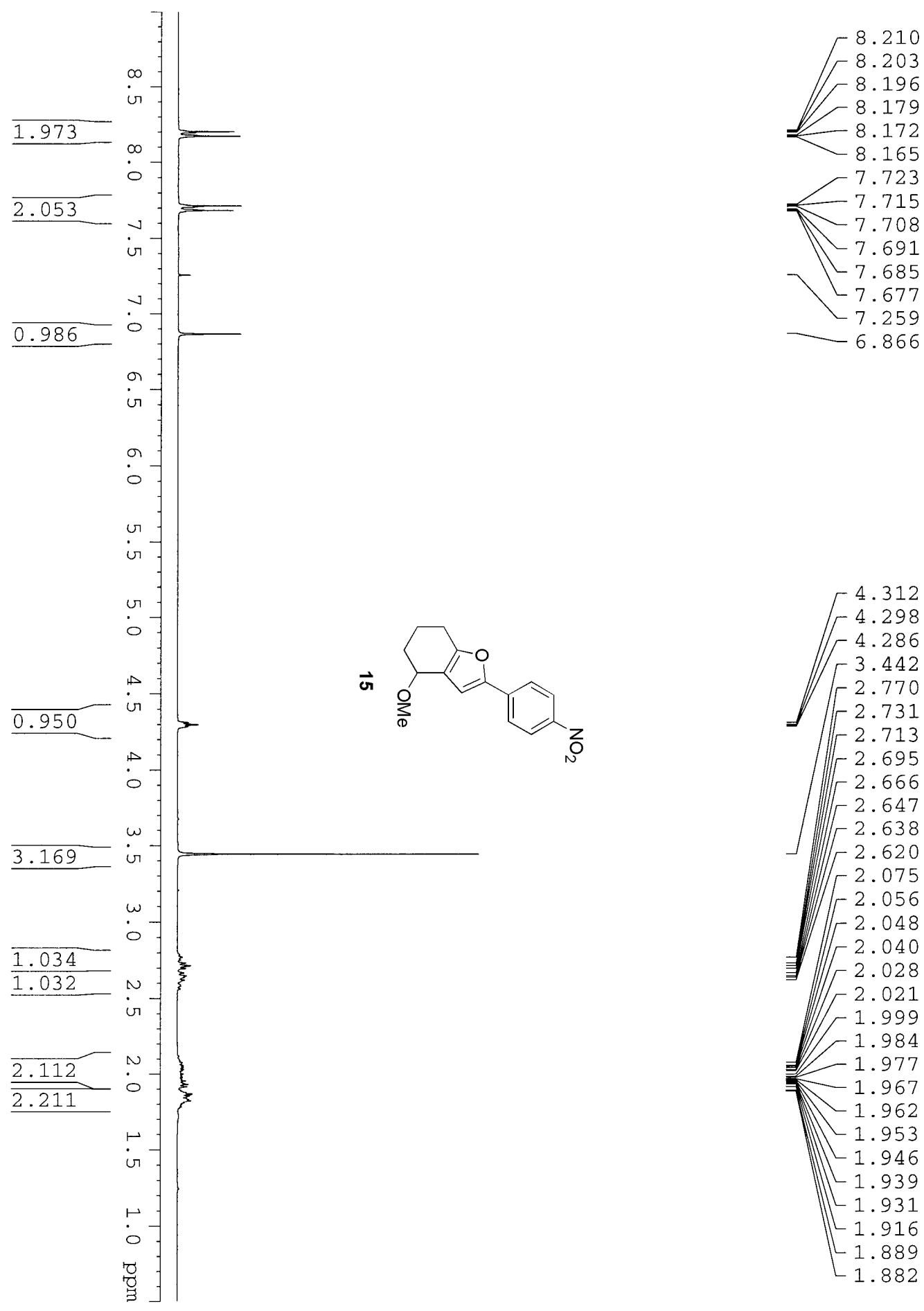


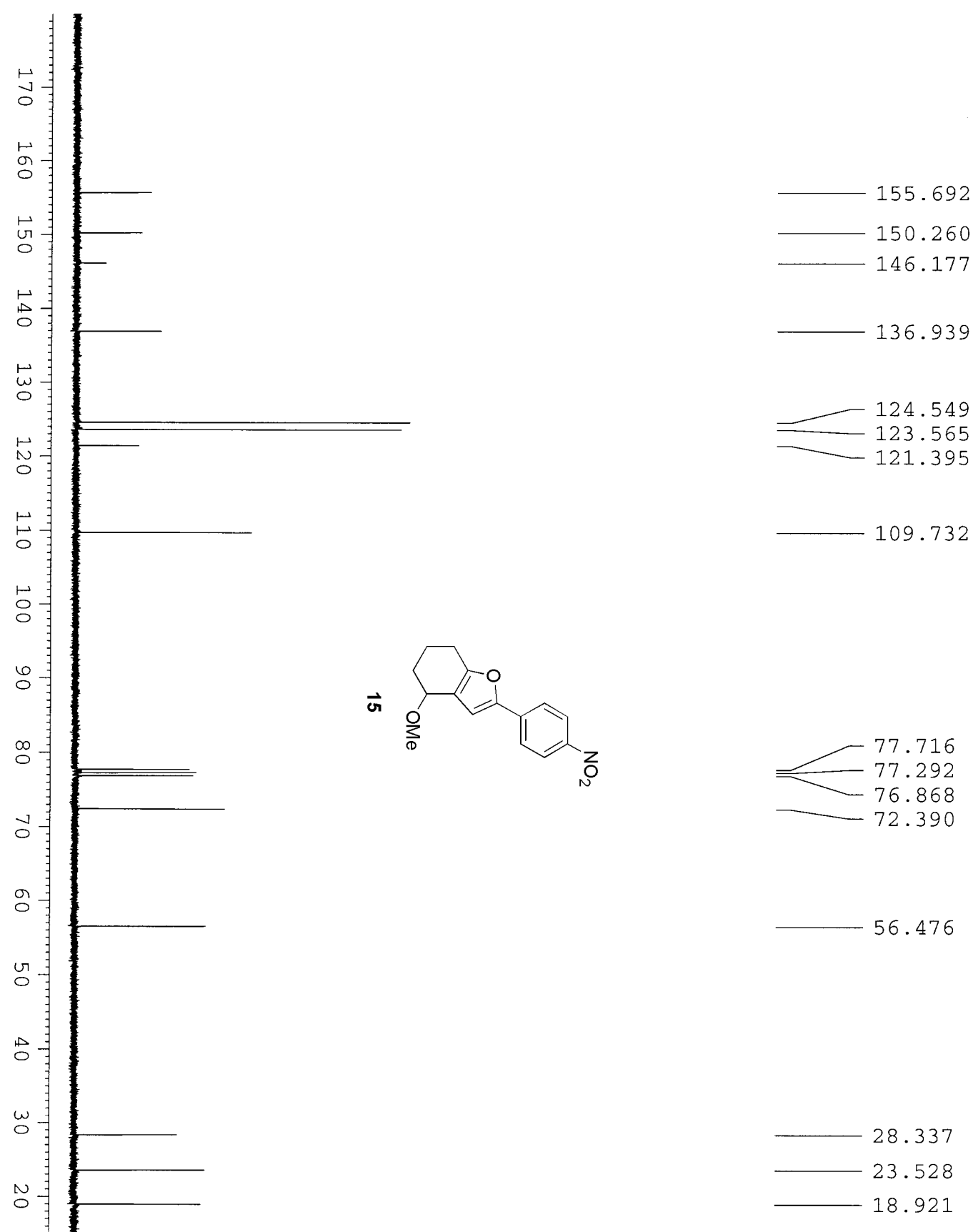



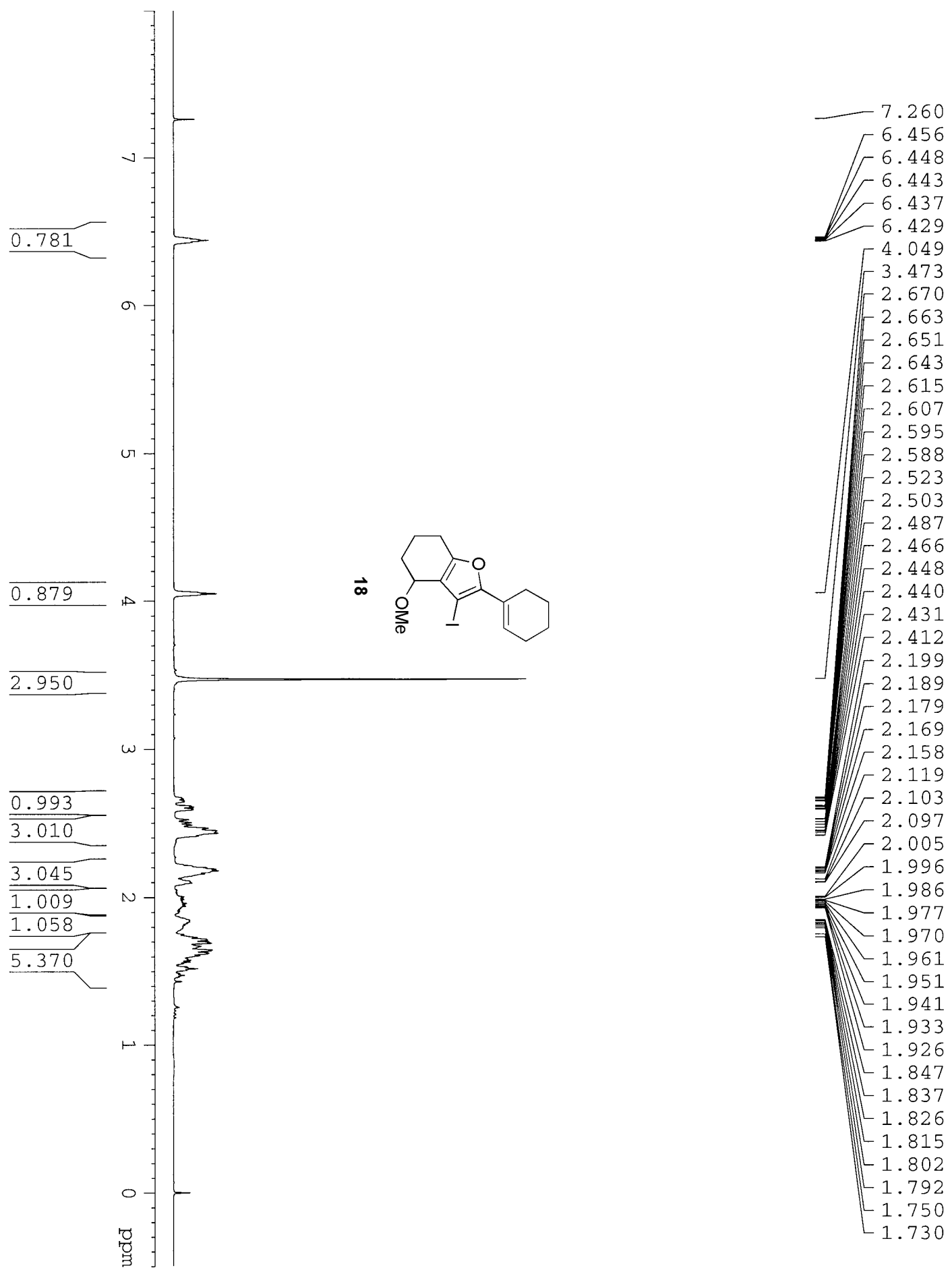

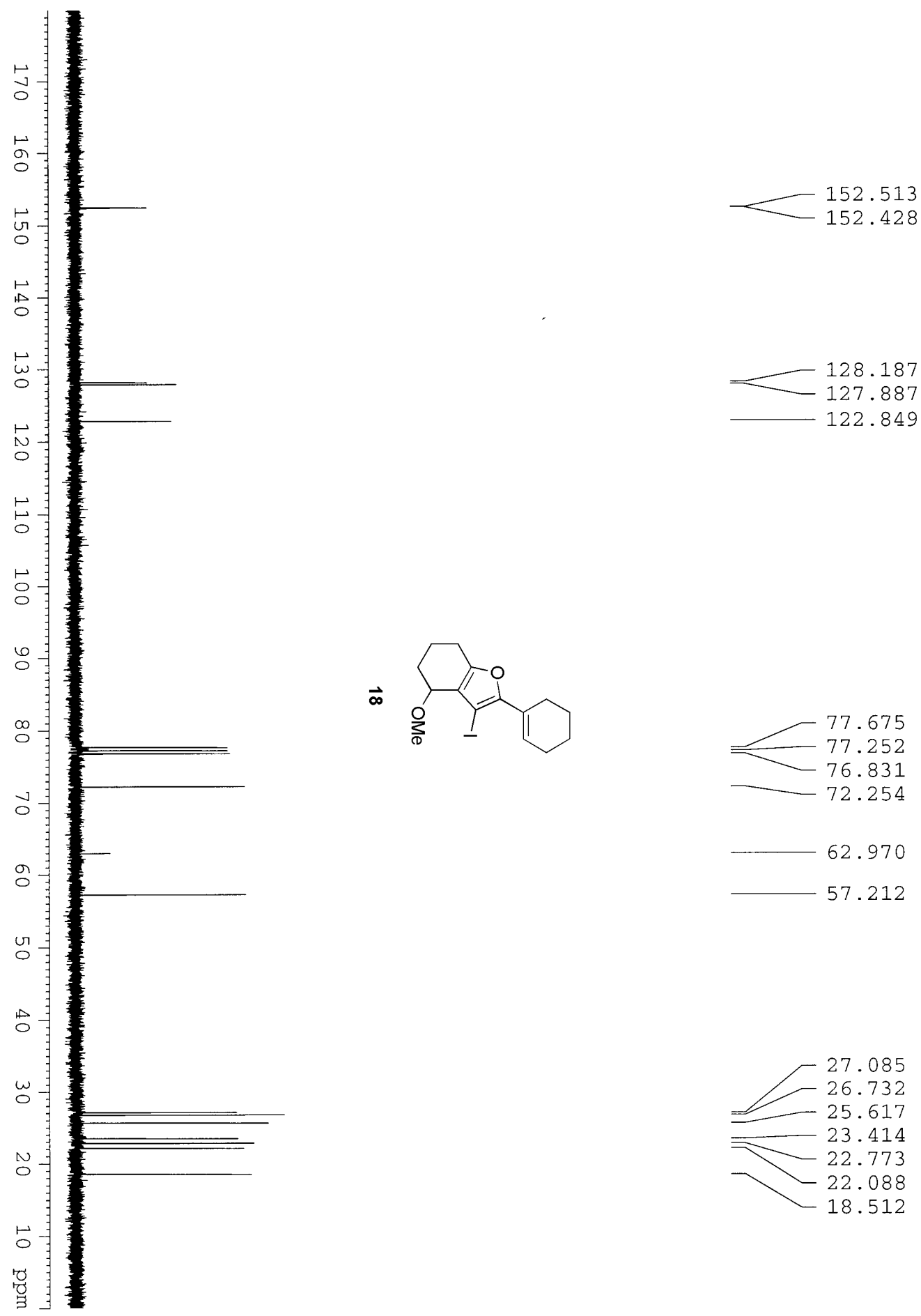


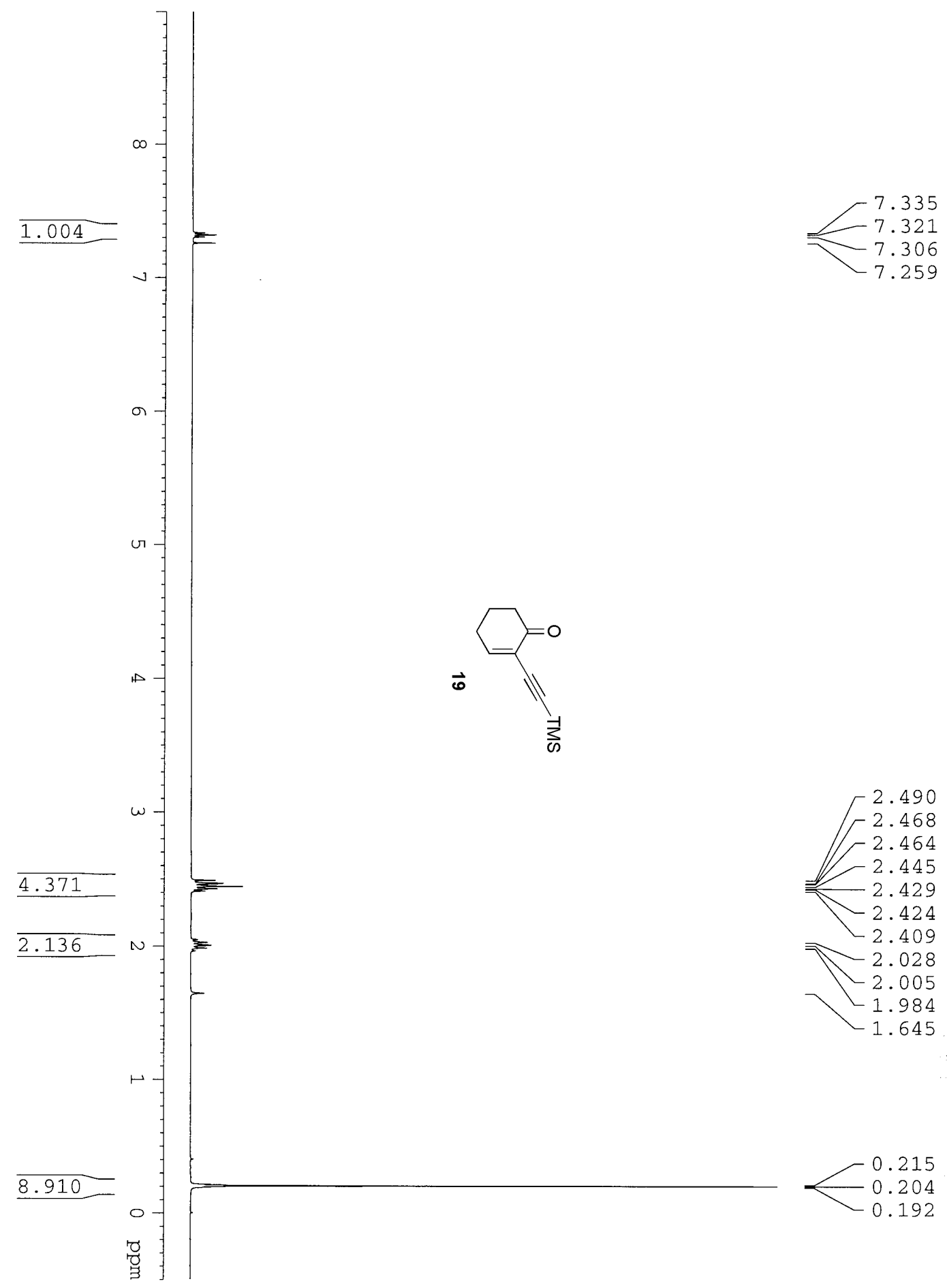




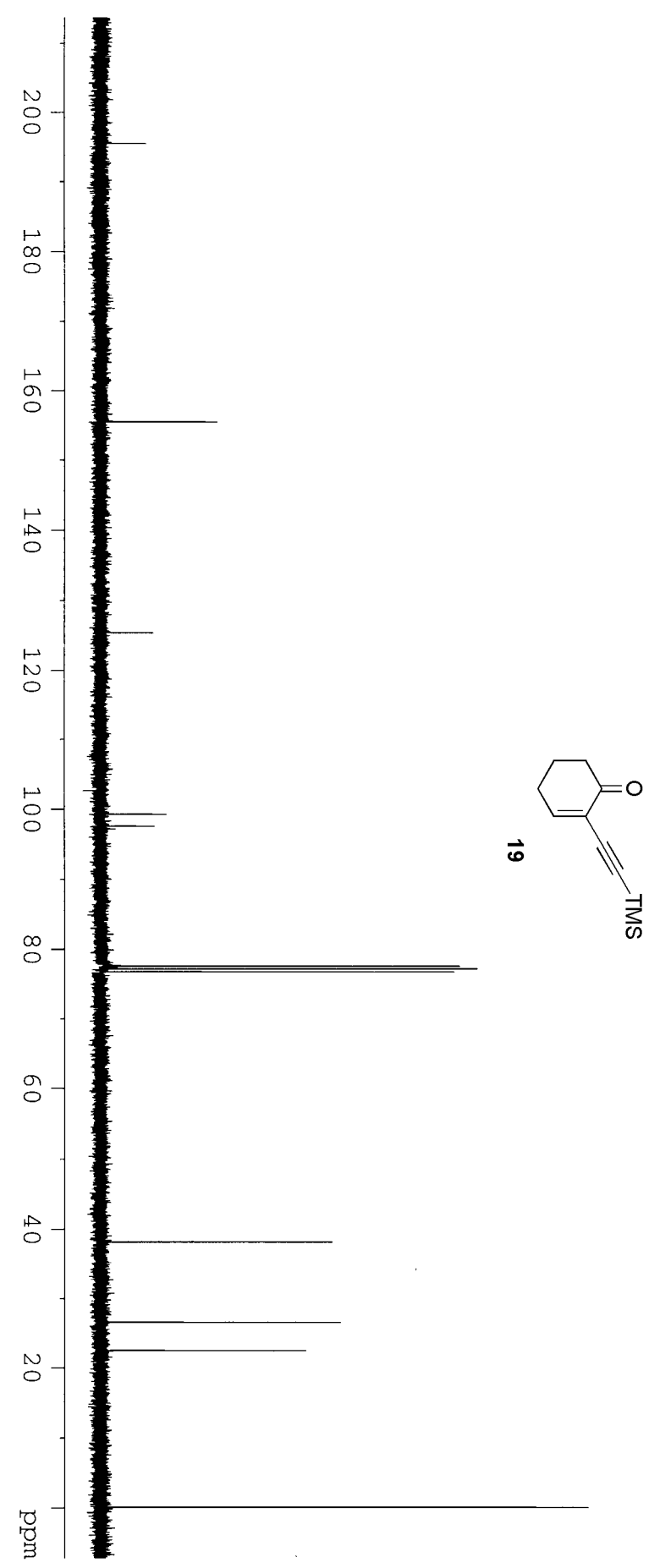

$-195.510$
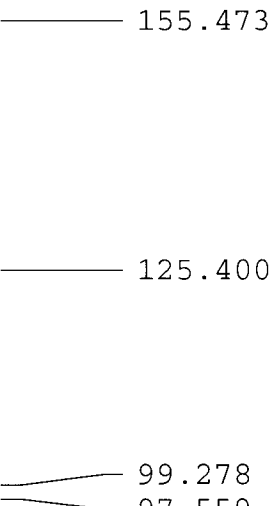

97.559

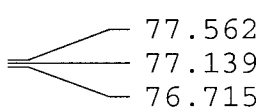

38.147

26.532

22.432

0.008 

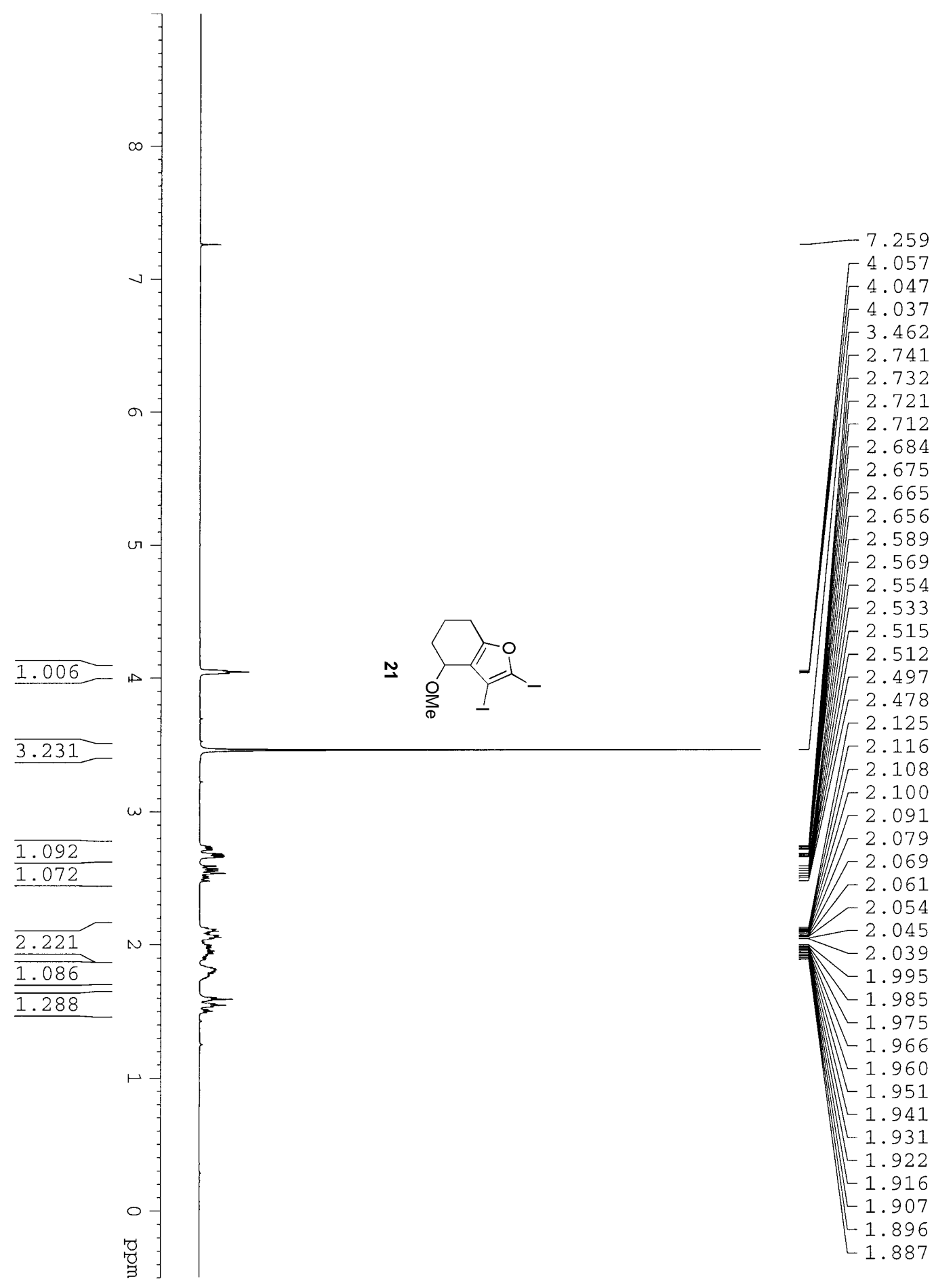


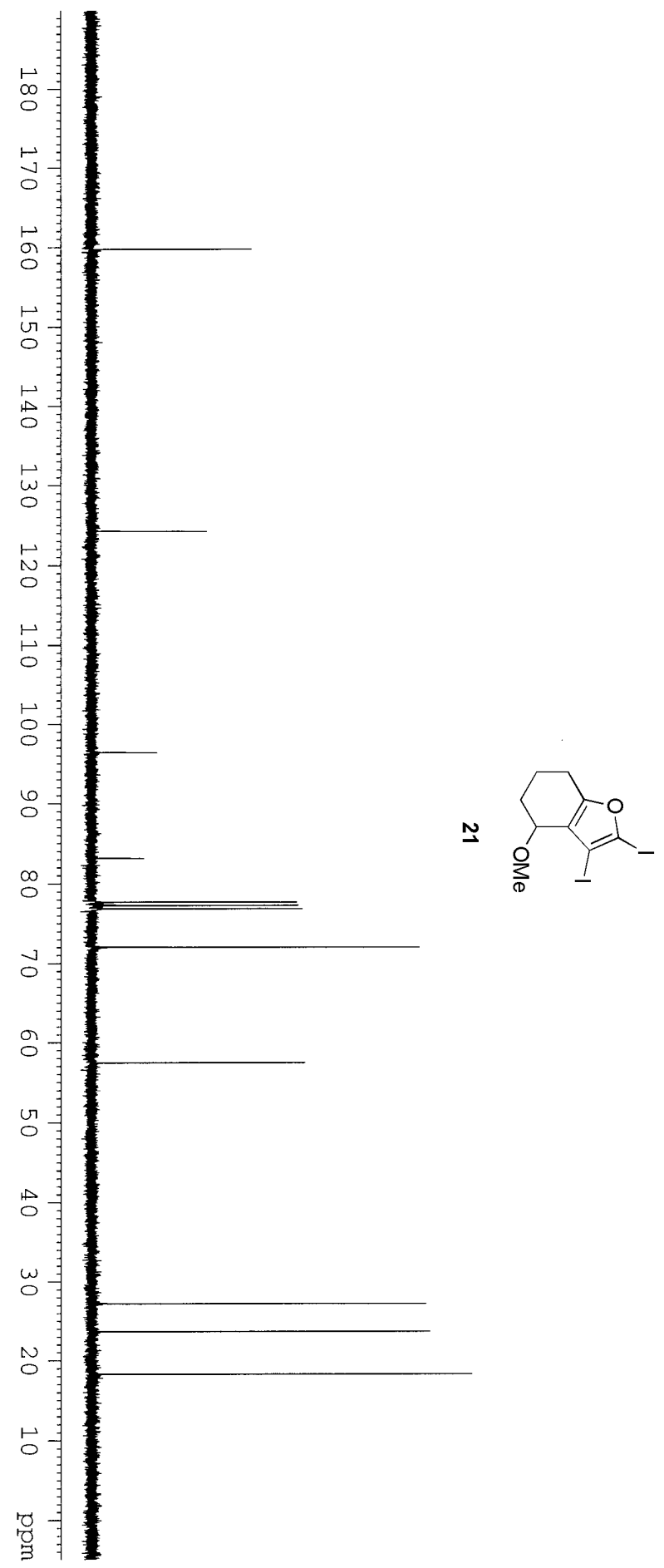

96.387

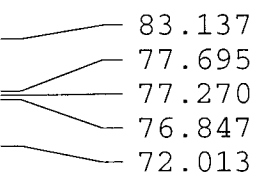

57.426

27.222

23.671

18.279 

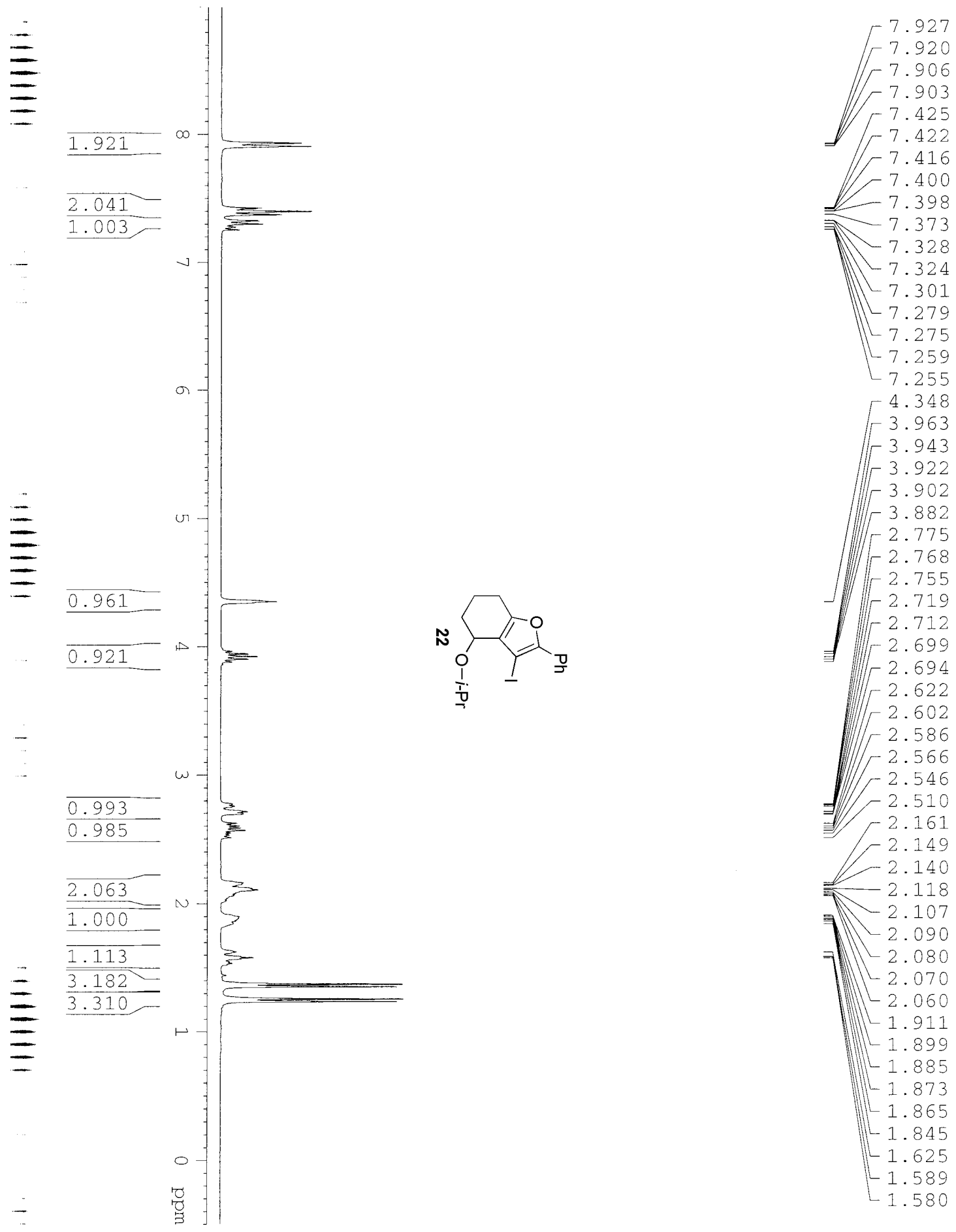


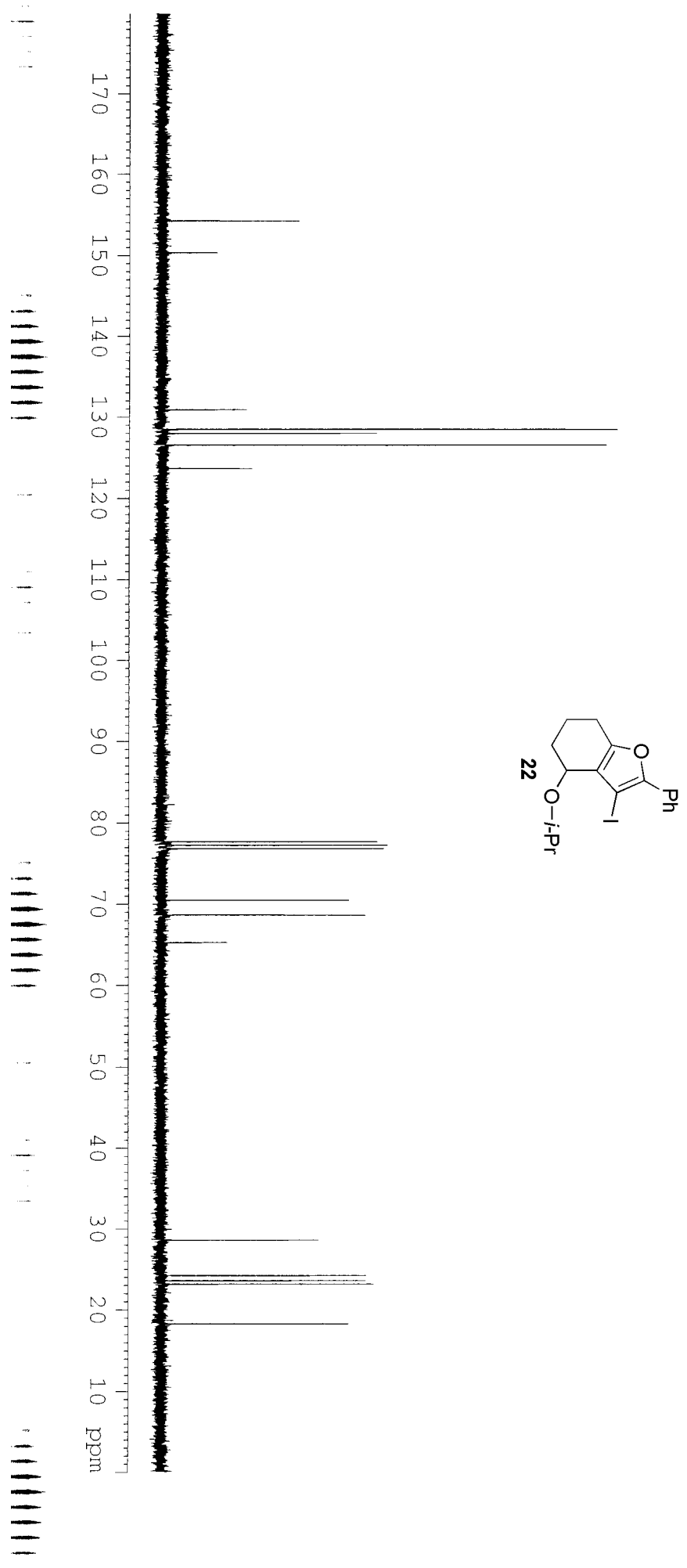



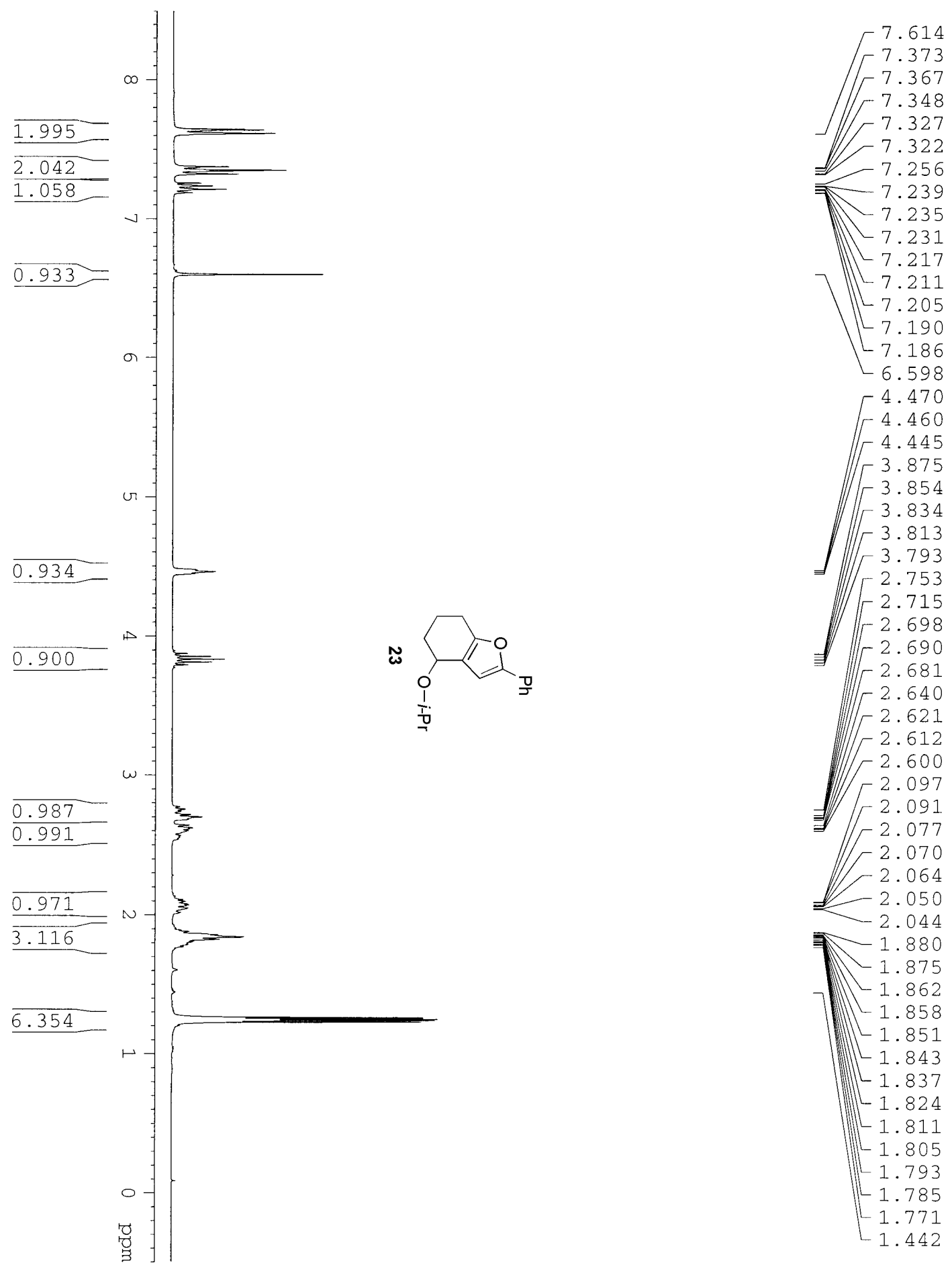

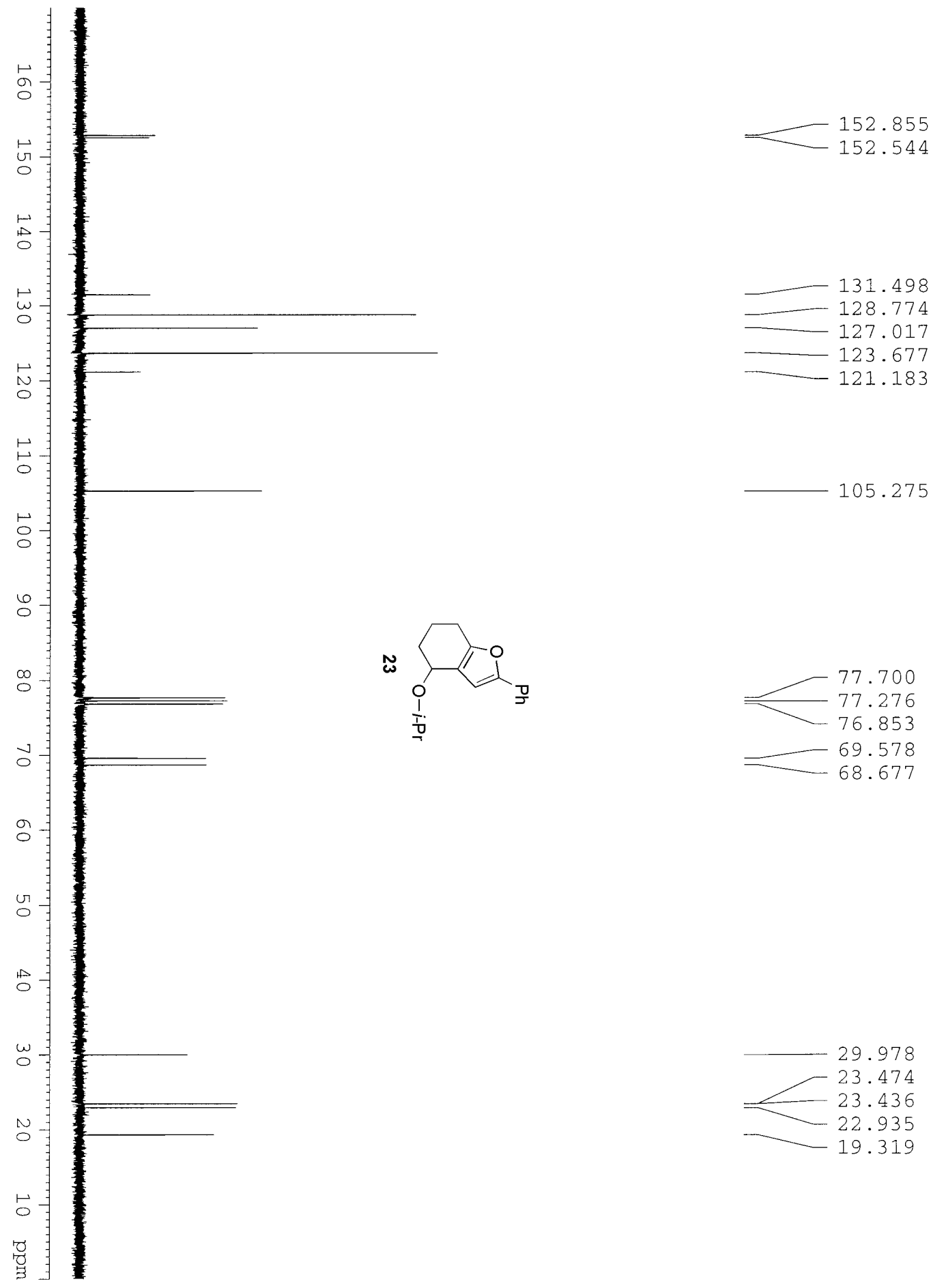

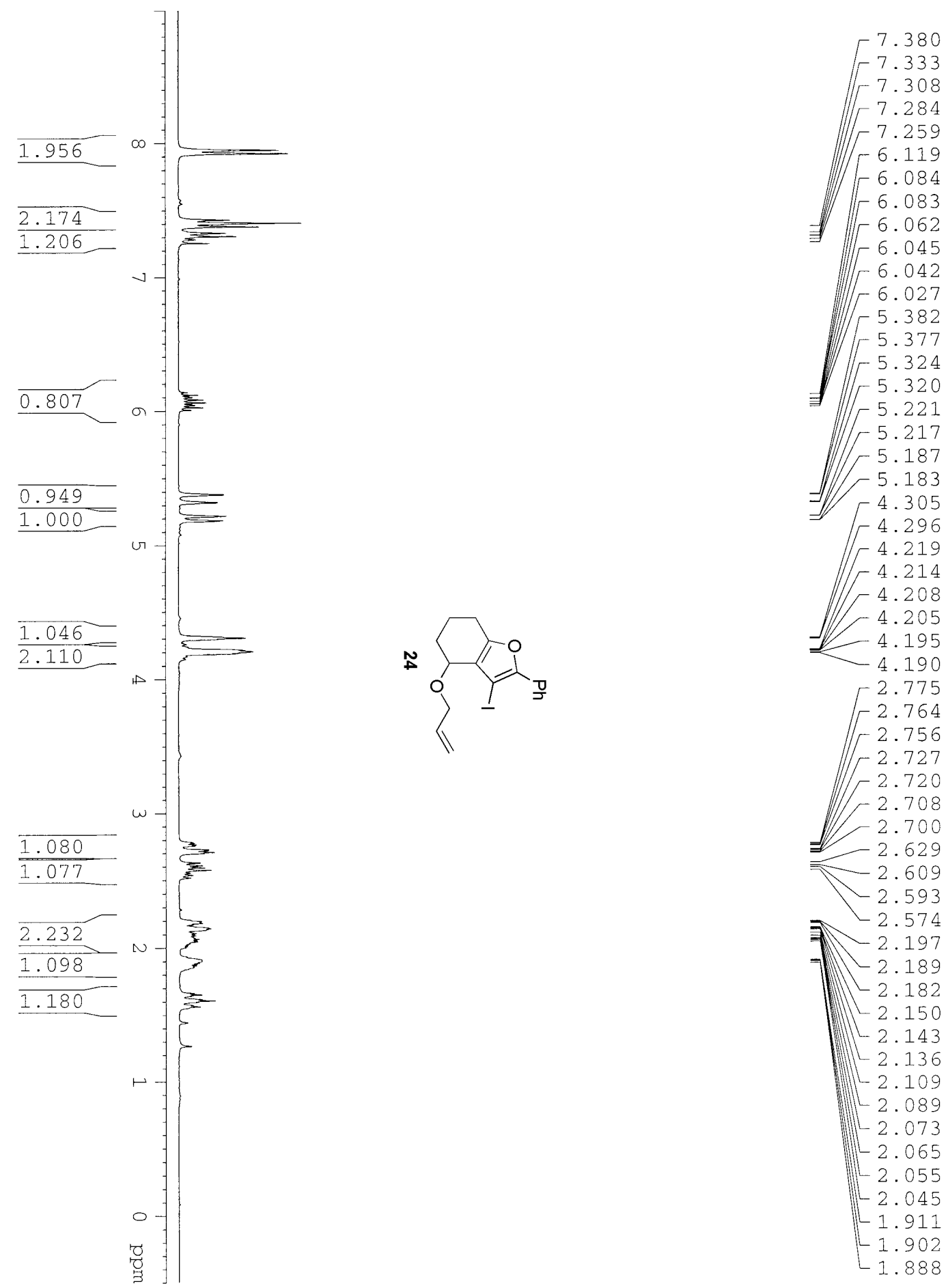


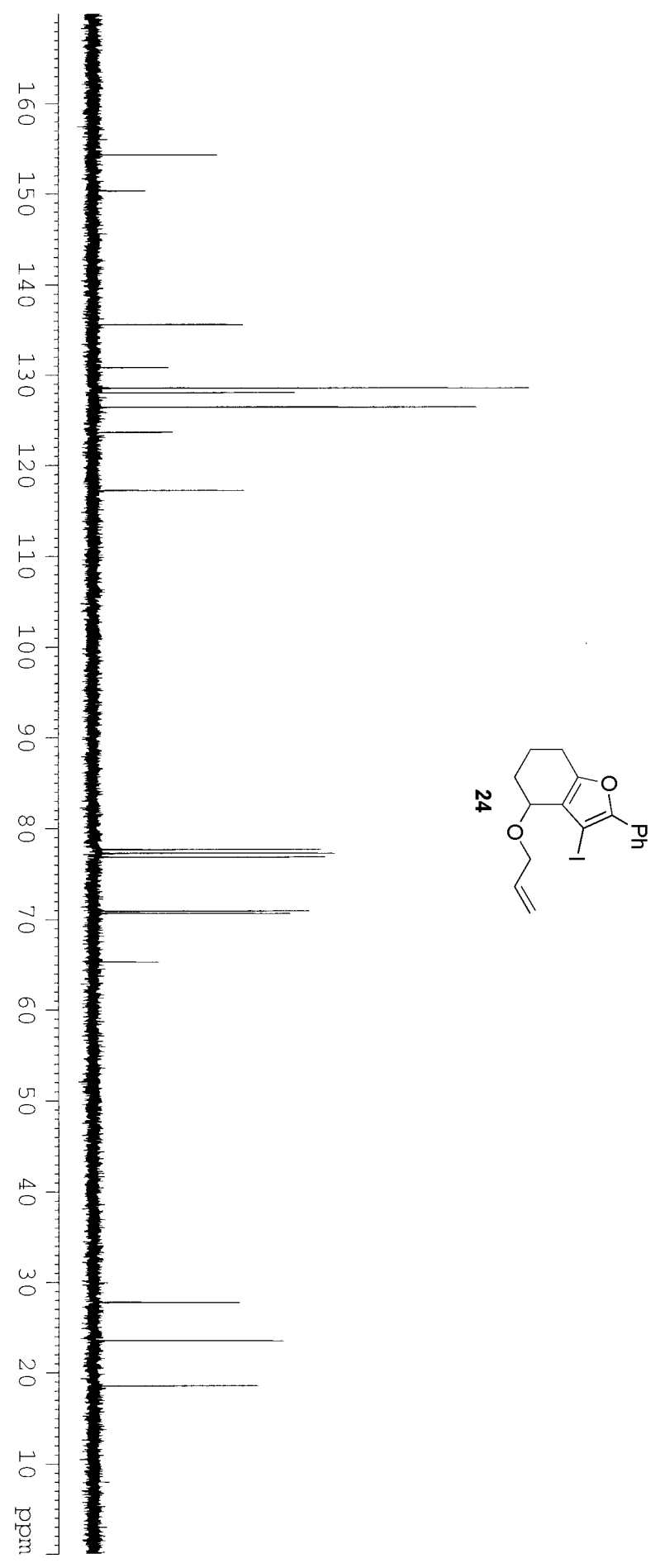

154.249 150.336
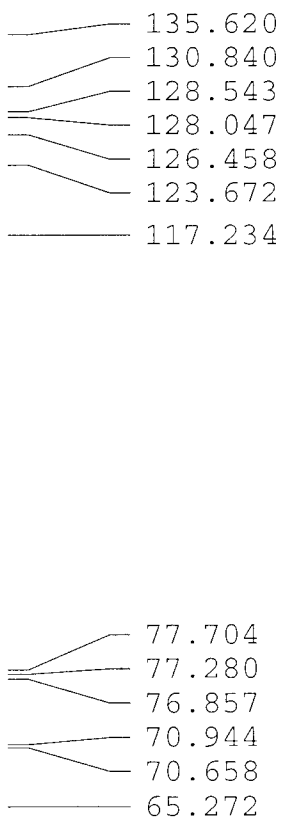

27.755

23.509

18.539 

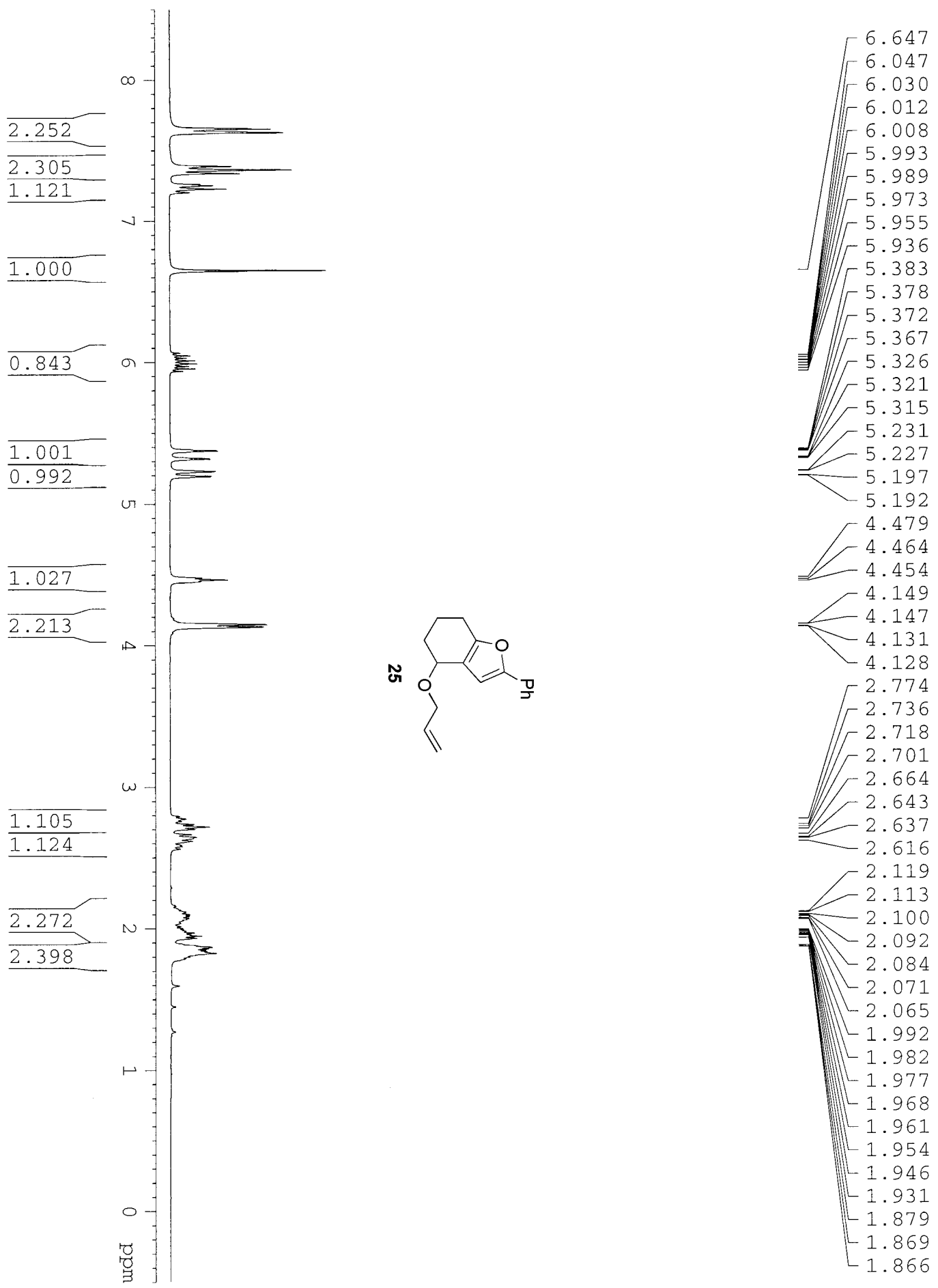

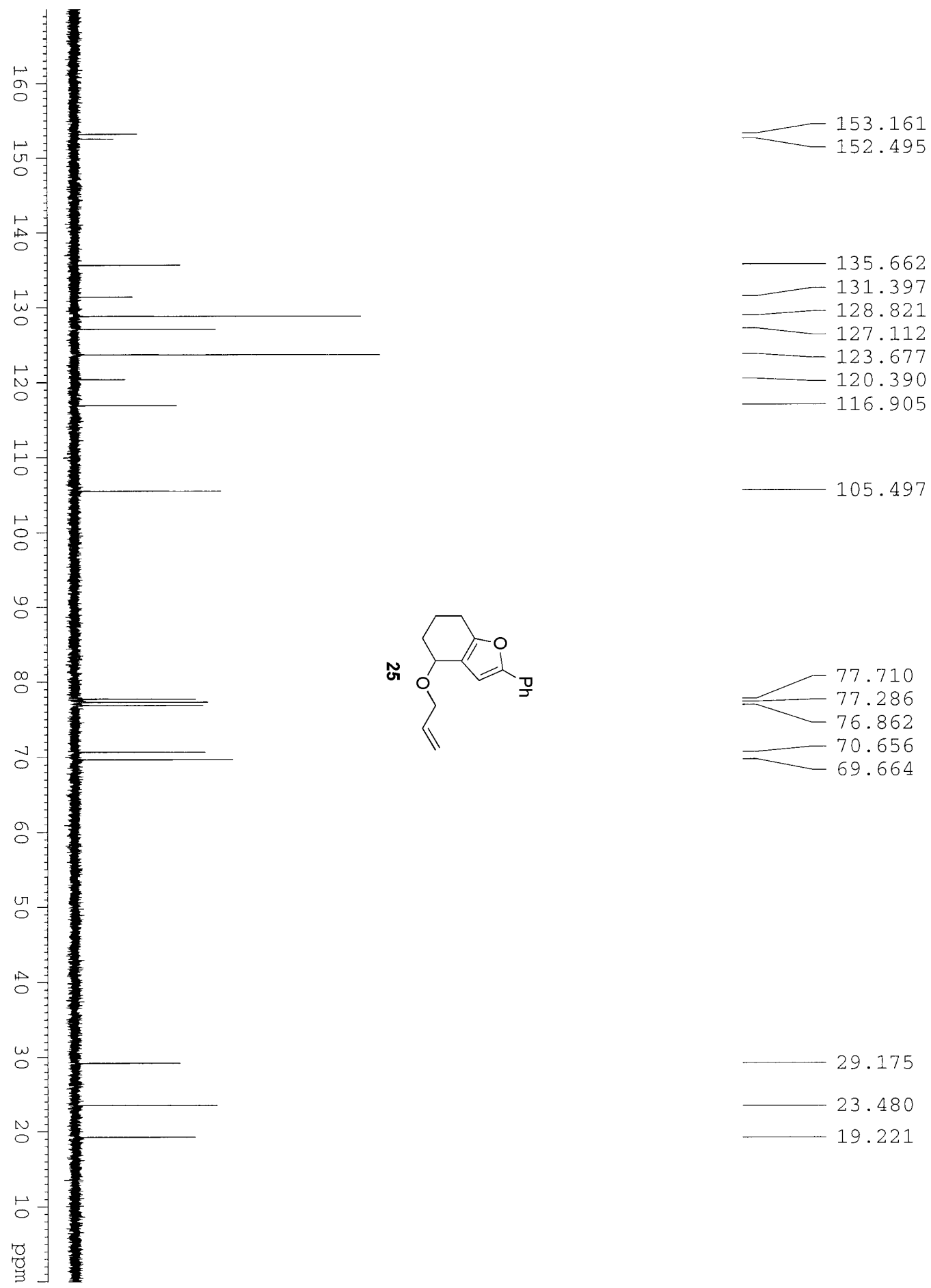

29.175

23.480

19.221 

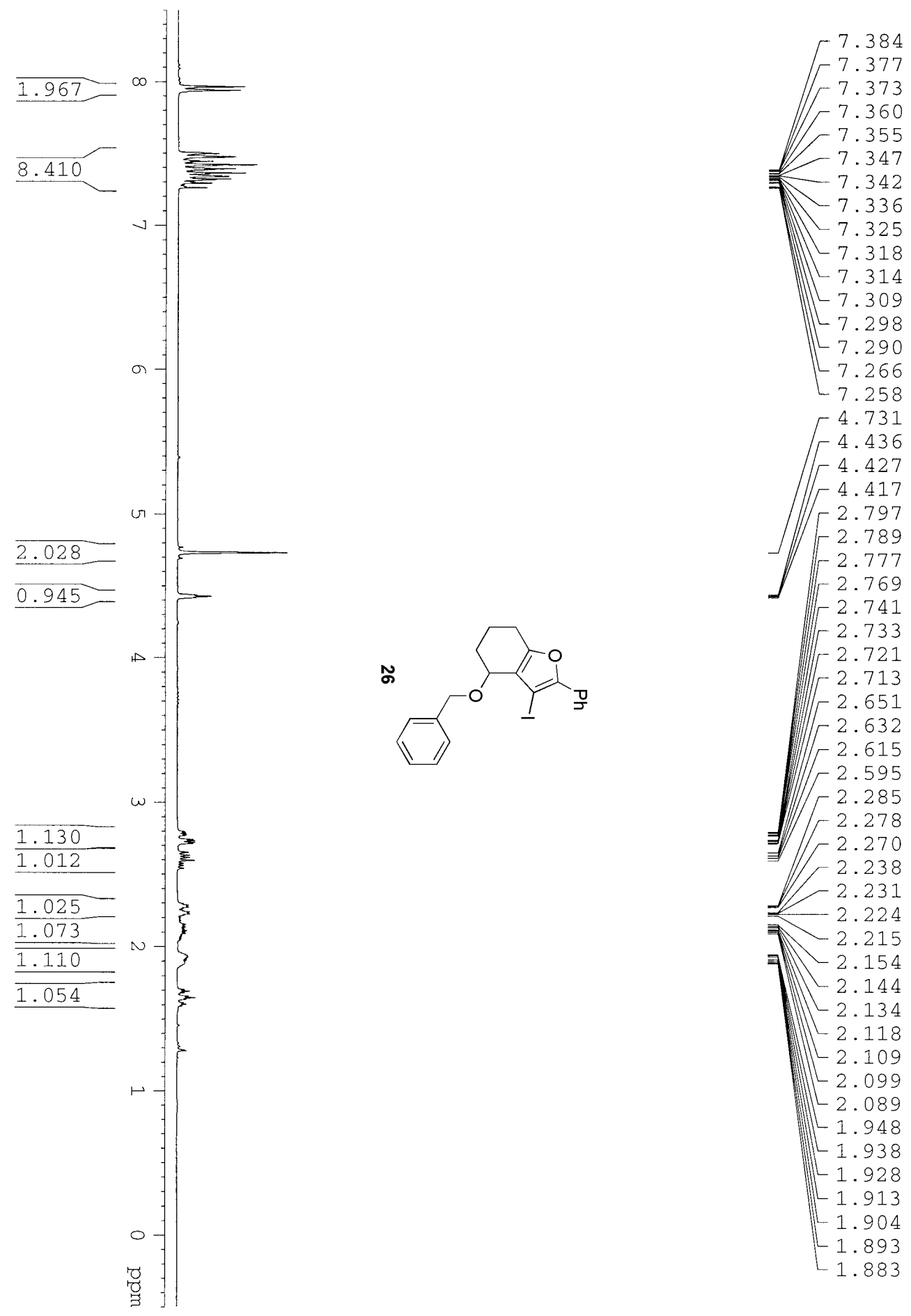

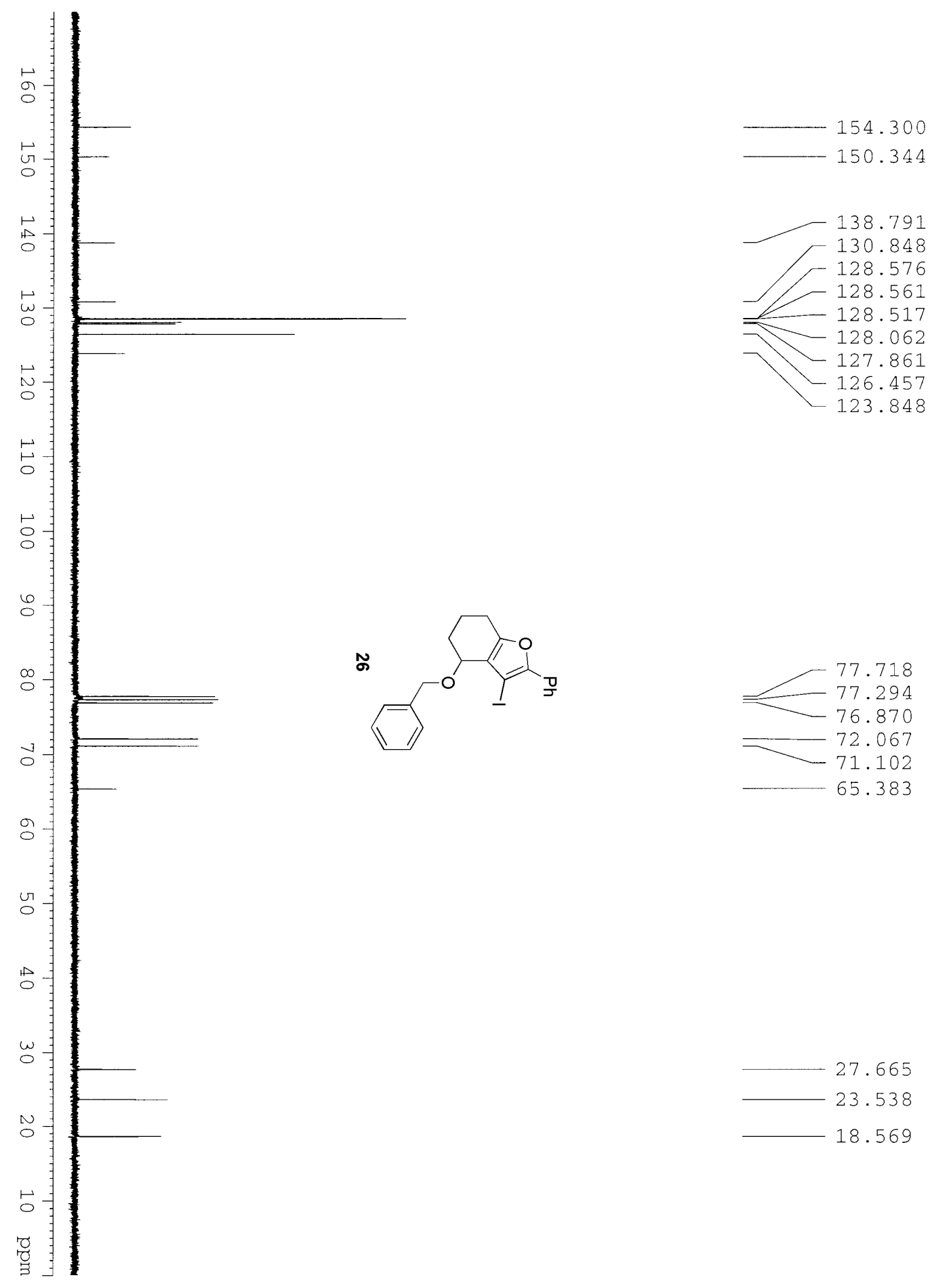

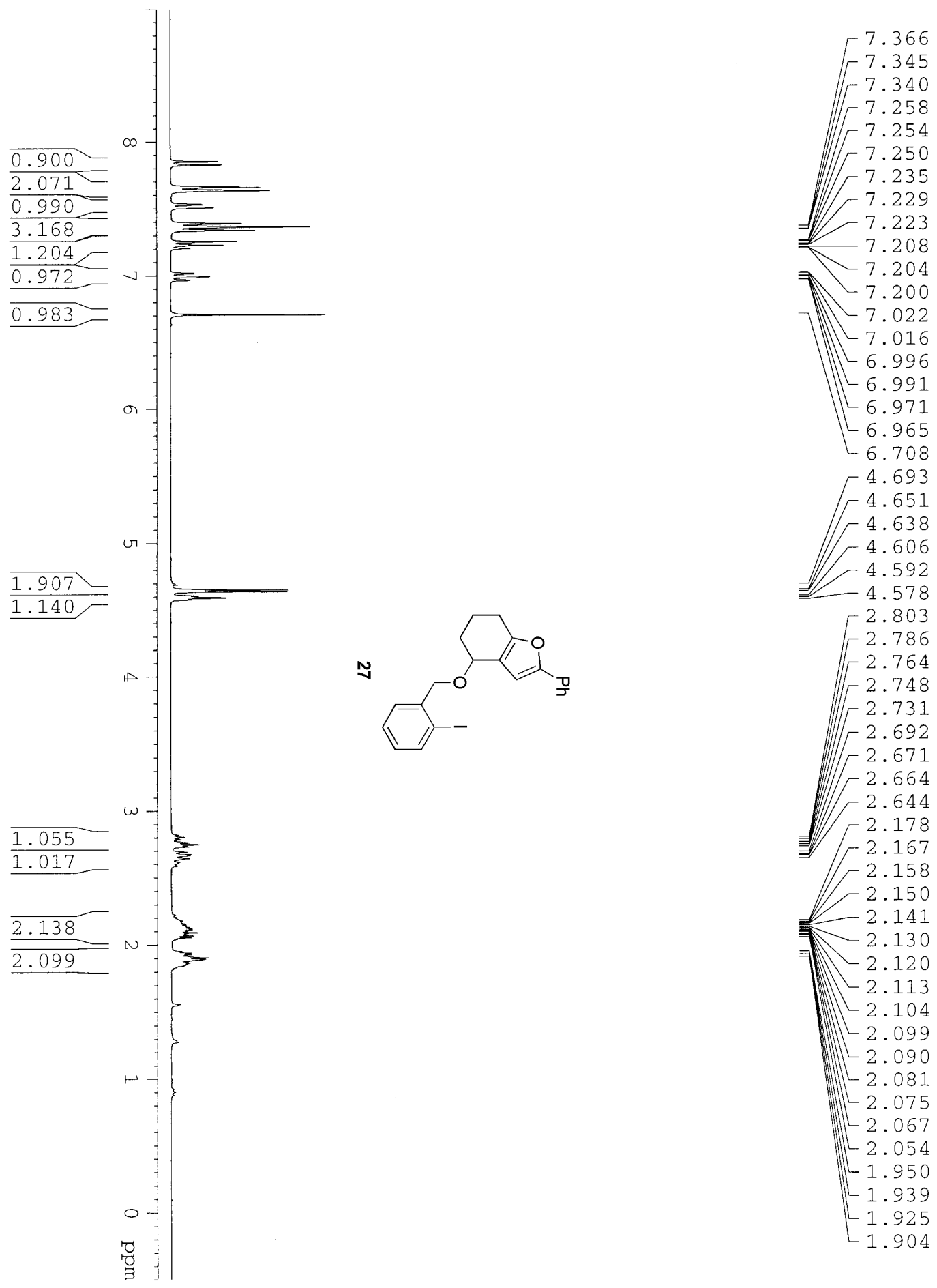

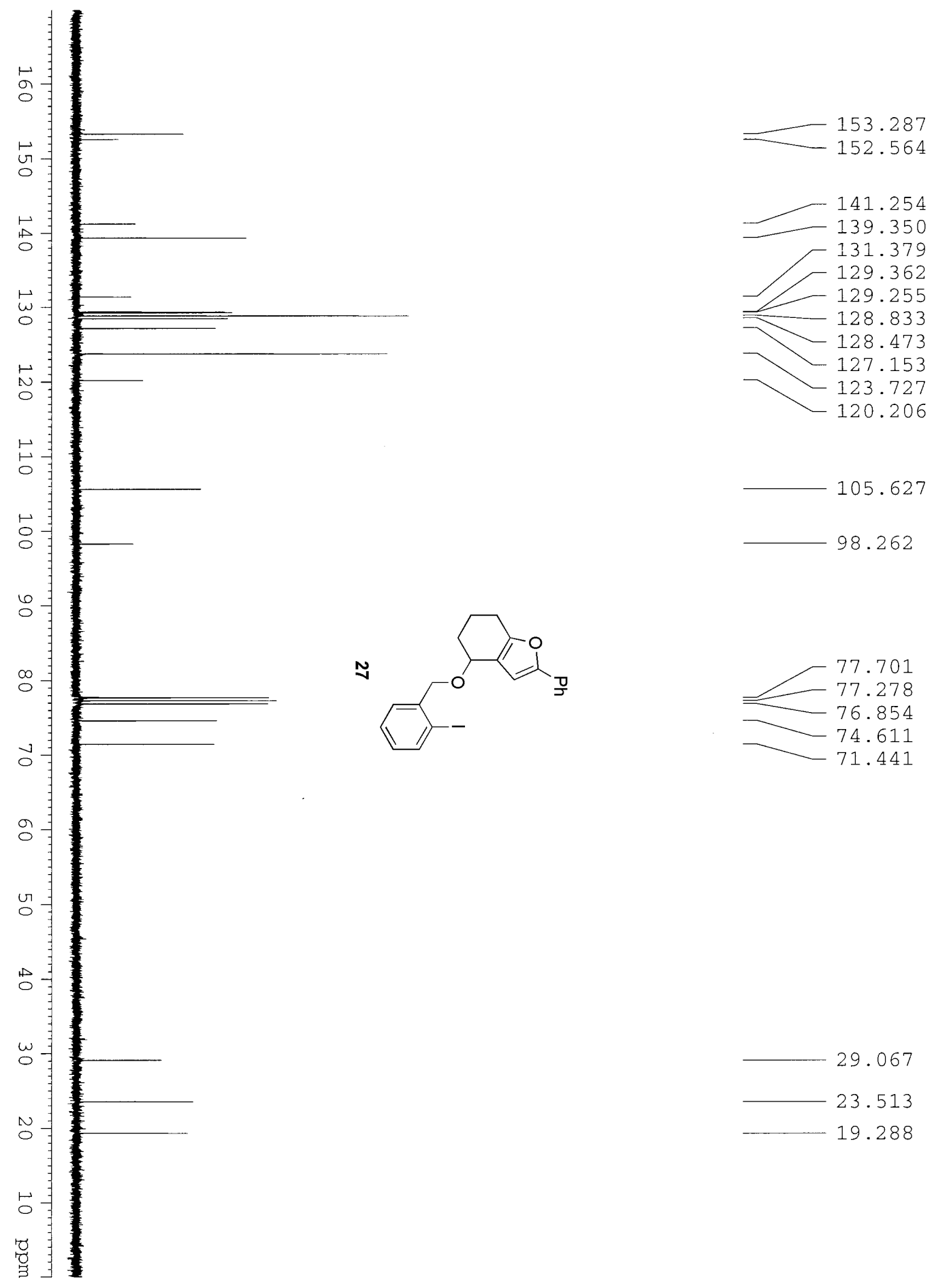

29.067

23.513 19.288 

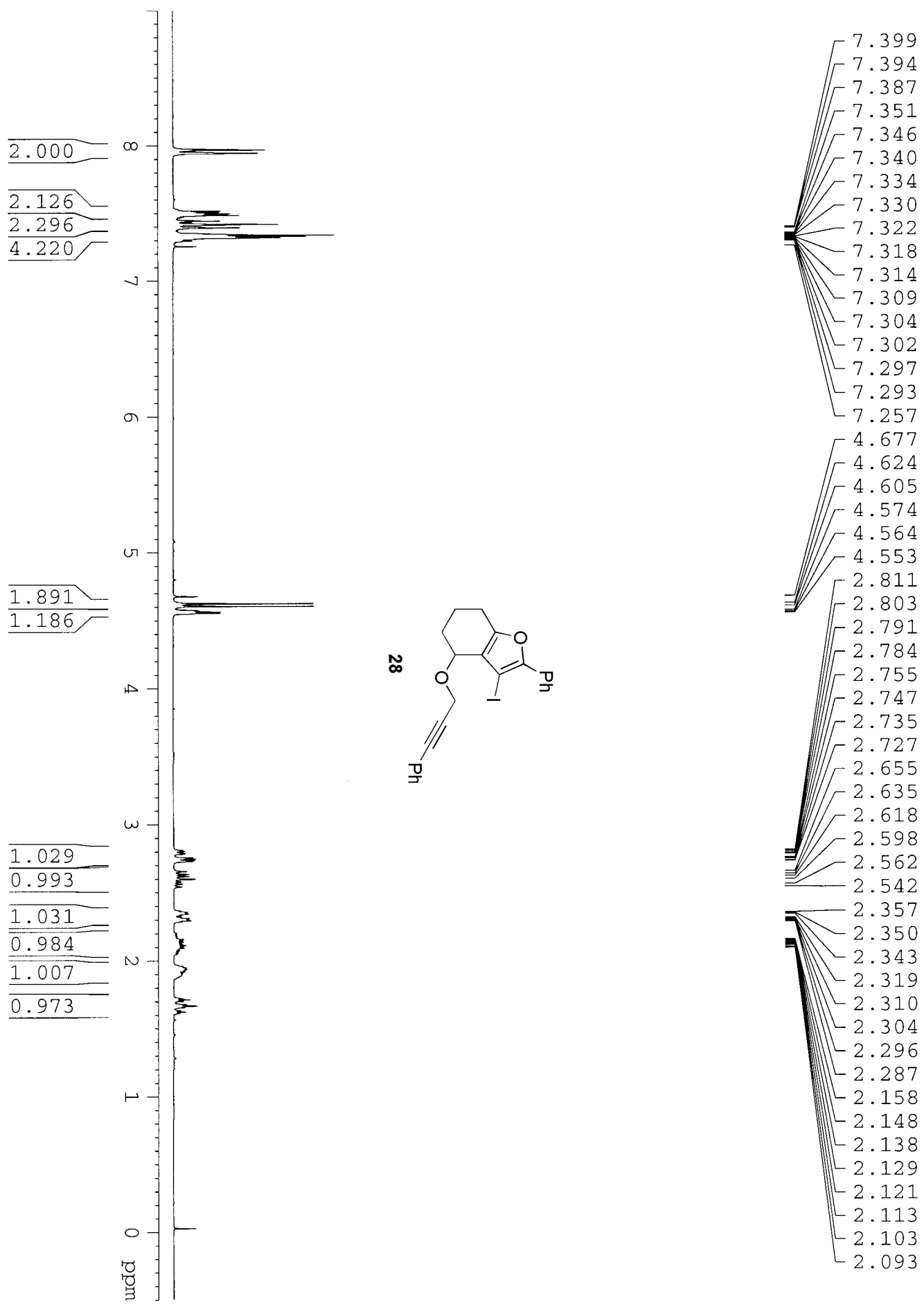

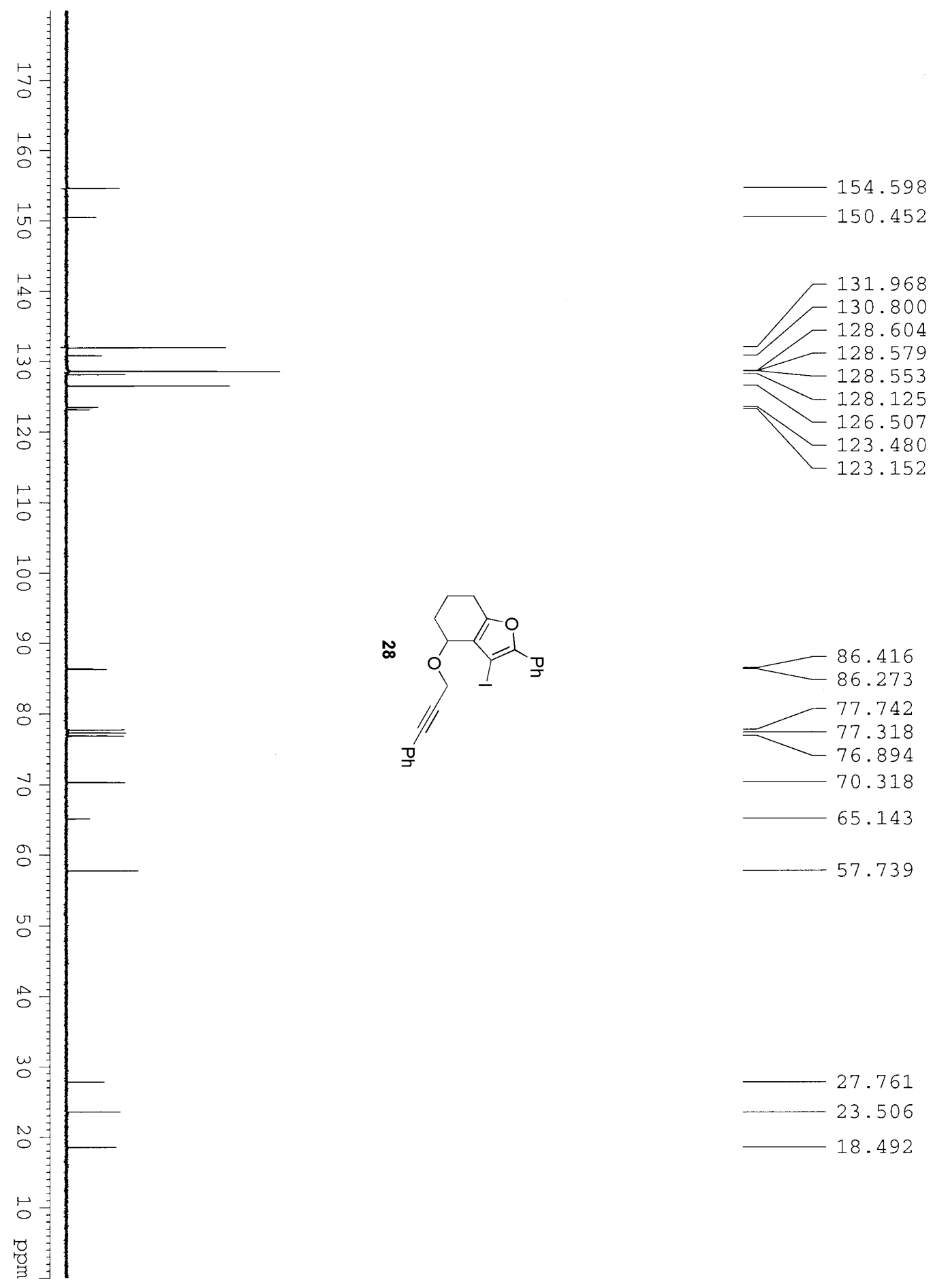

23.506 18.492 

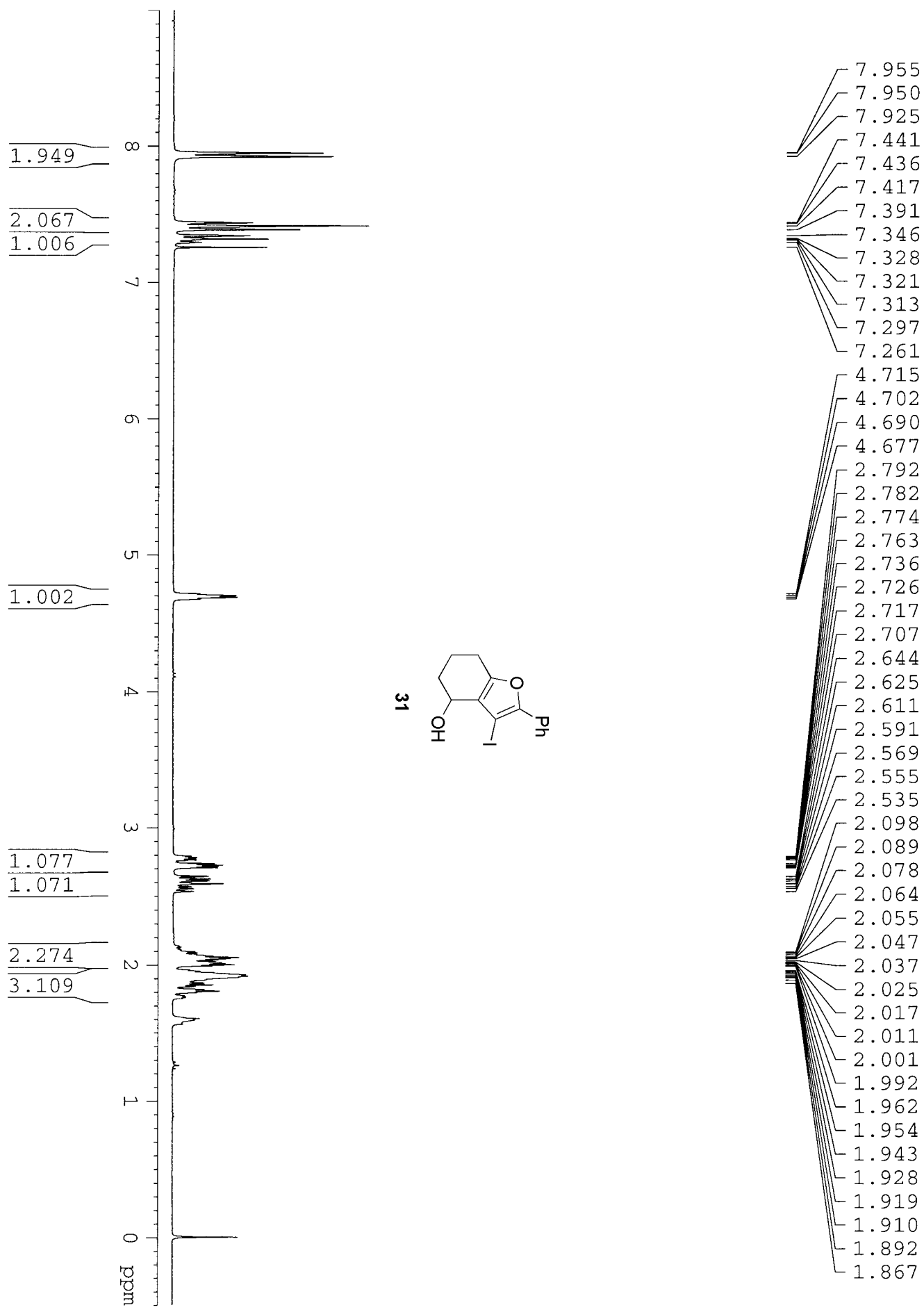

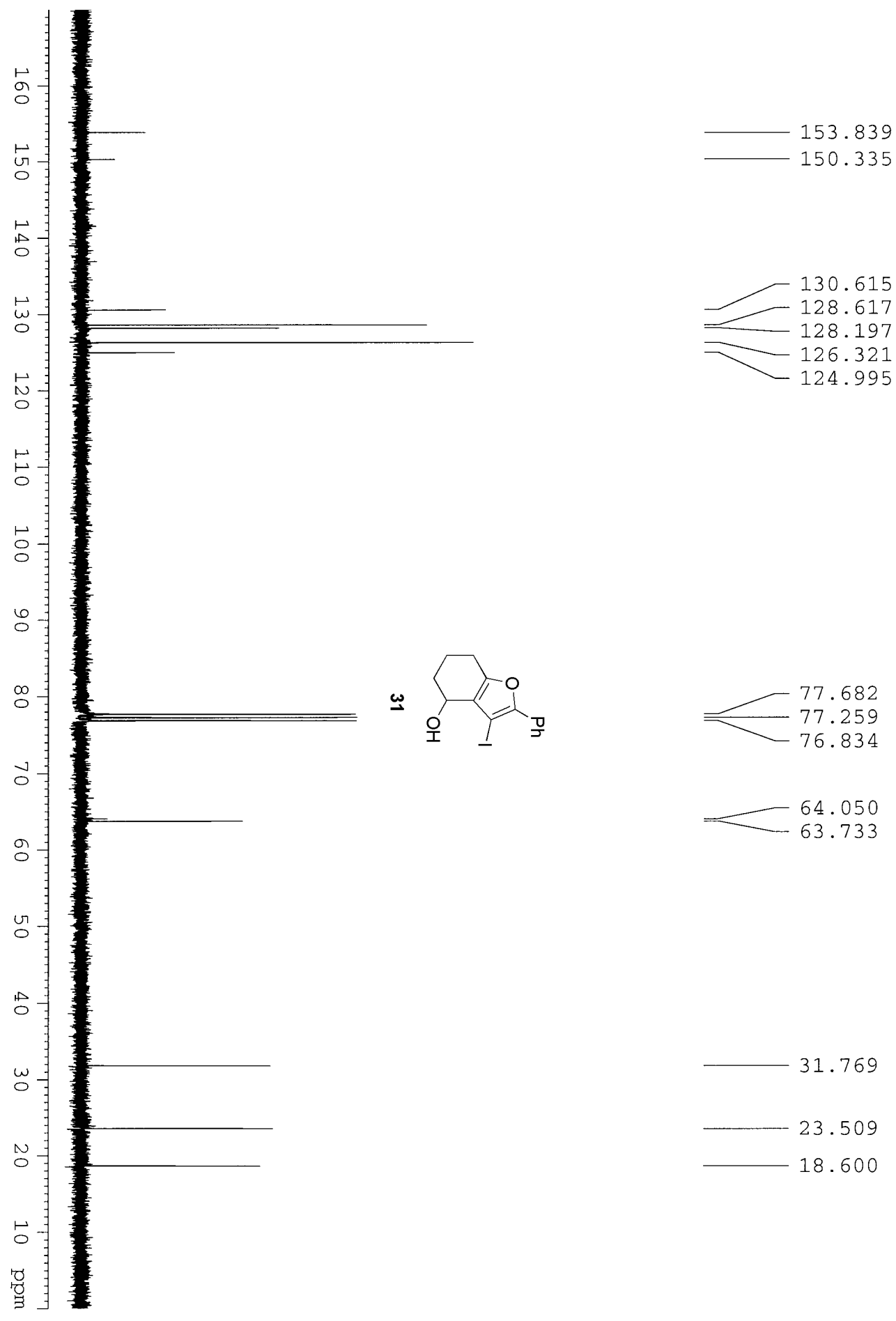

63.733

31.769

23.509

18.600 


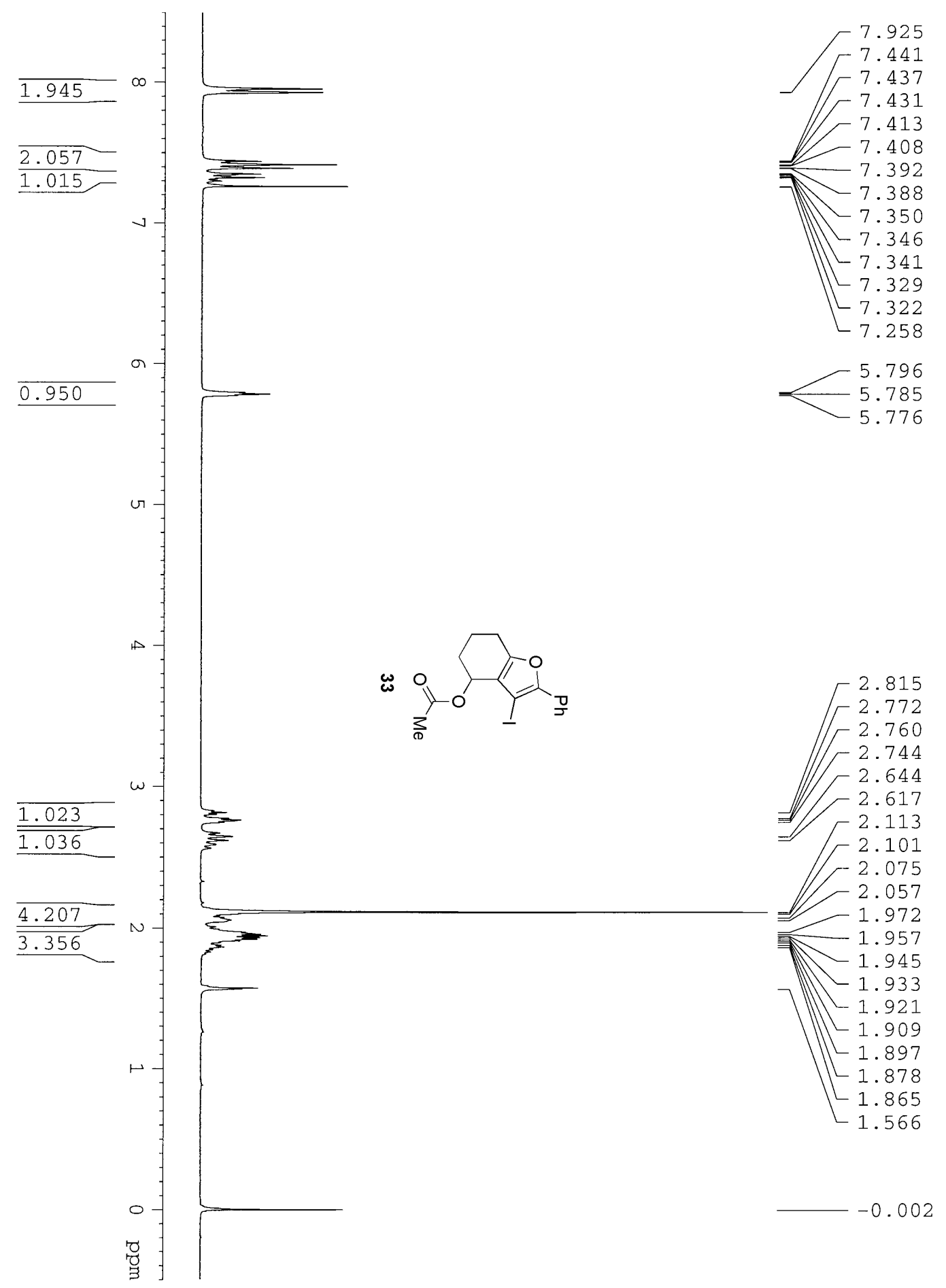



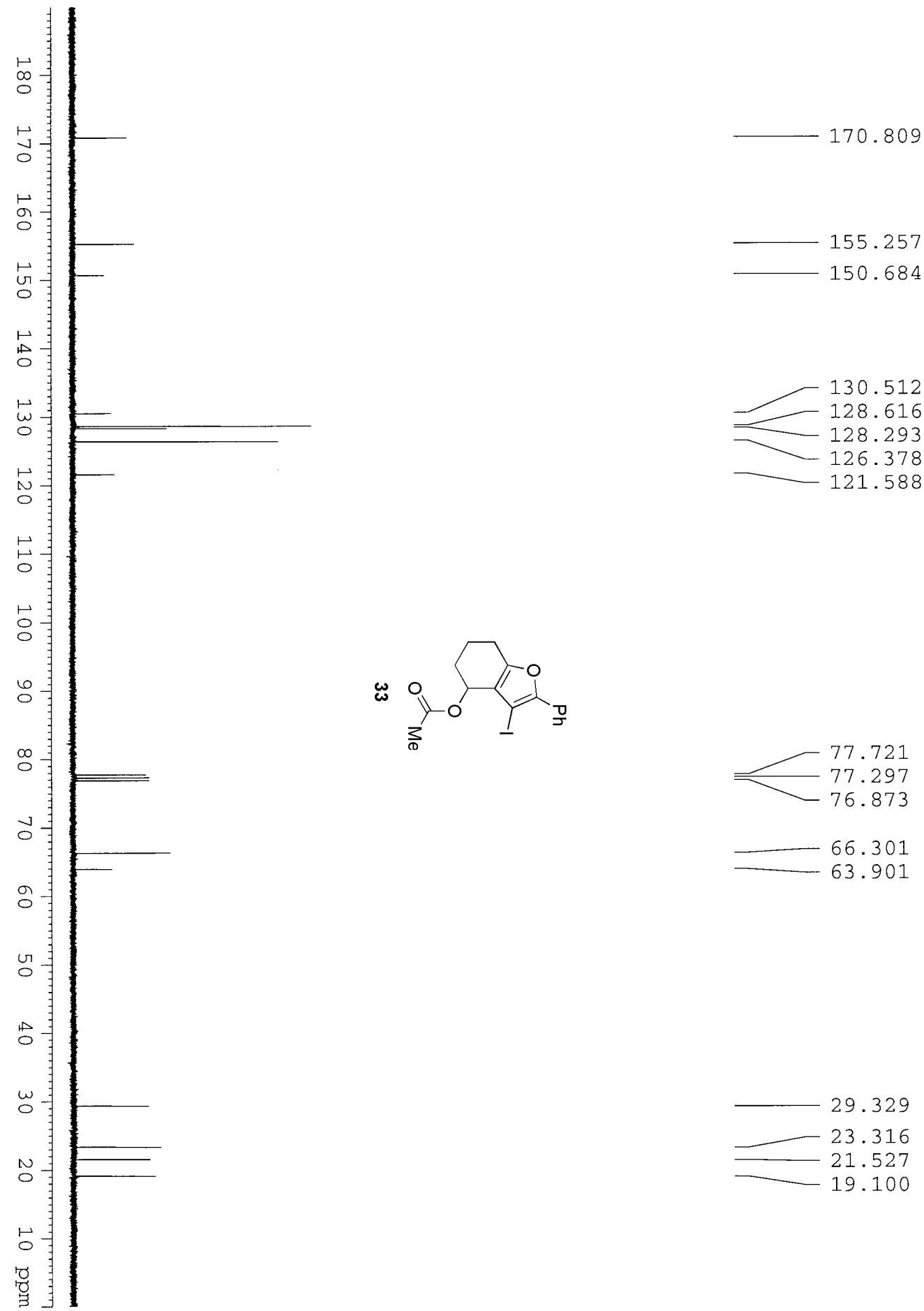

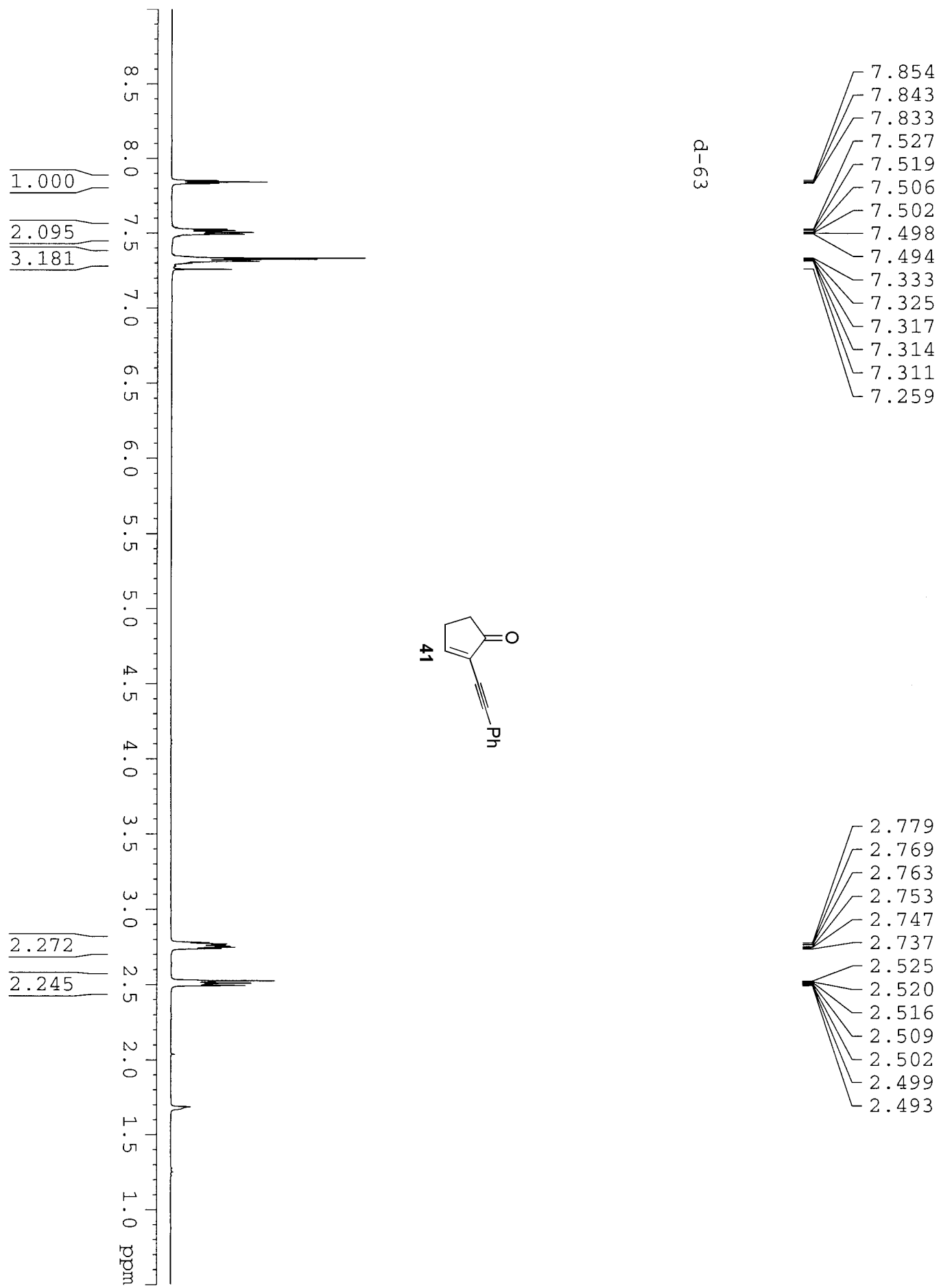

$-2.525$

2.520

$-2.516$

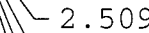

$-2.502$

$-2.499$

$-2.493$ 

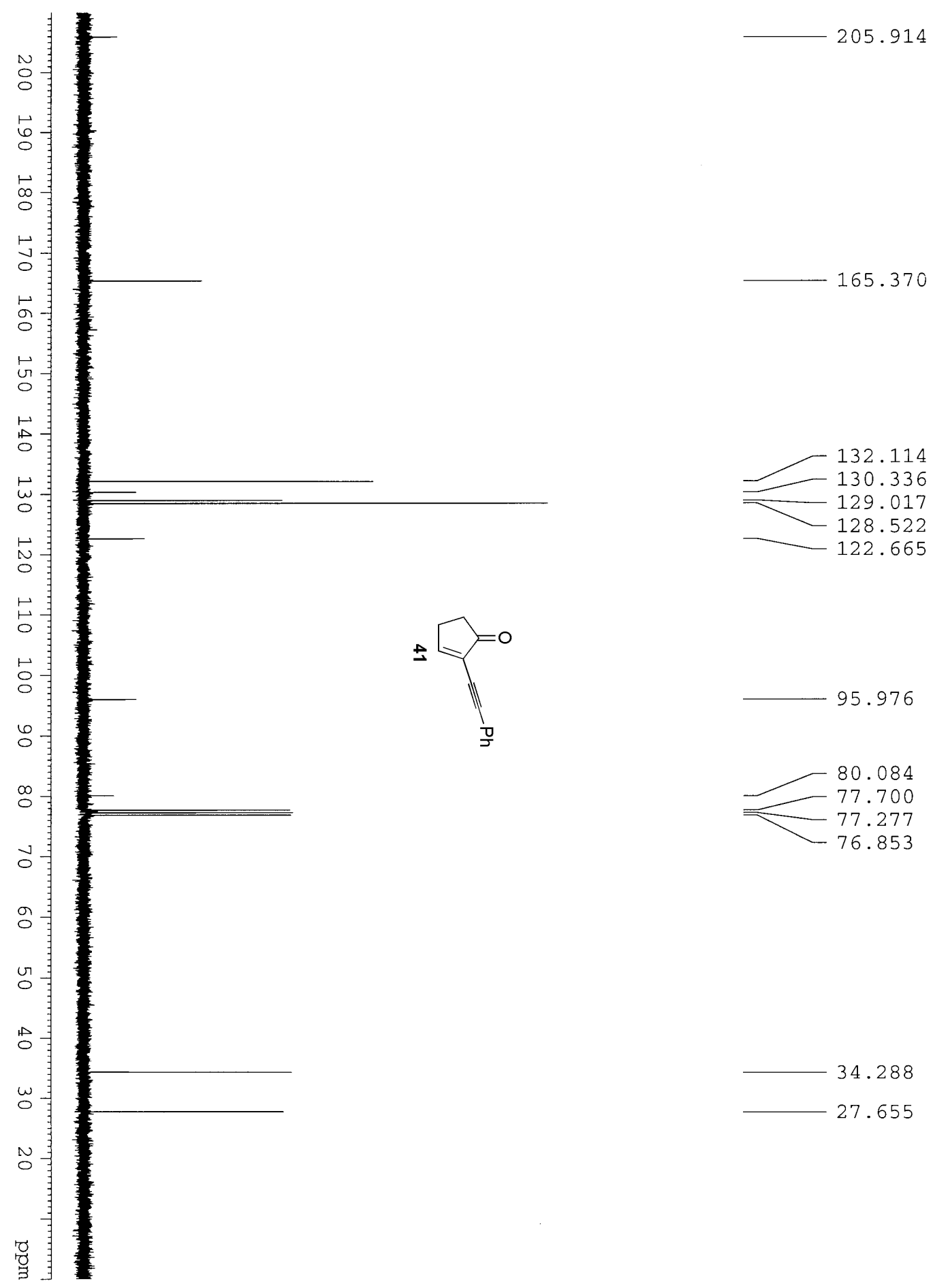

95.976

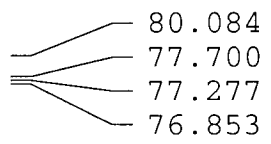

34.288

27.655 

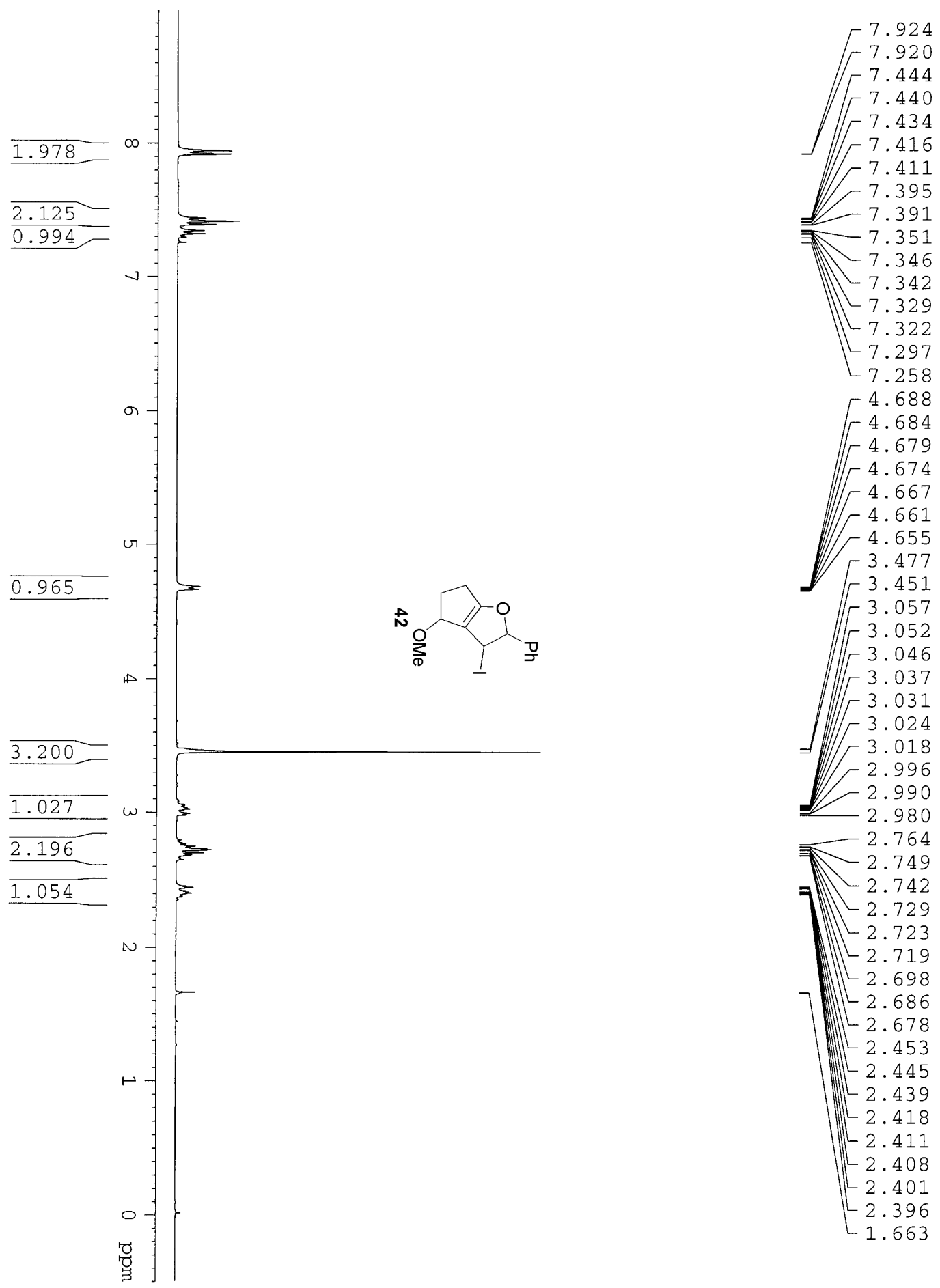

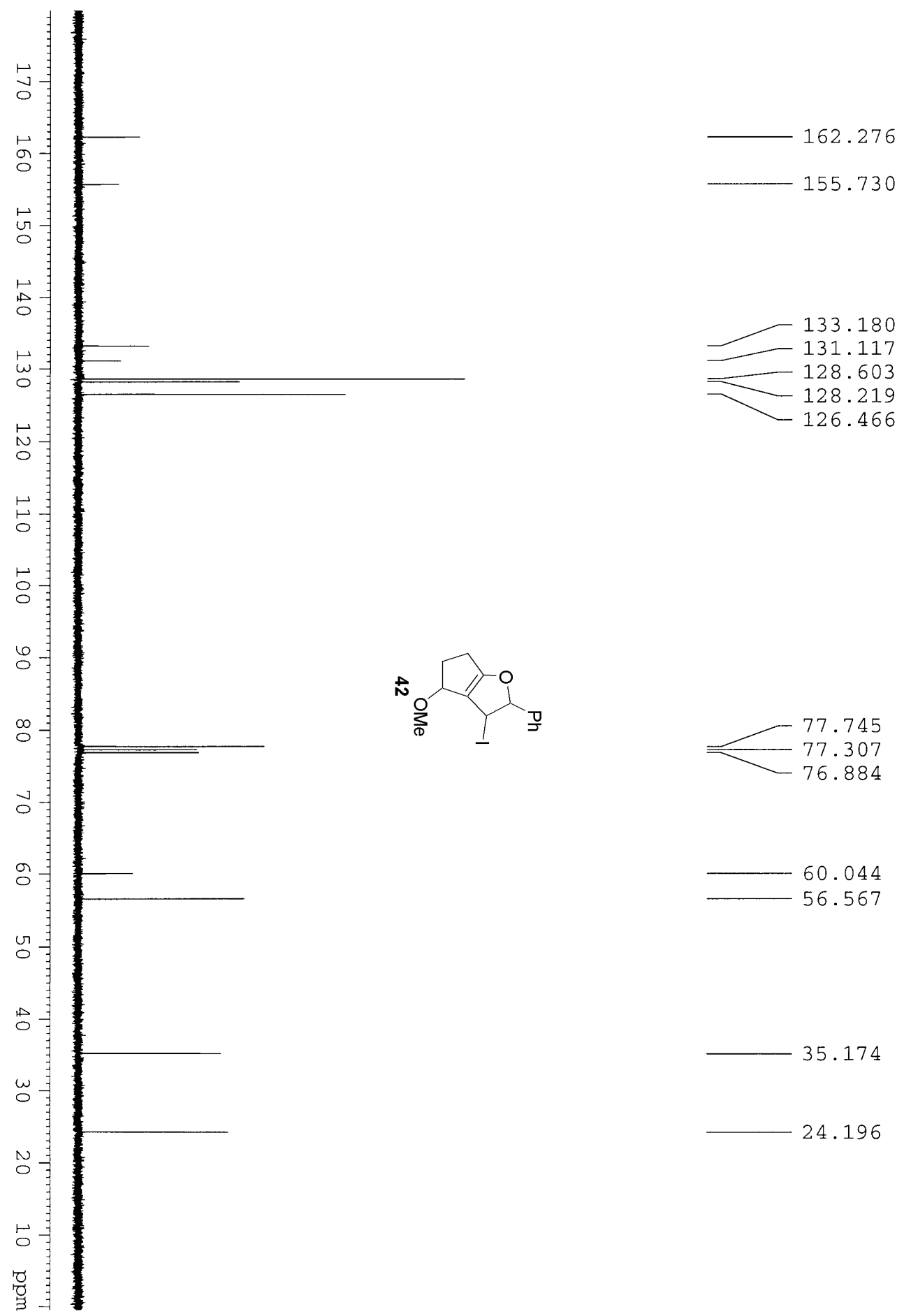

$-60.044$ 56.567

35.174

24.196 

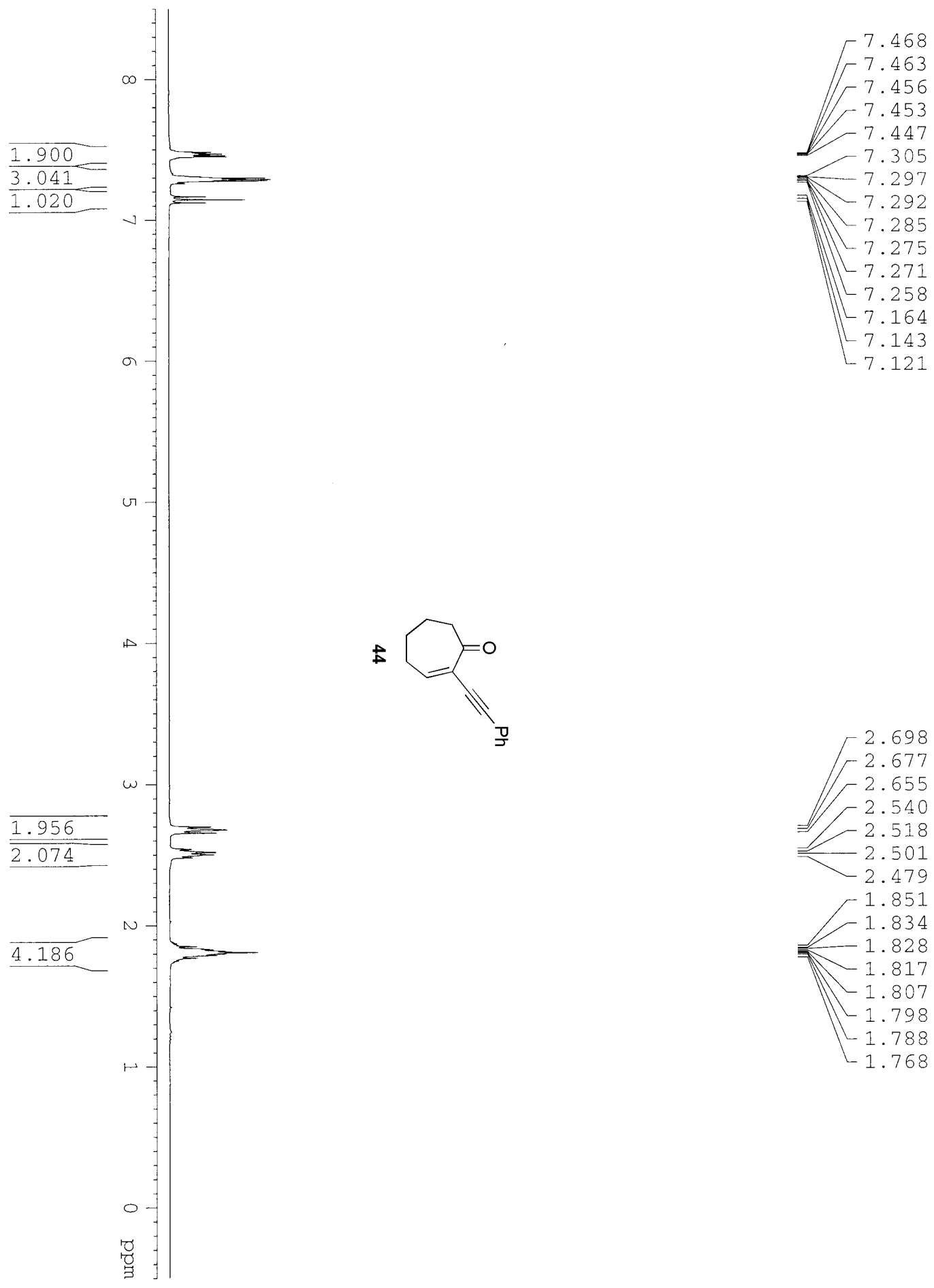


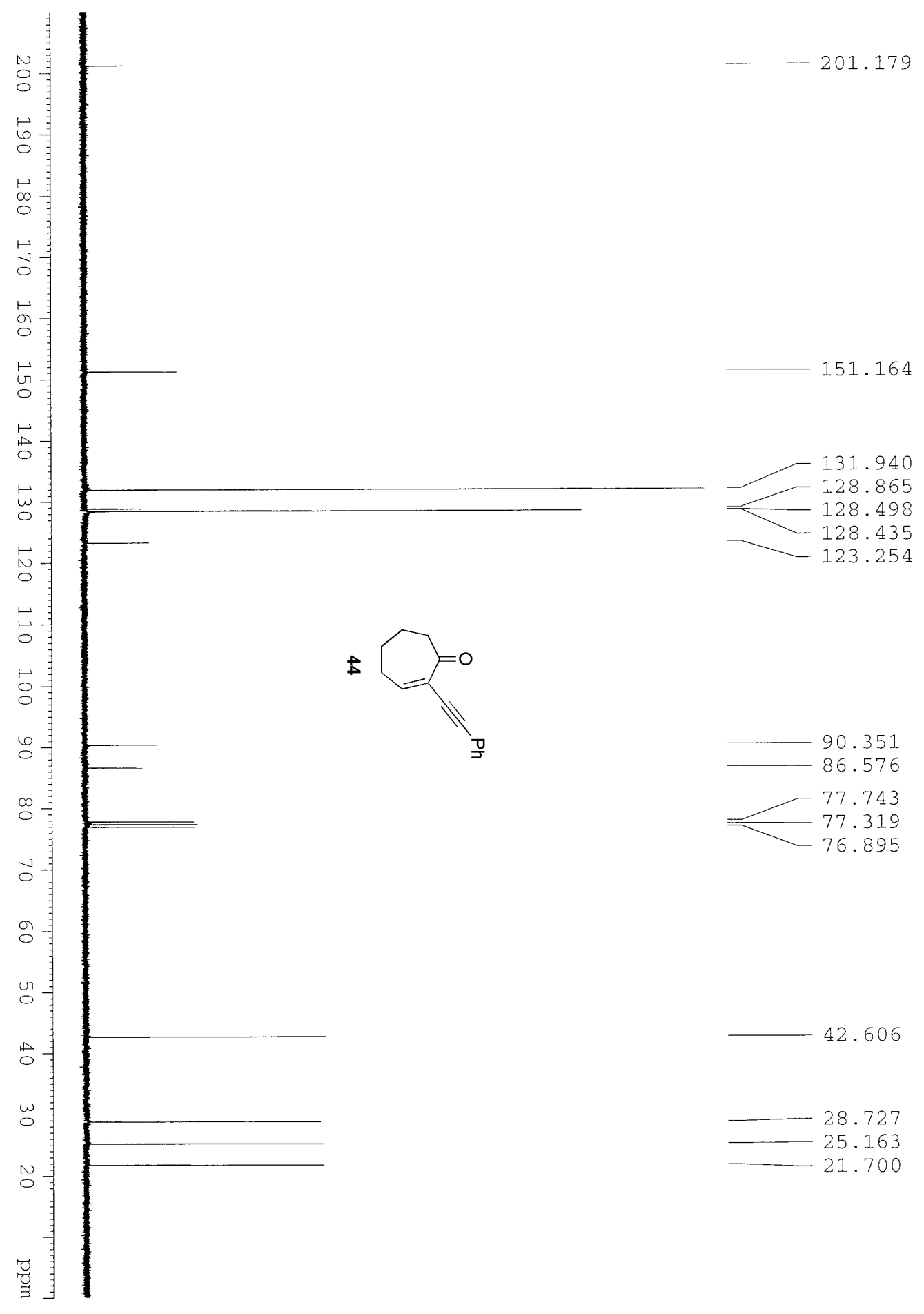



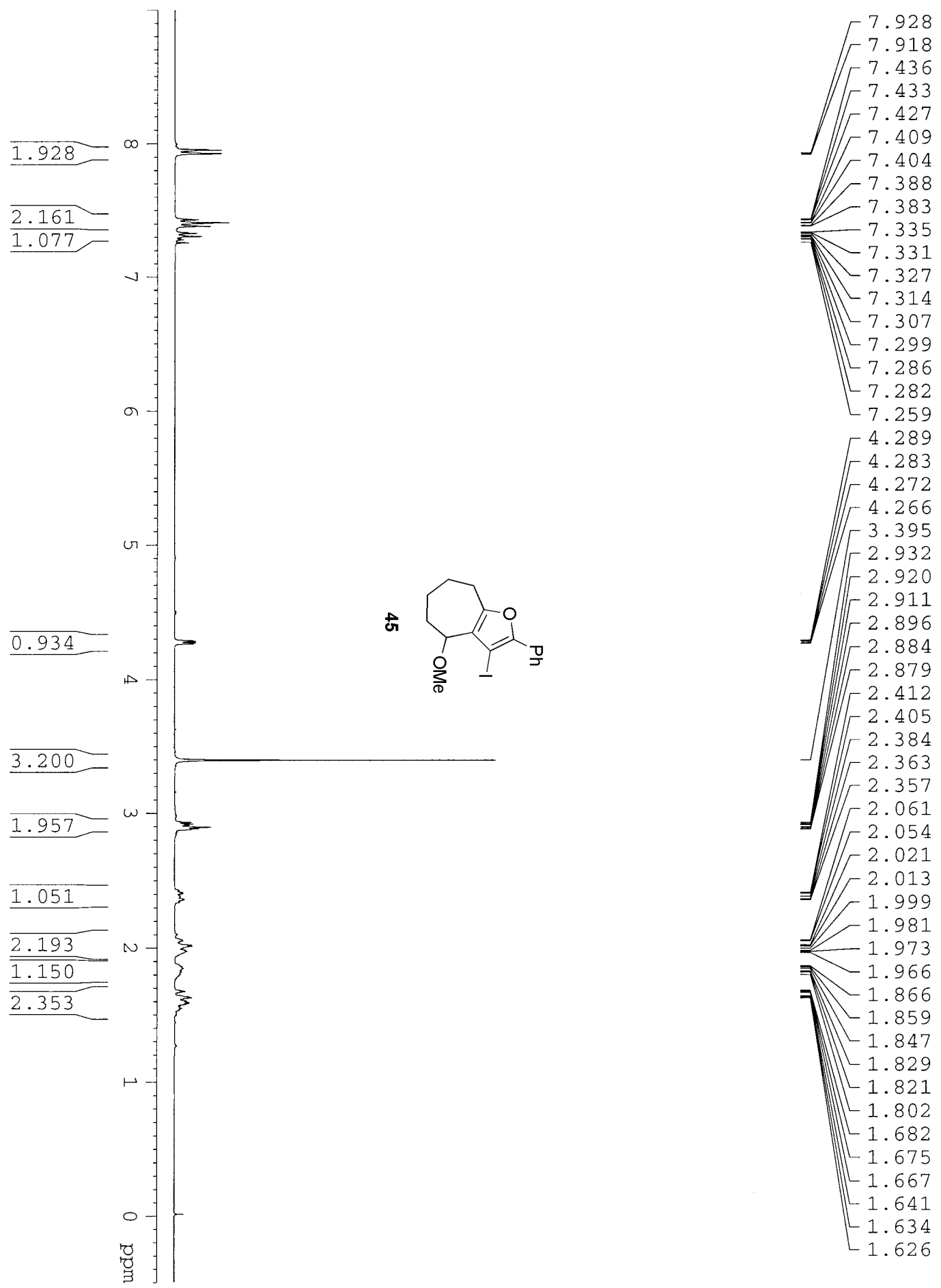

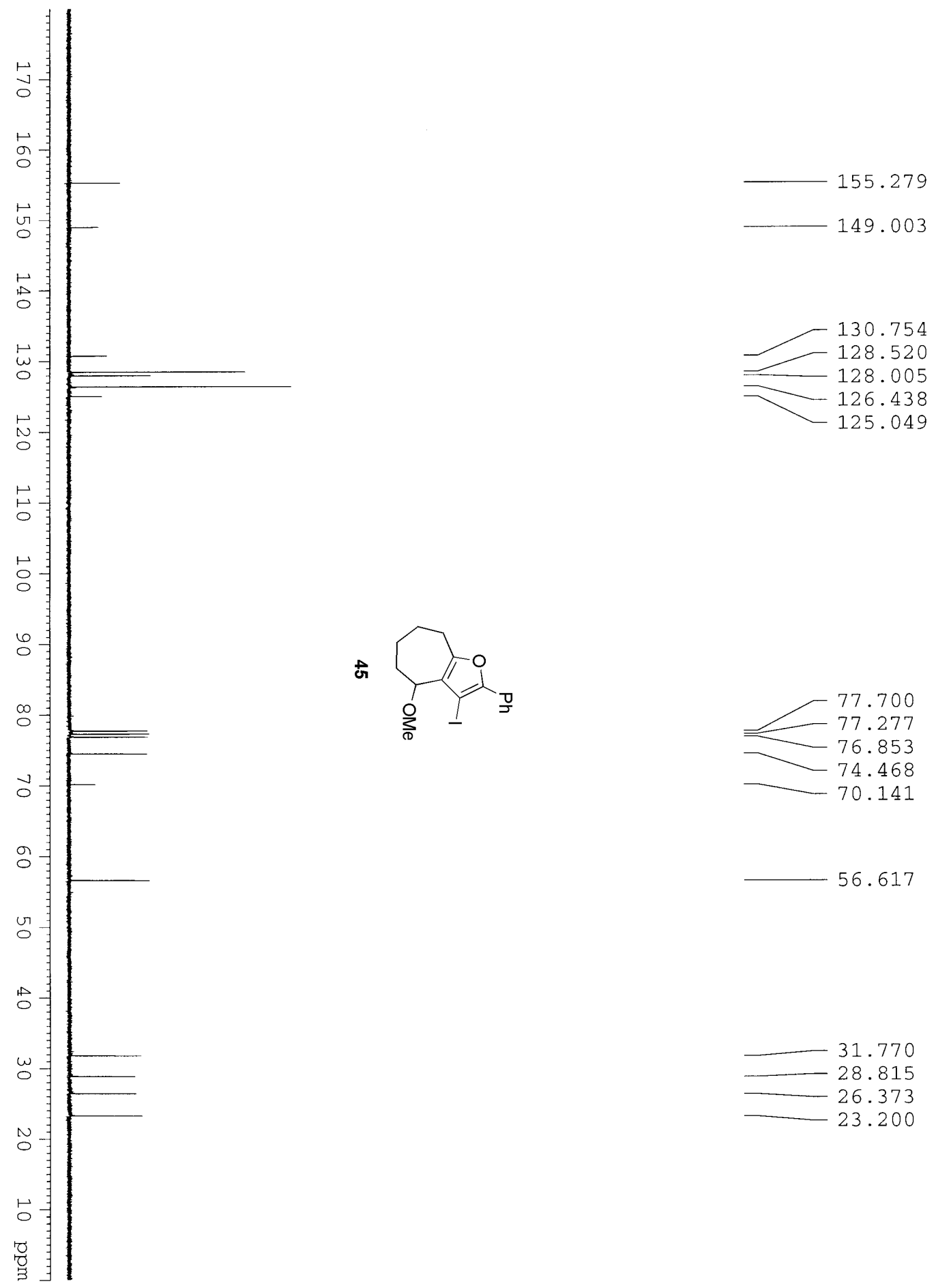

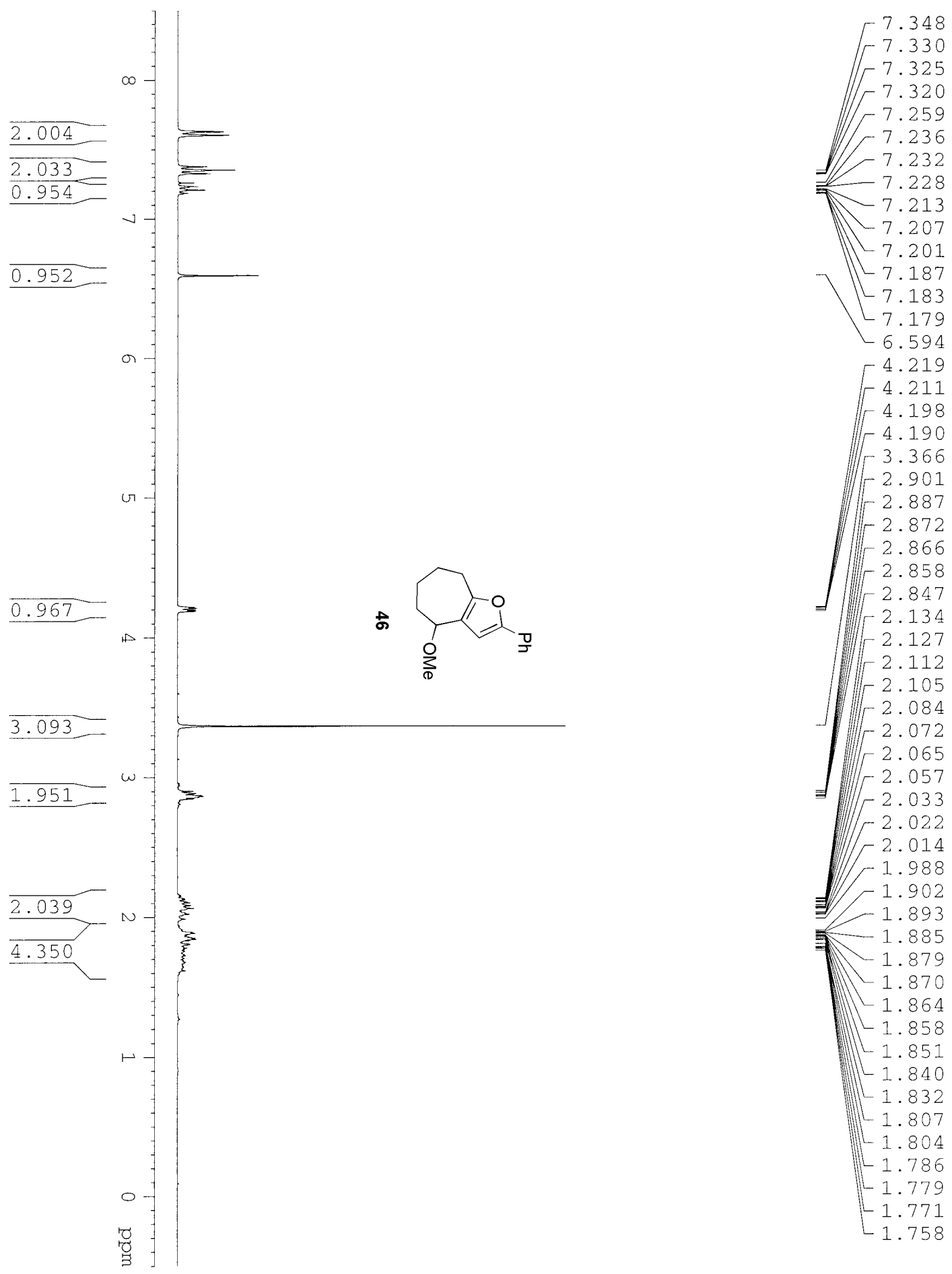

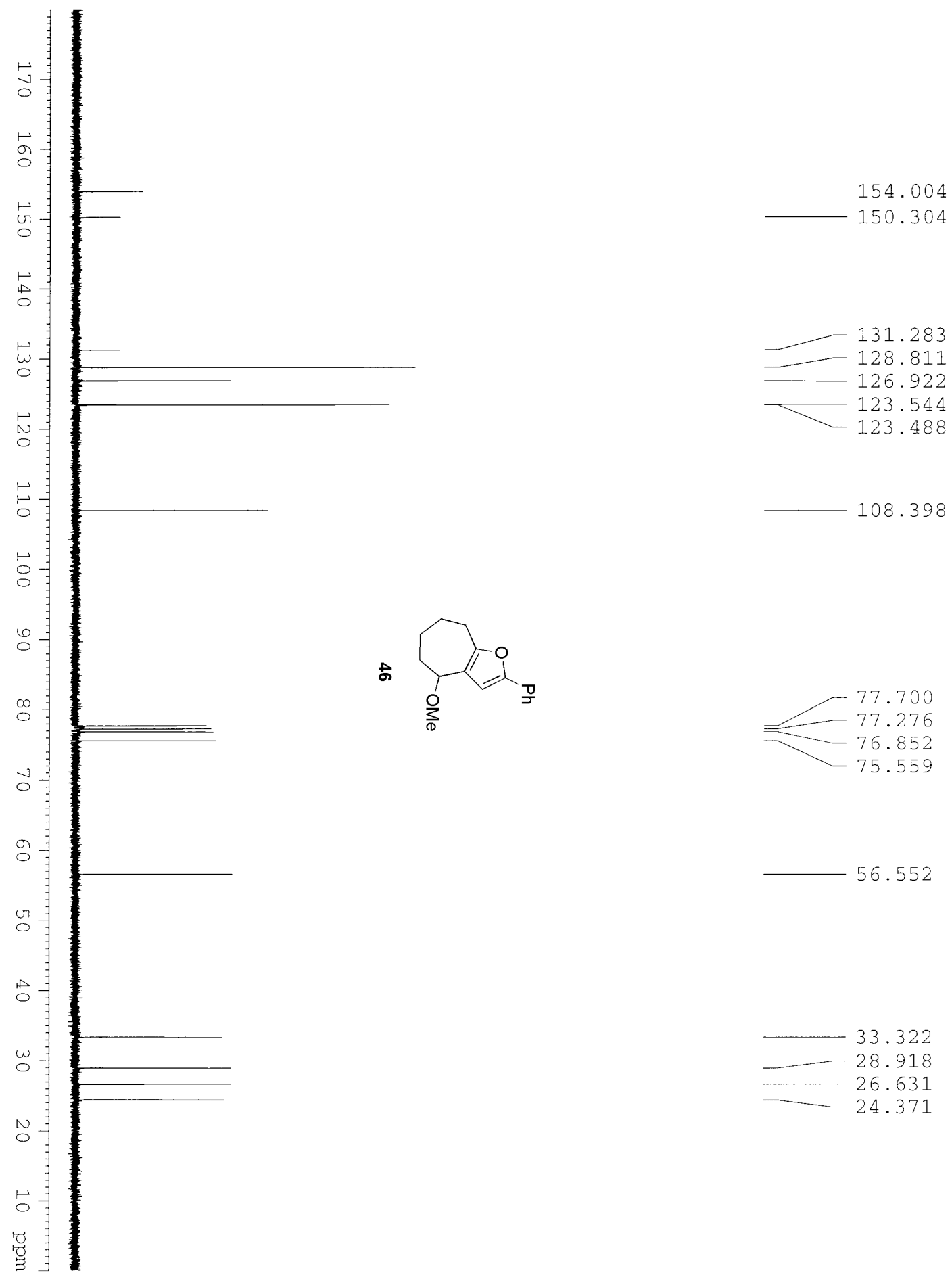

108.398

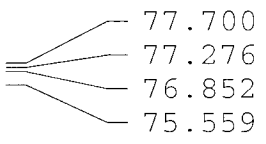

56.552

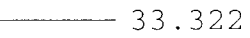

28.918

26.631

24.371 

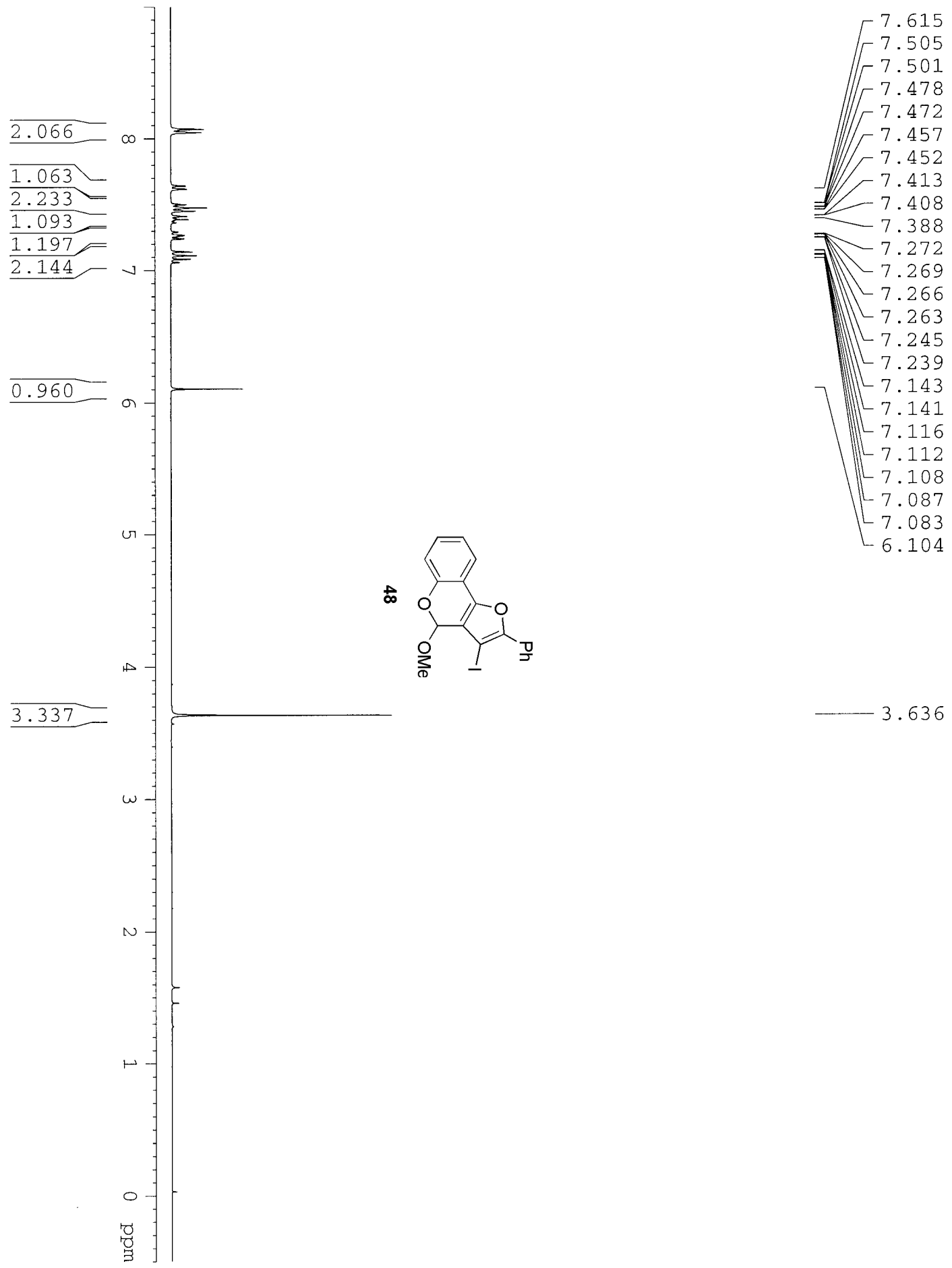

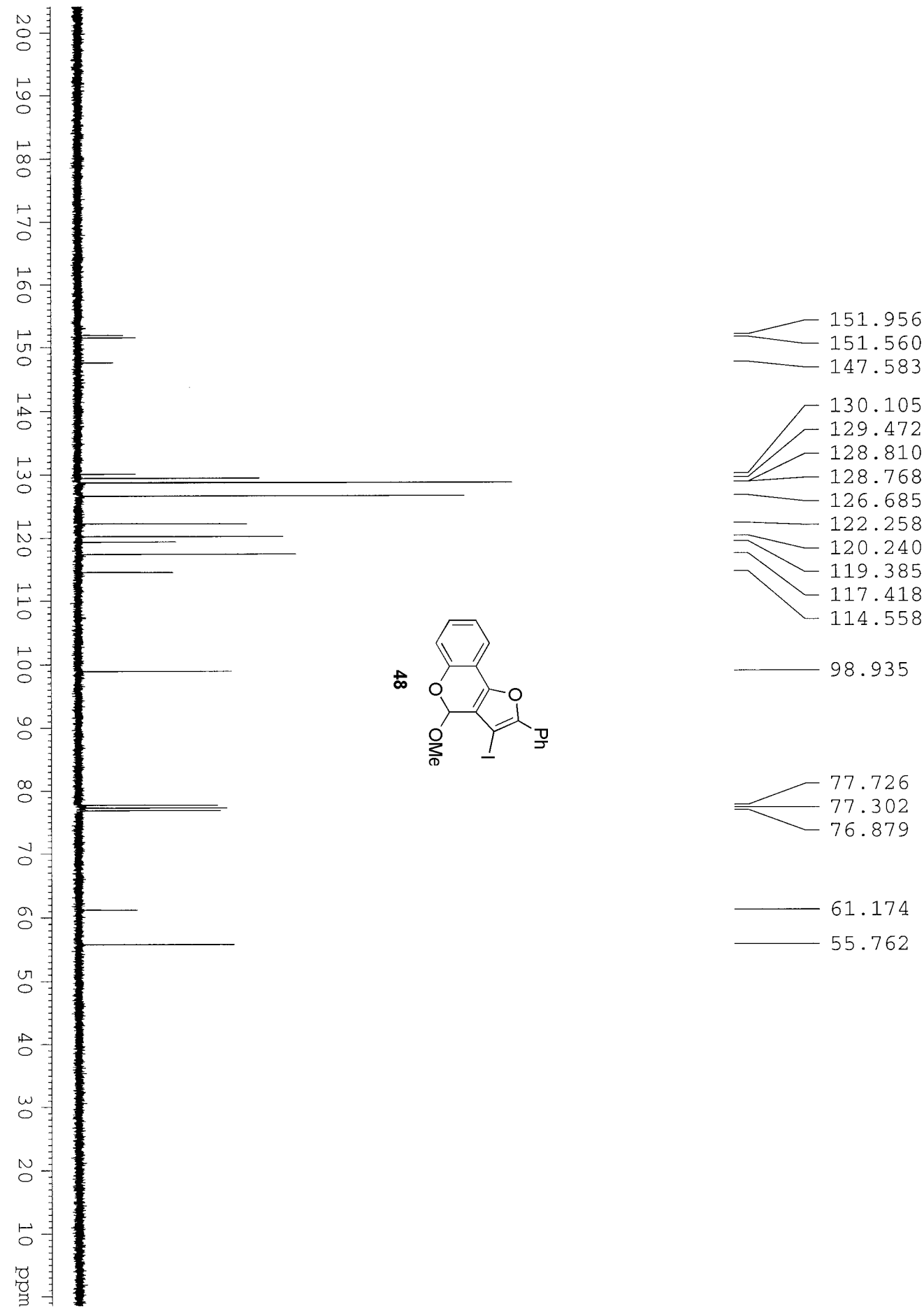

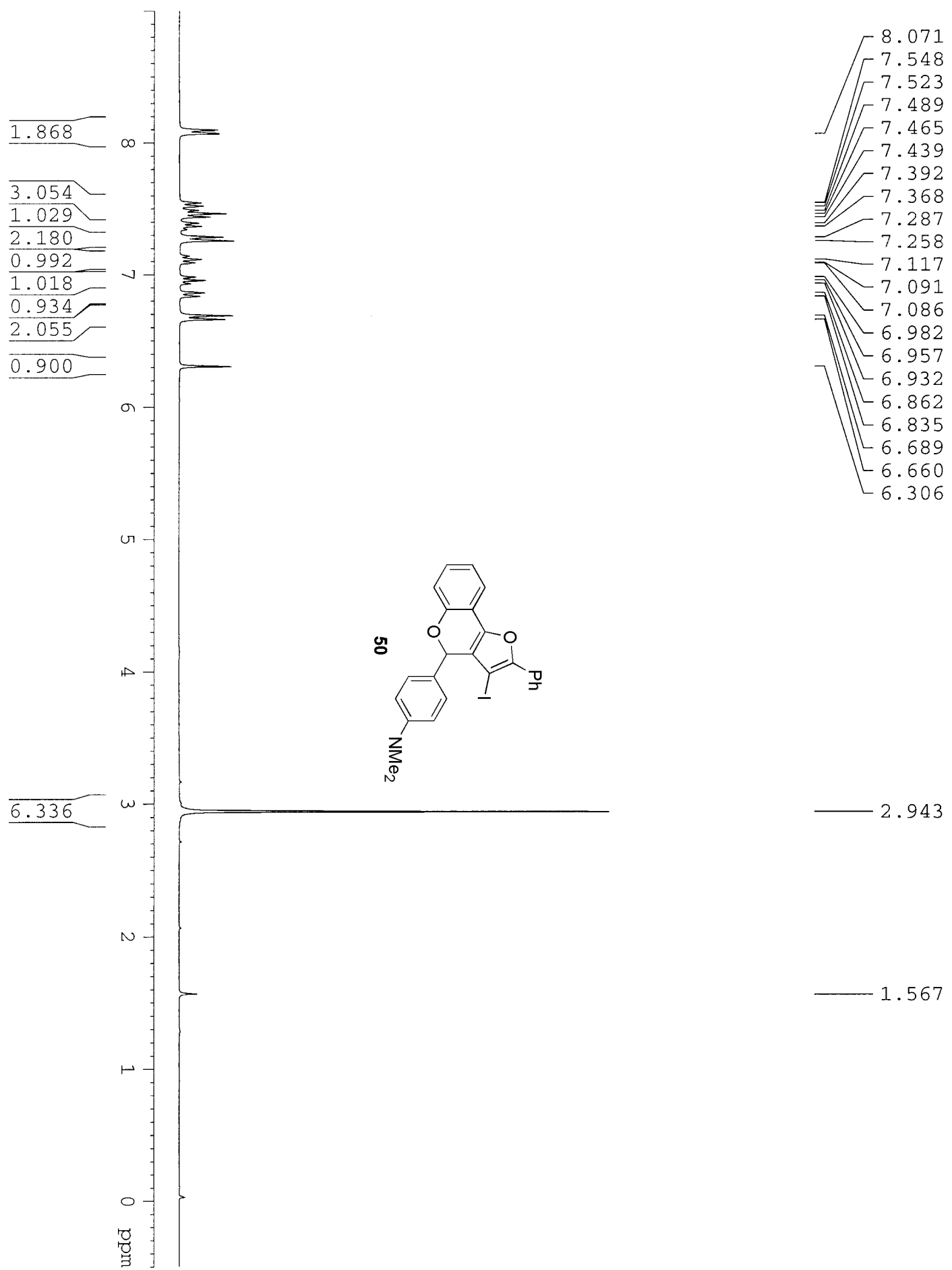

1. 567 

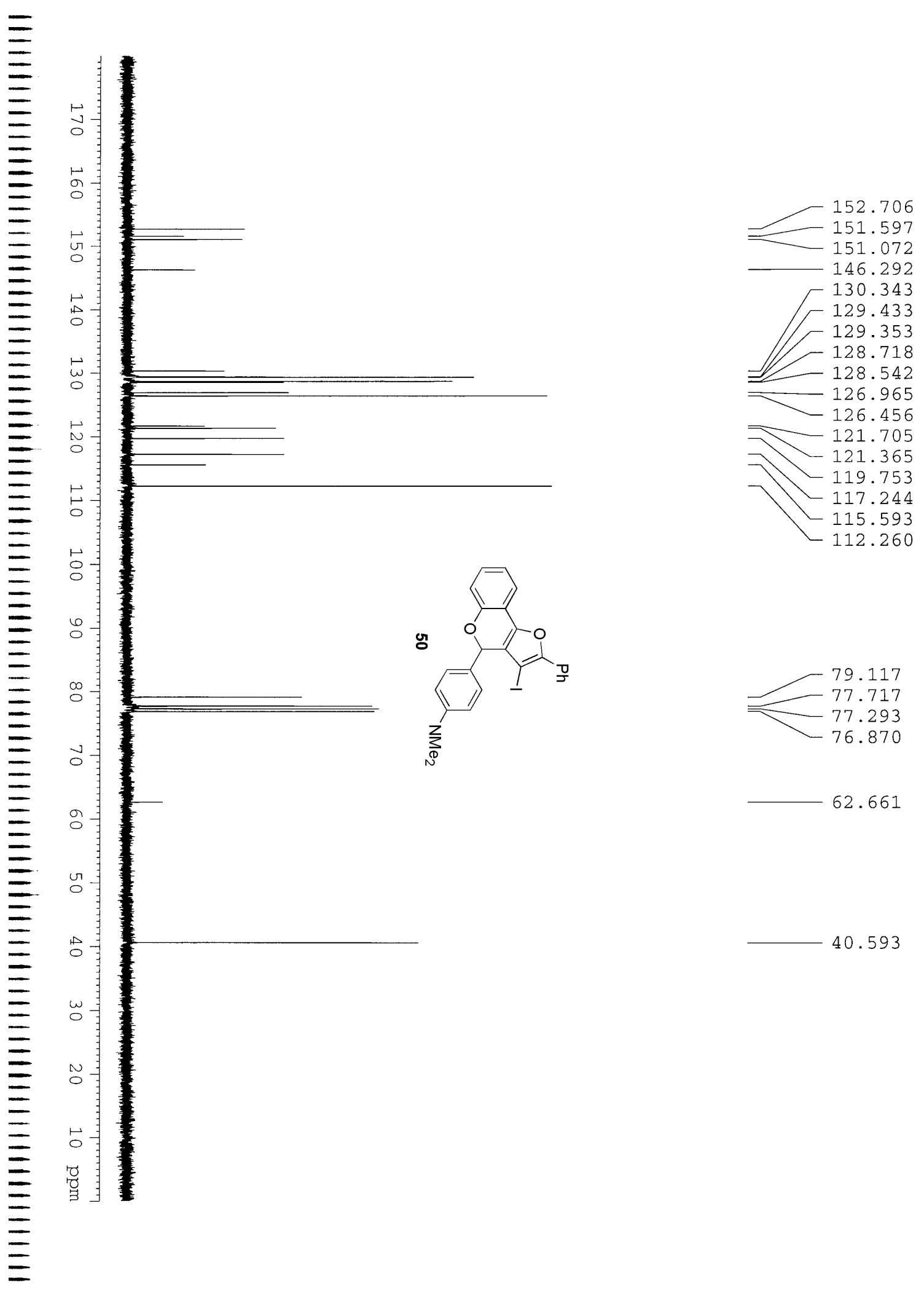

40.593 

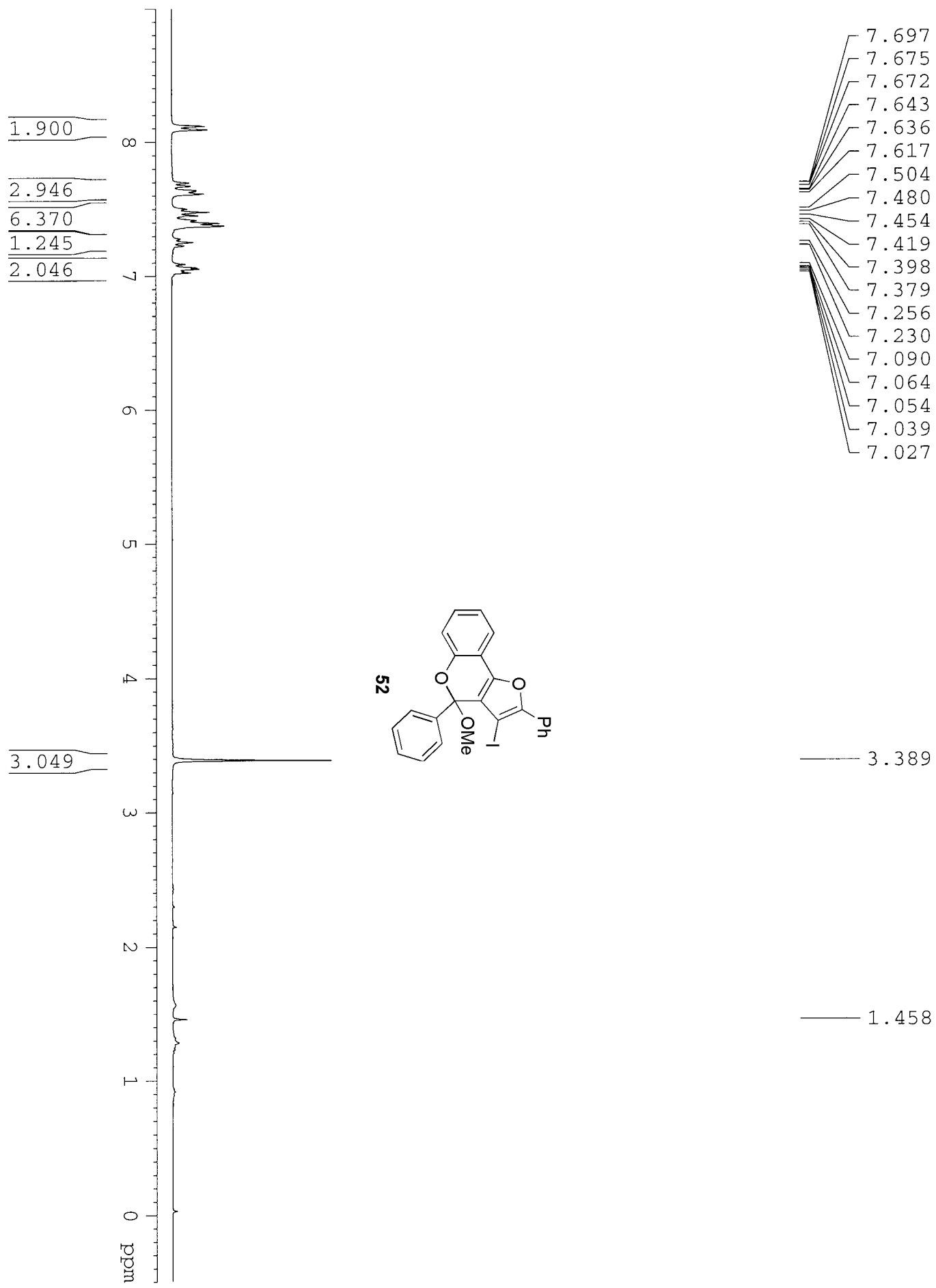

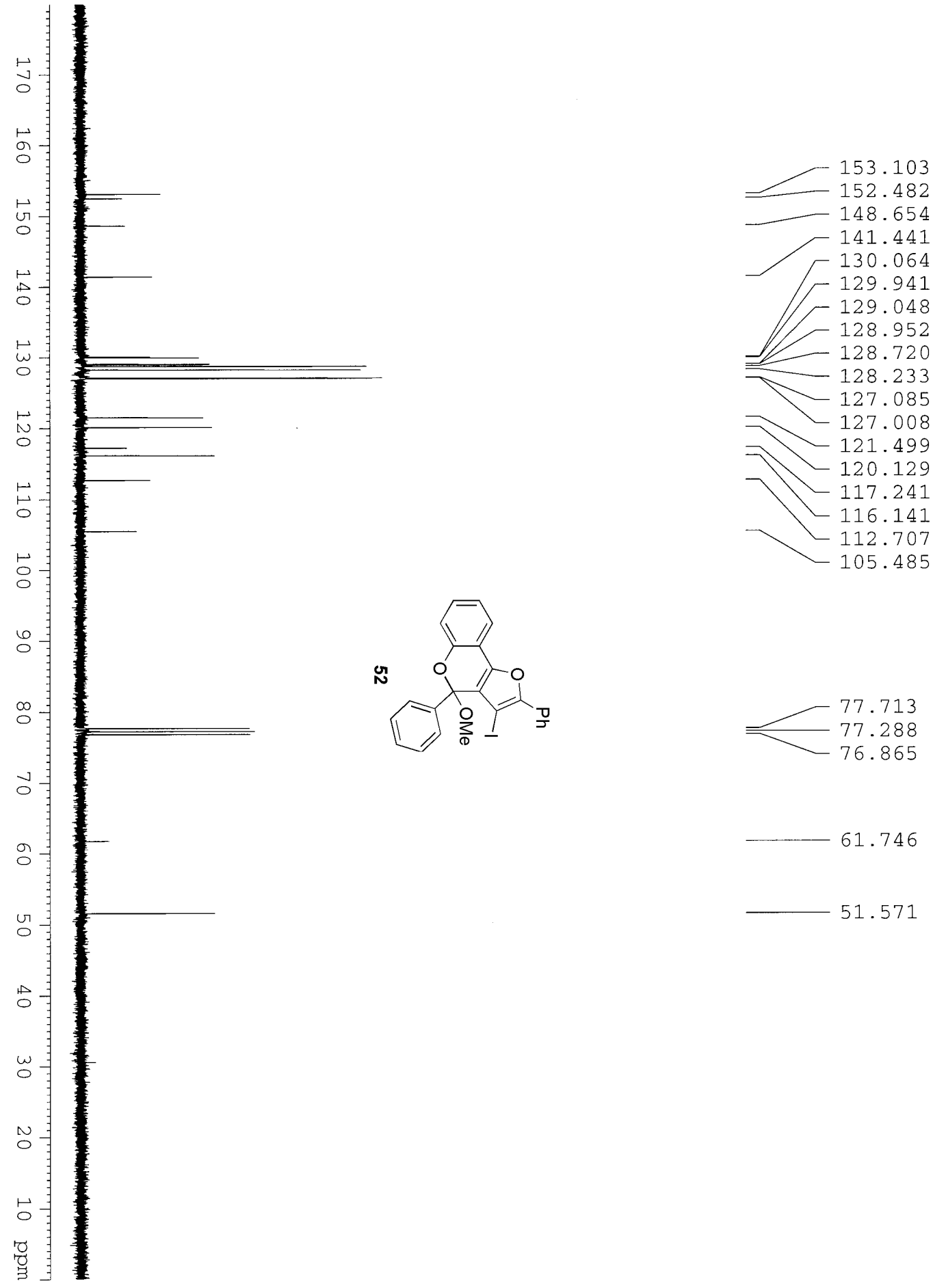

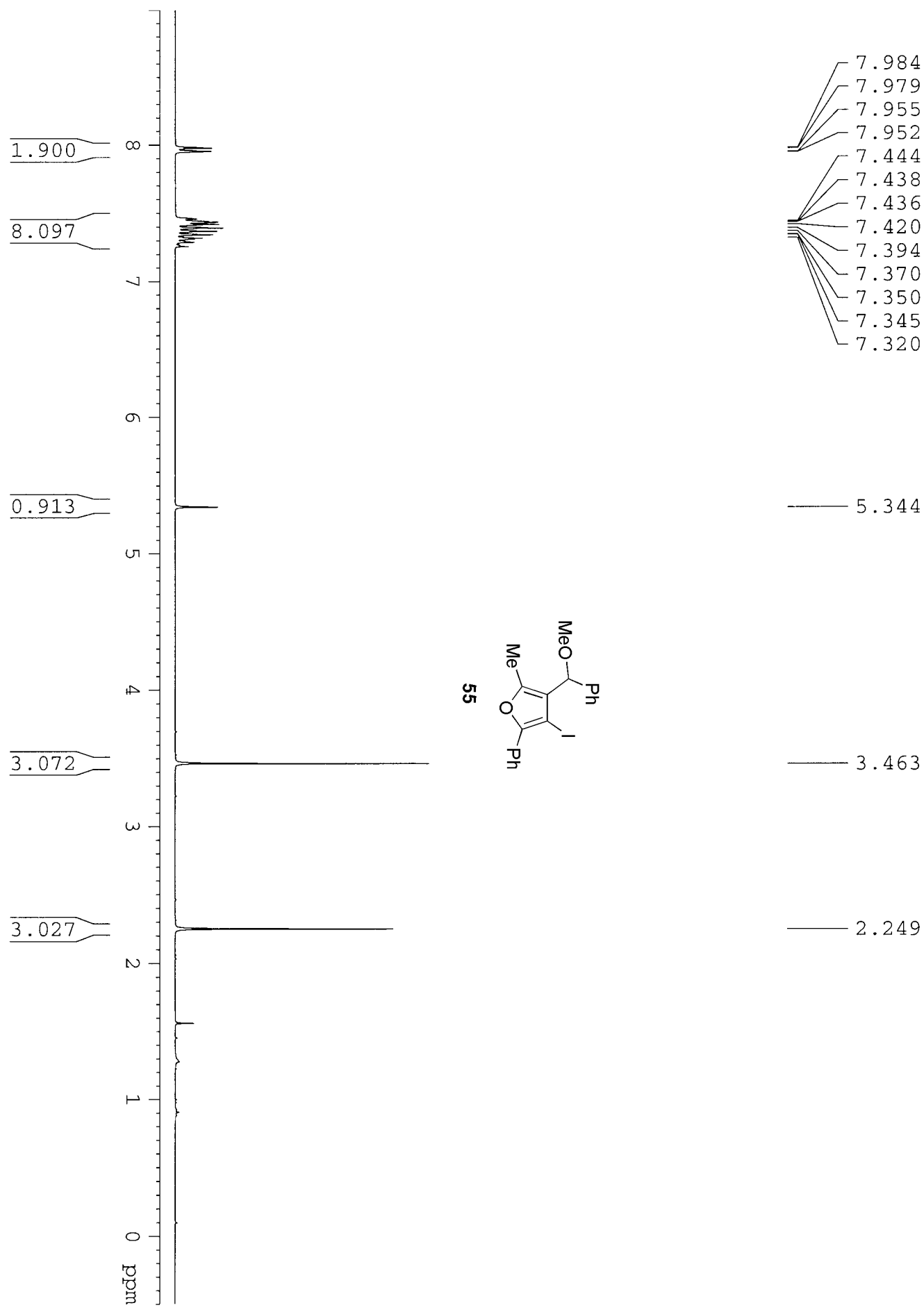

3.463 

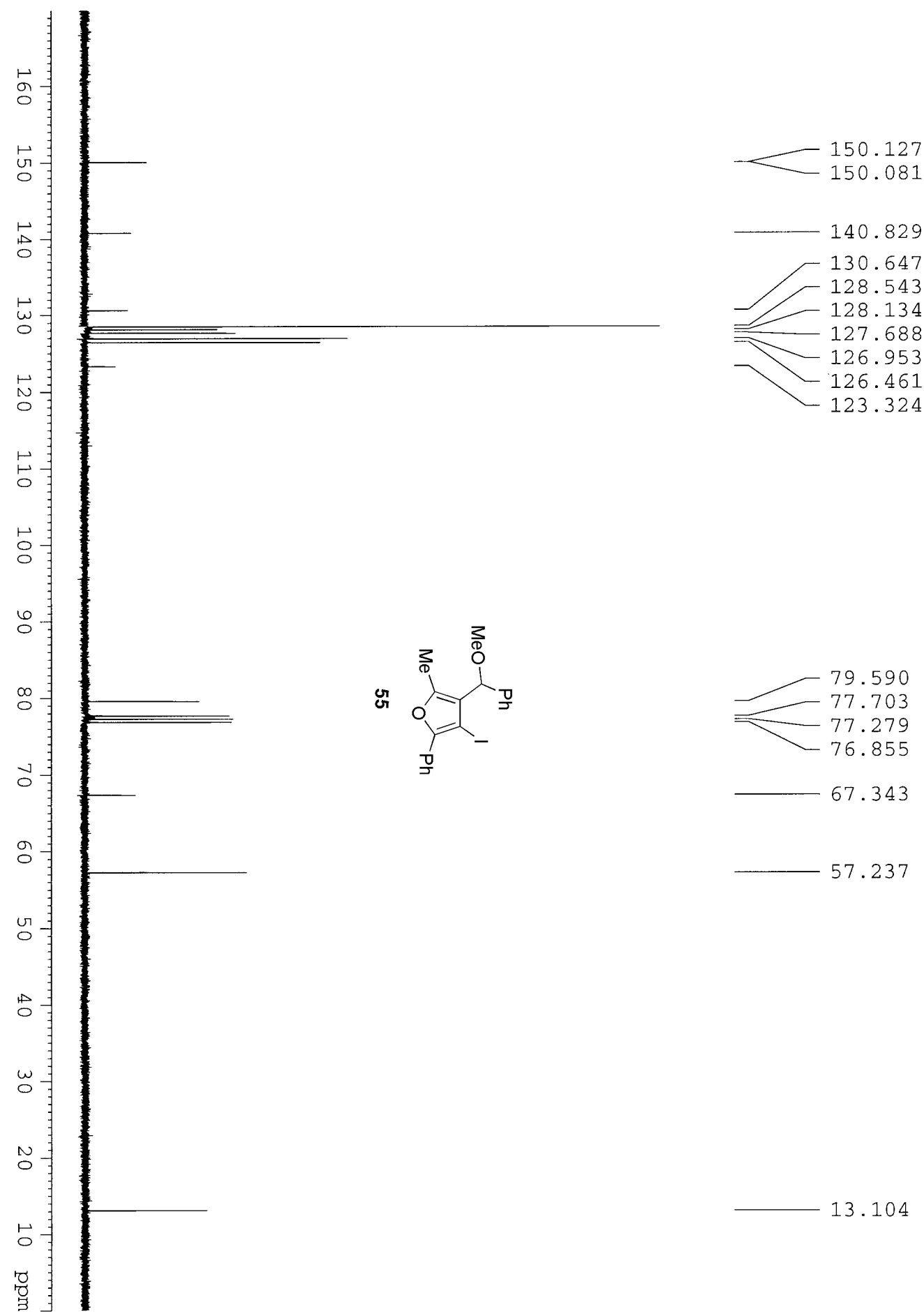

57.237 

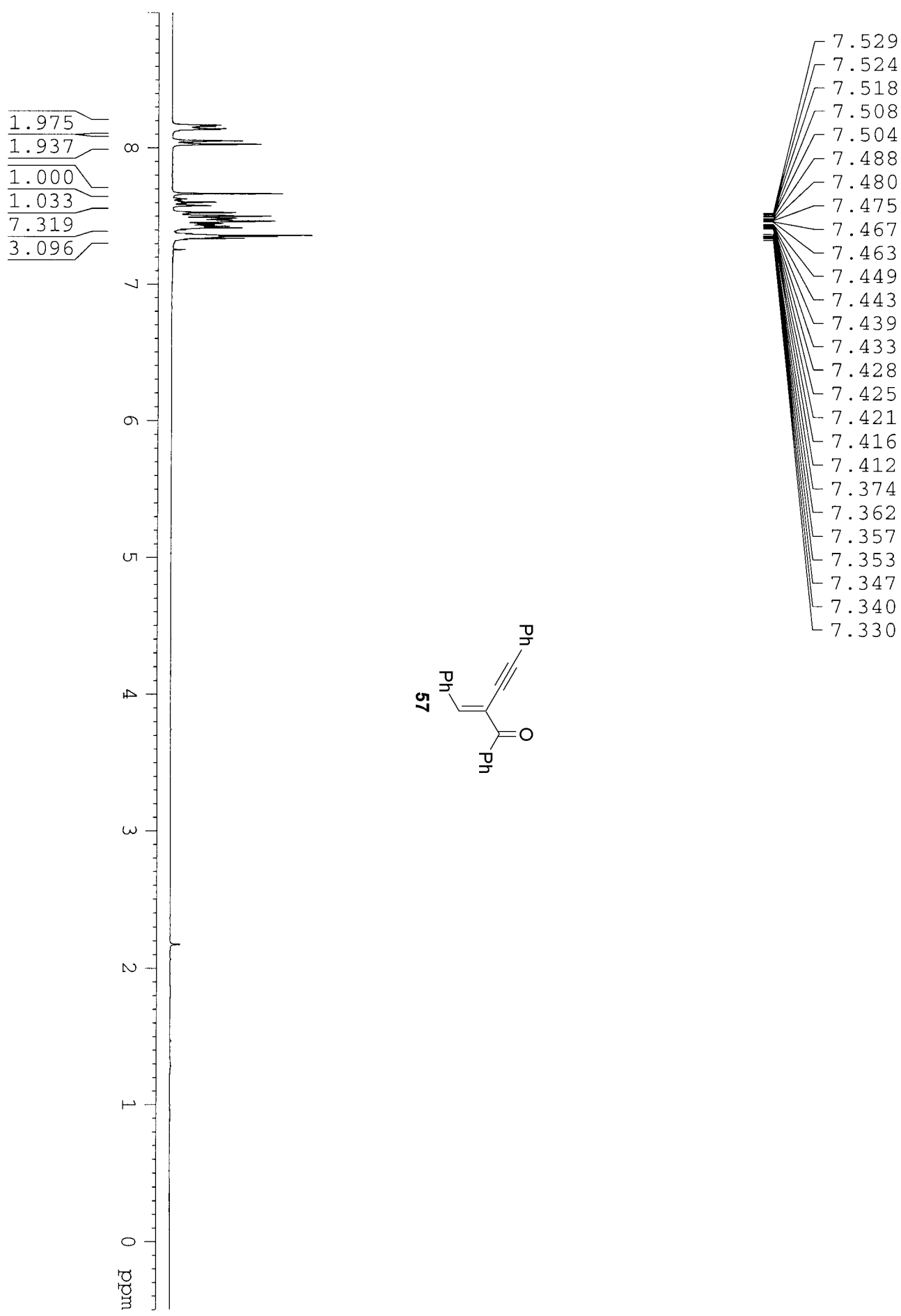

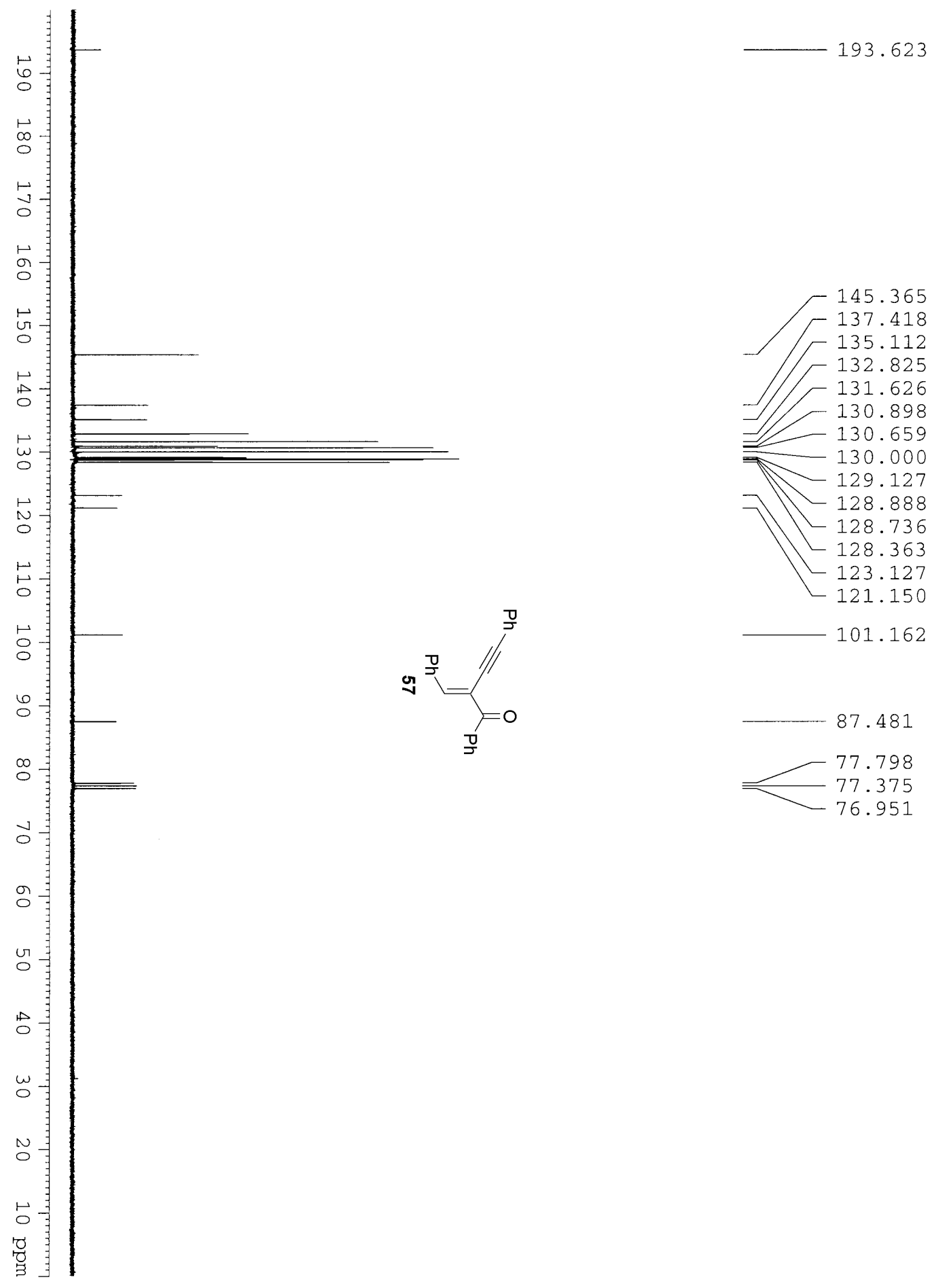

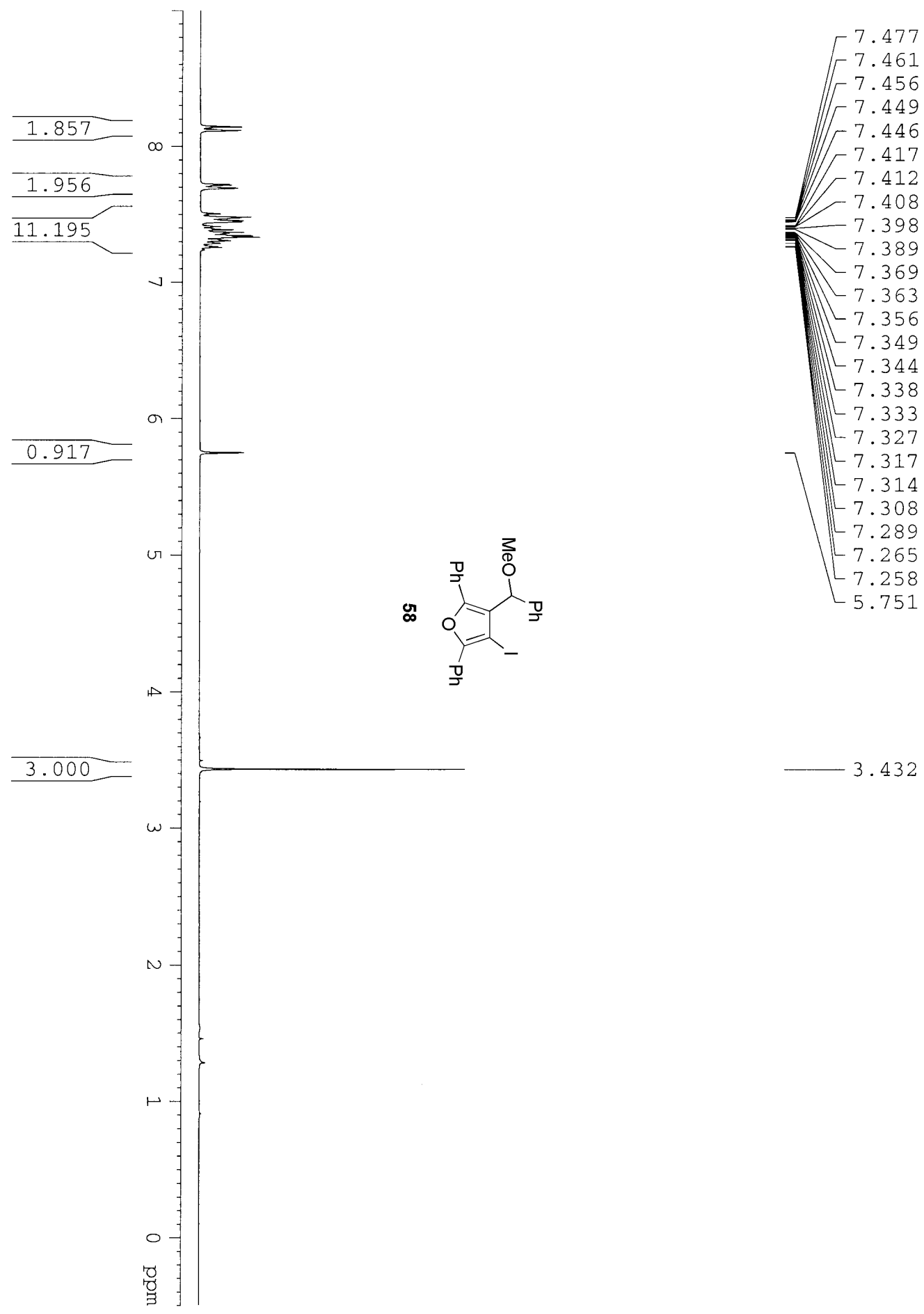

$-3.432$ 

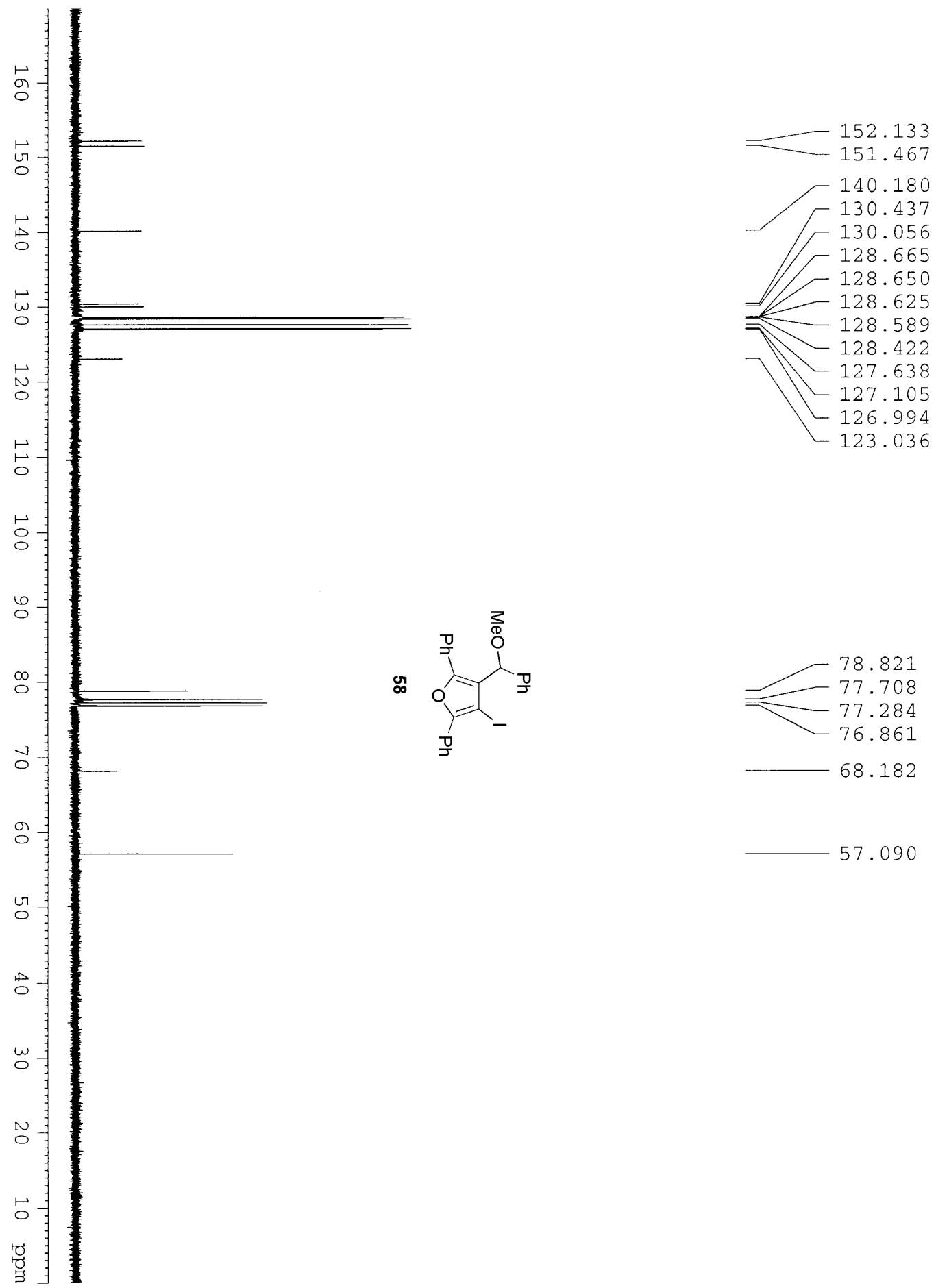

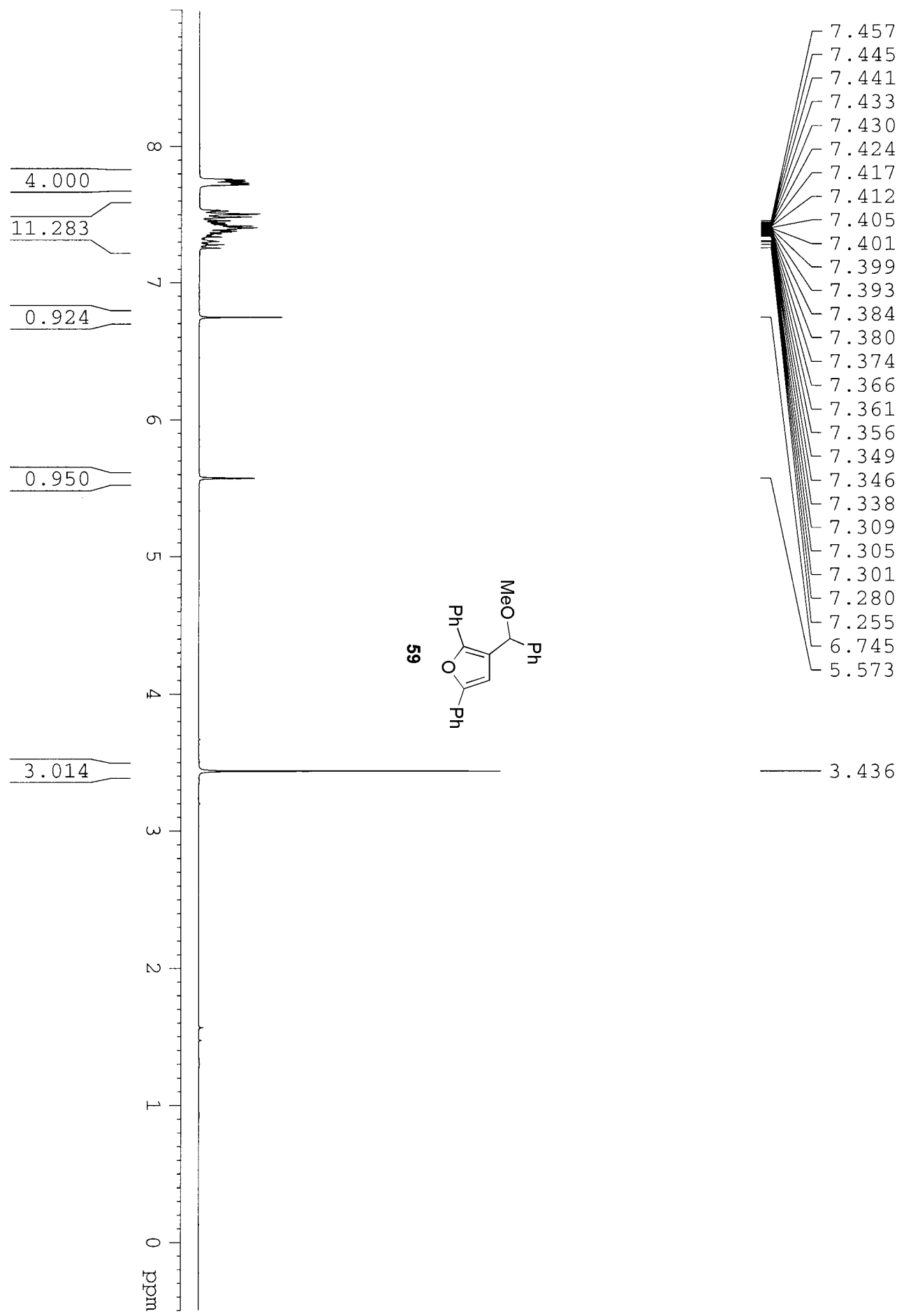

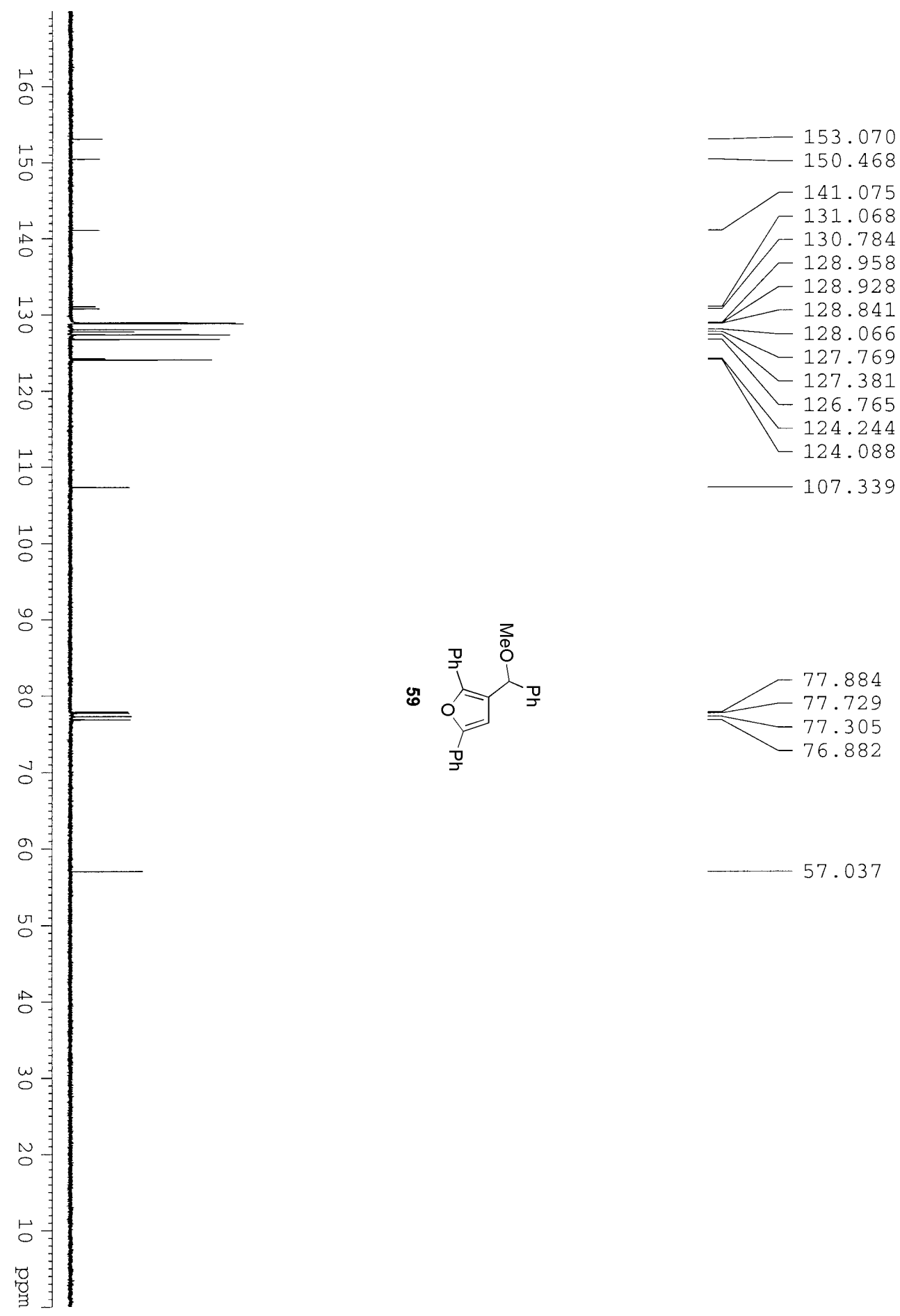

$-57.037$ 

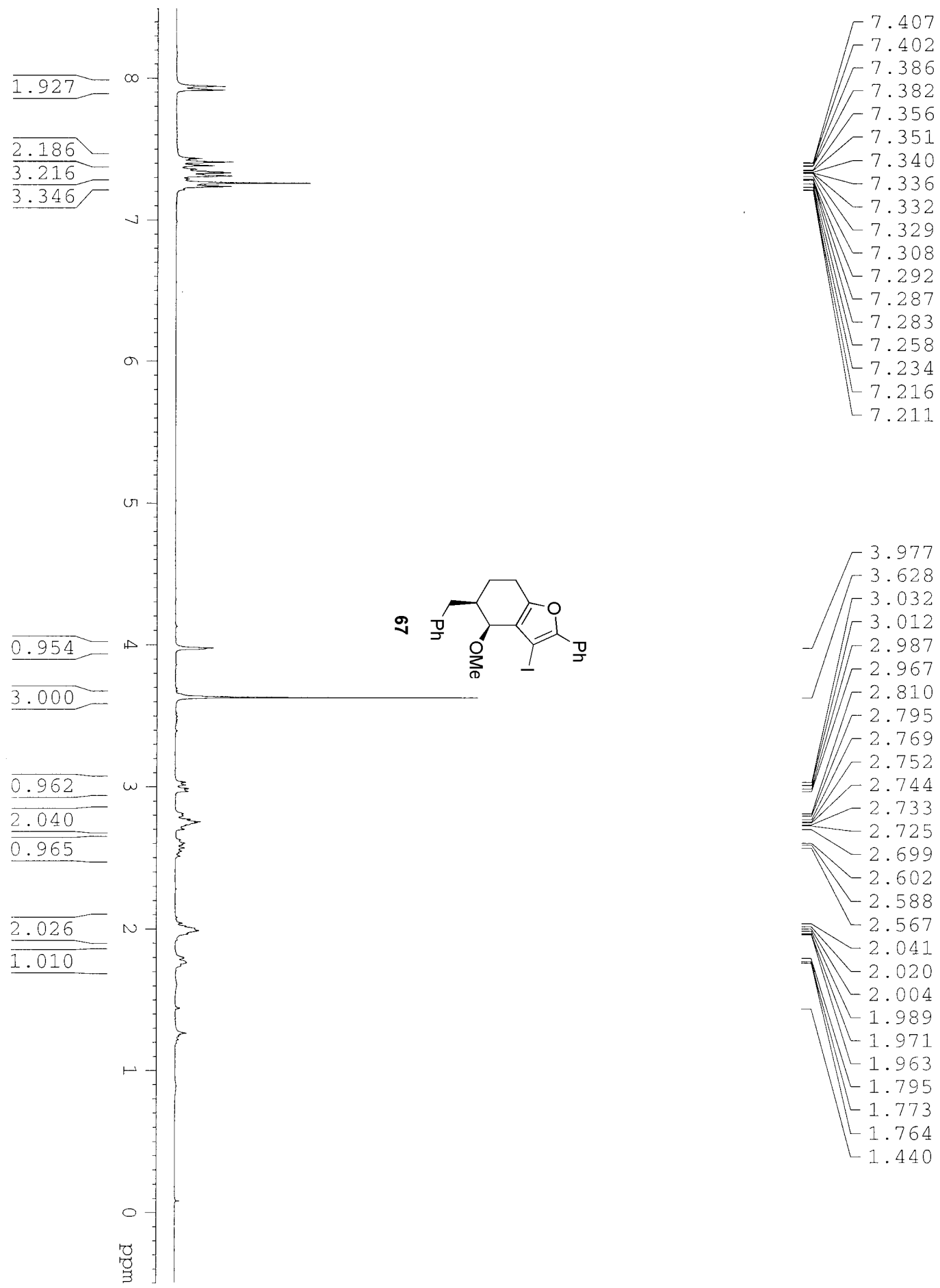

$\Gamma^{2} .810$

$\begin{array}{r}2.795 \\ -2.769\end{array}$

$-2.769$

$\Gamma^{2} .752$

III -2.744

II -2.733
$=2.725$

$\rrbracket 2.699$

$-2.602$

$-2.588$

ㄴ.567

$\mathbb{1}-2.041$

$7-2.020$

$-2.004$

$-1.989$

$-1.971$

$-1.963$

$-1.795$

$-1.773$

1.764

$-1.440$ 

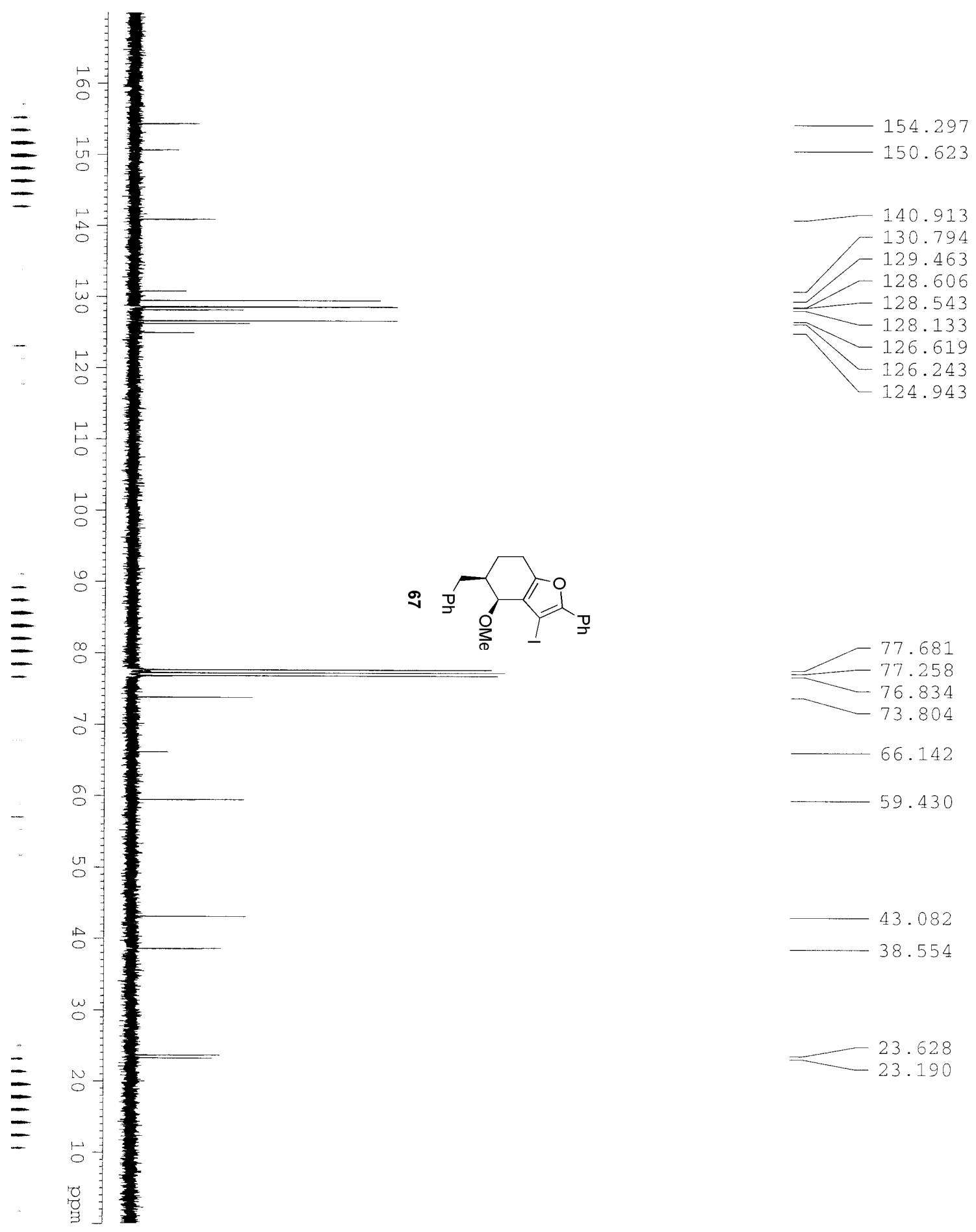

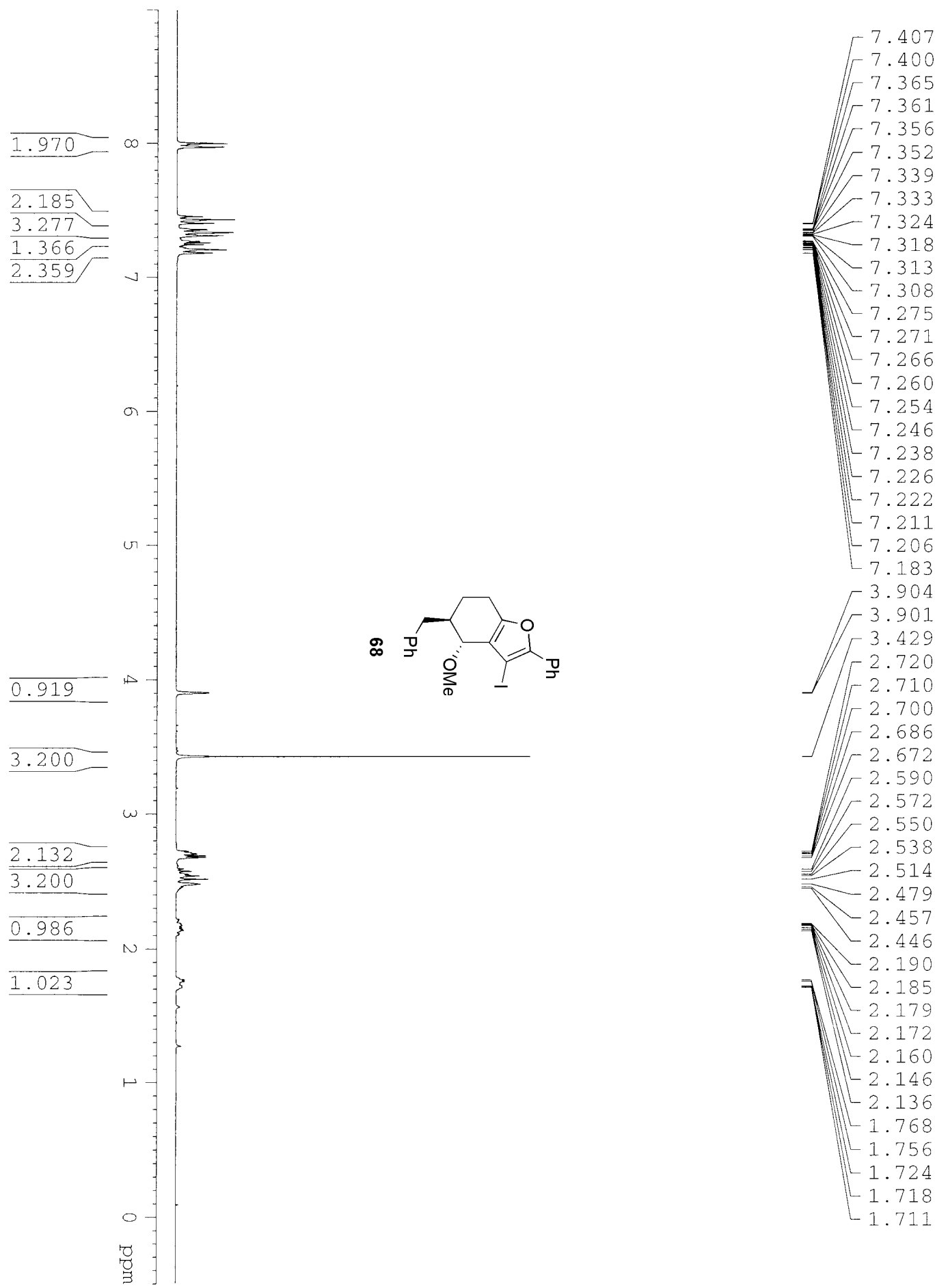

$-3.901$

3.429

$\digamma^{2} .720$

$-2.710$

$-2.700$

$\begin{array}{r}2.686 \\ \hline\end{array}$

- 2.672

$-2.590$

$-2.572$

$-2.550$

$-2.538$

$-2.514$

$-2.457$

$\mathbb{1}-2.446$

$-2.190$

7 $\mid-2.185$

$\lfloor 2.179$

II 2.172

42.160

$-2.146$

$-2.136$

L 1.768

41.756

1.724

L 1.718

$-1.711$ 

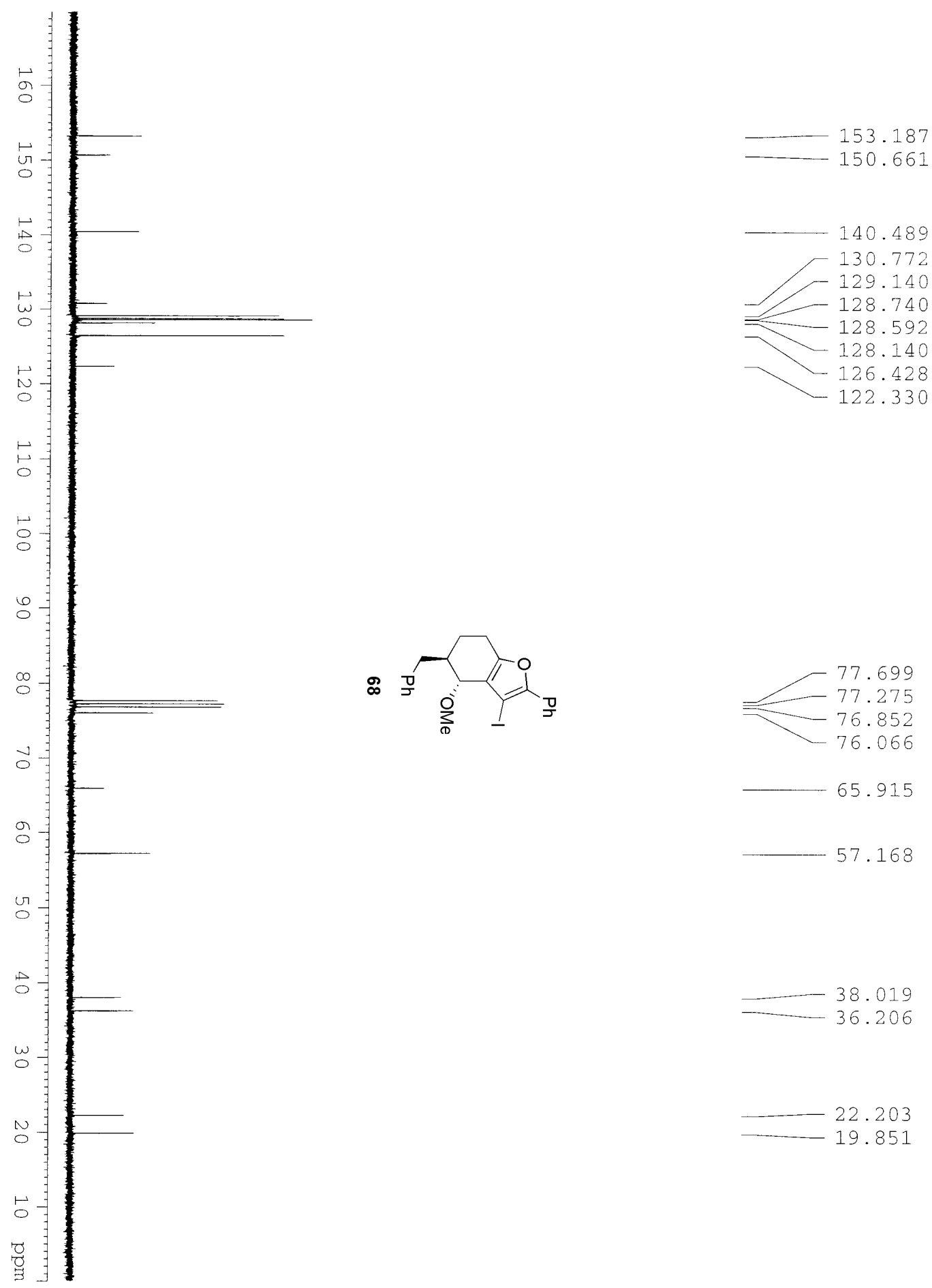

22.203

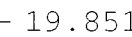



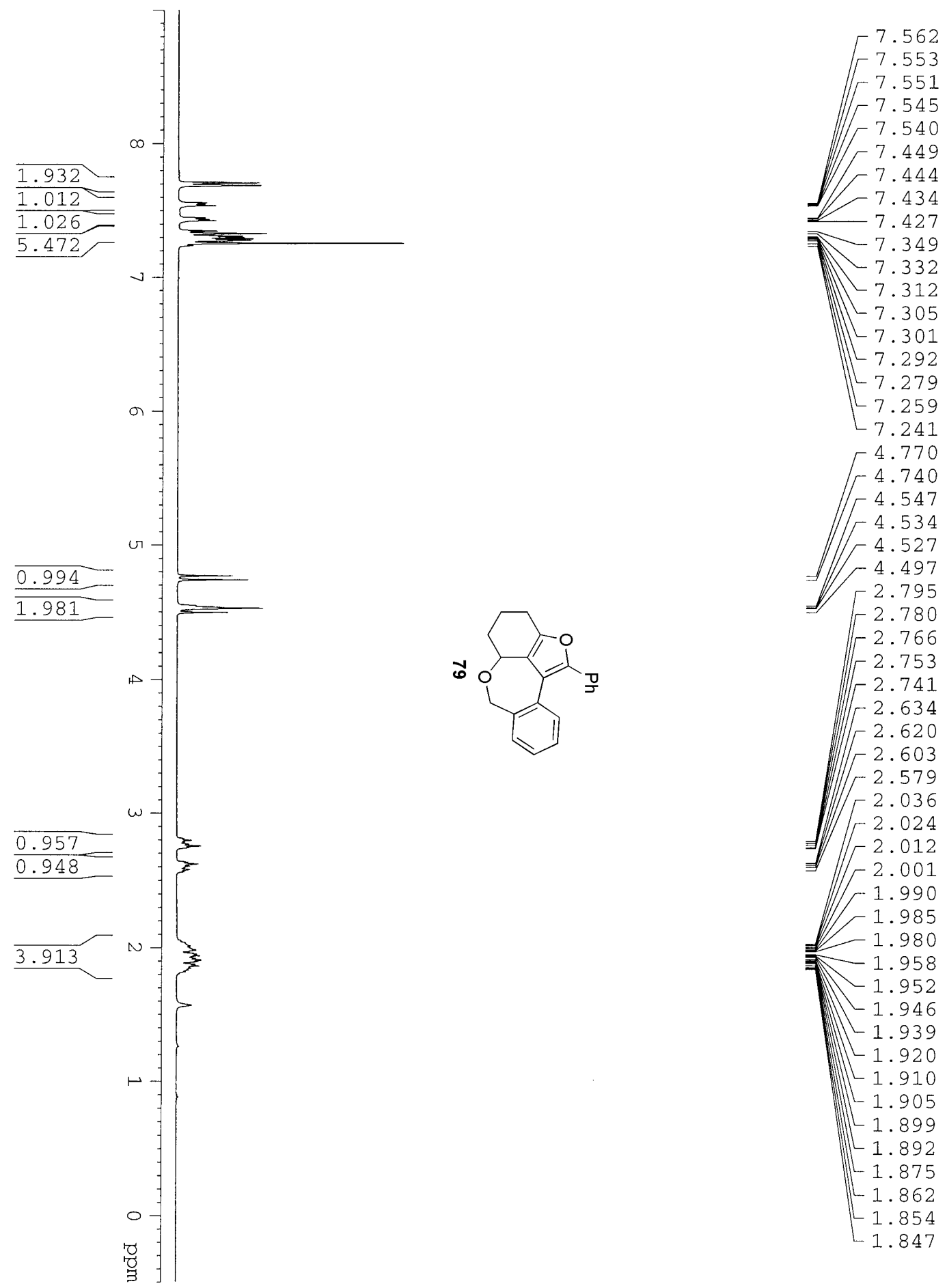

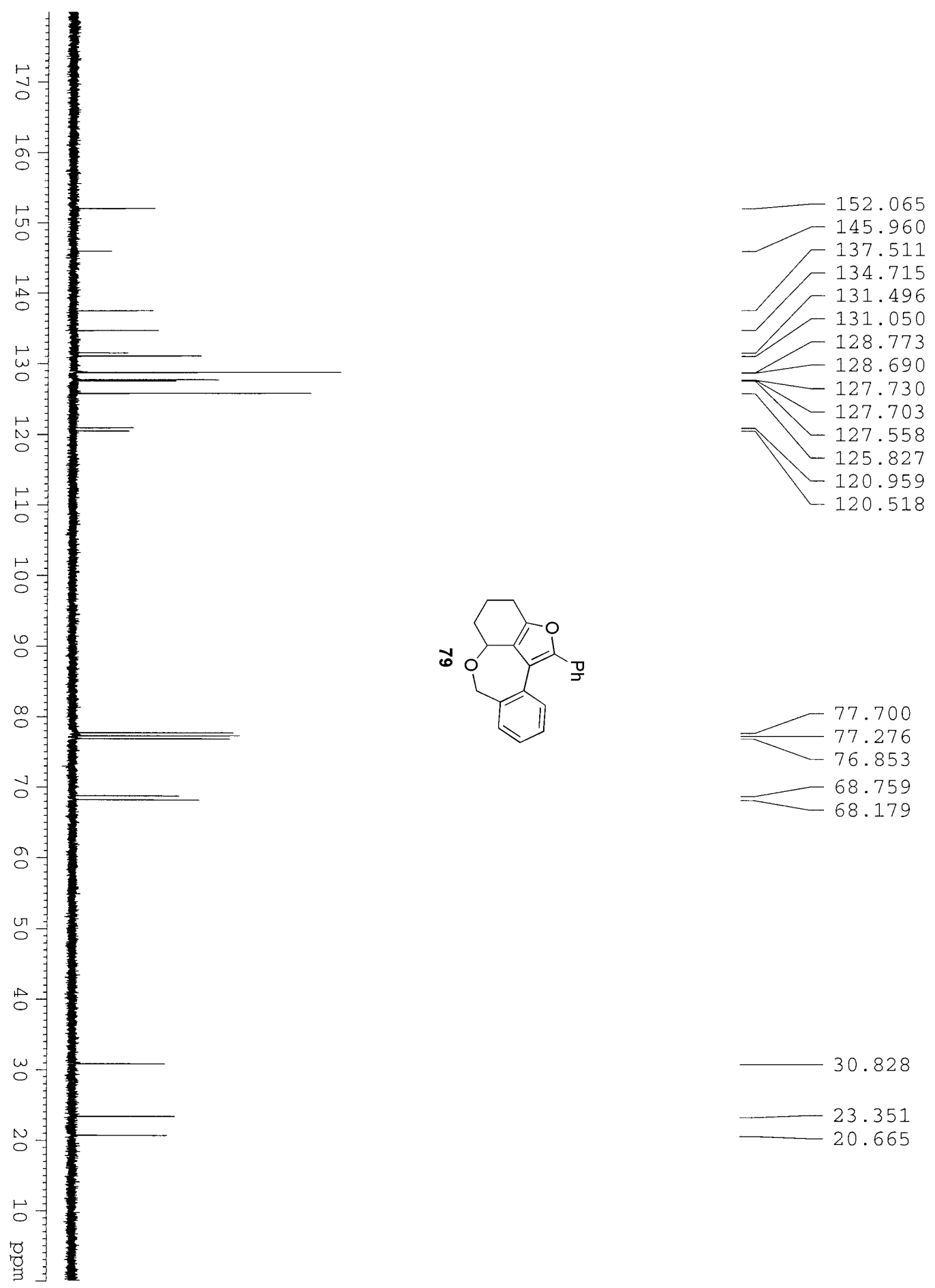

30.828

23.351

20.665 

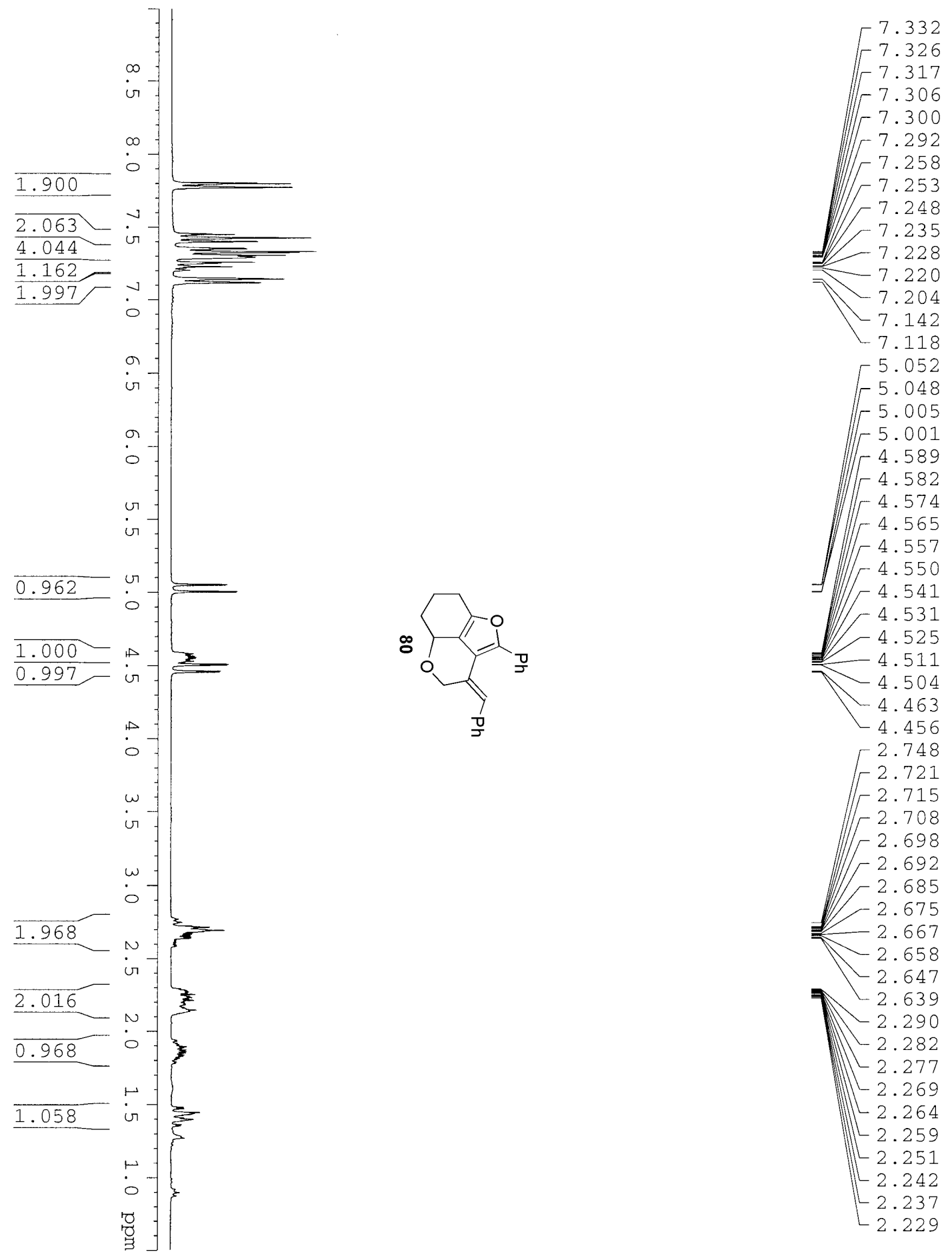

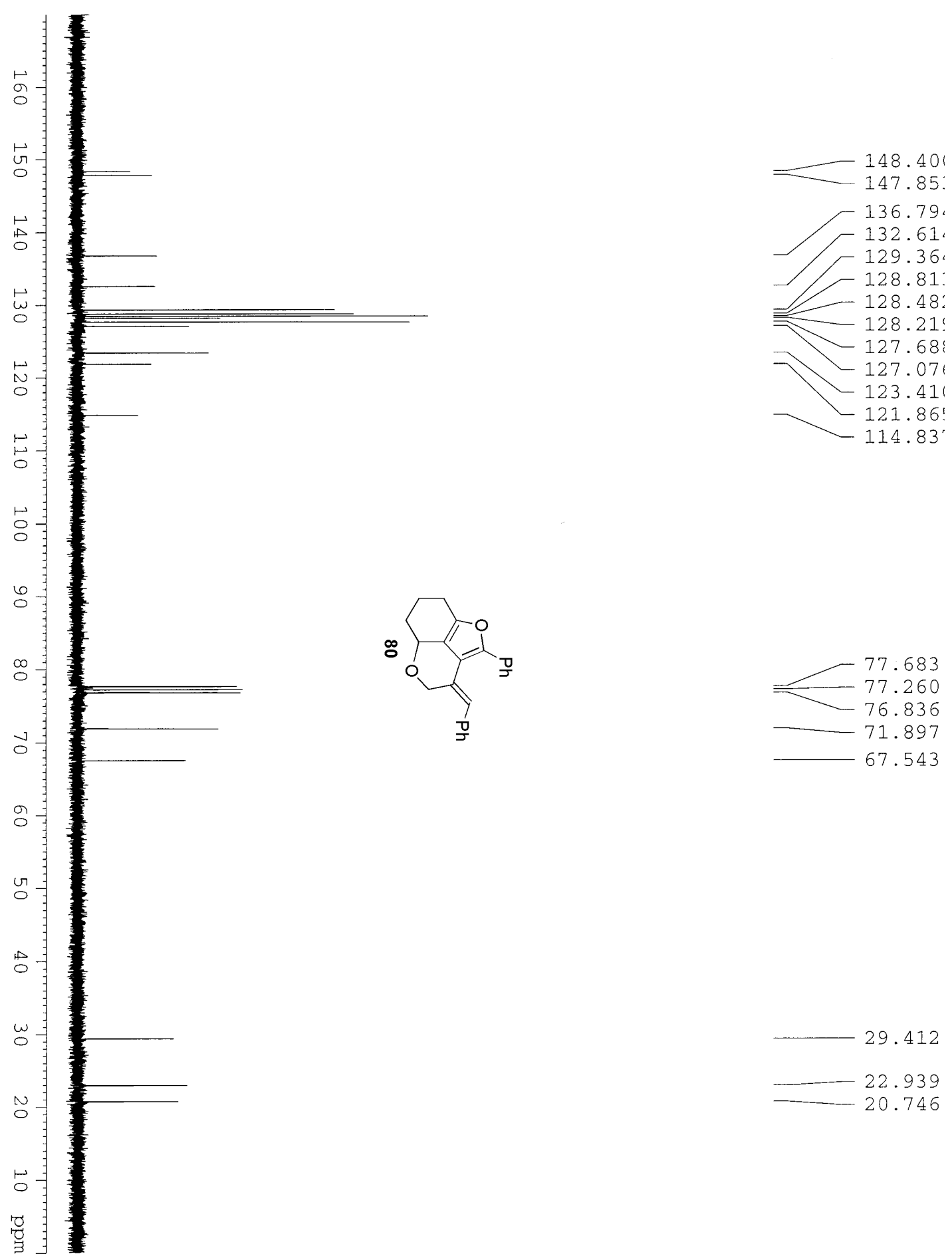

29.412

22.939

20.7 

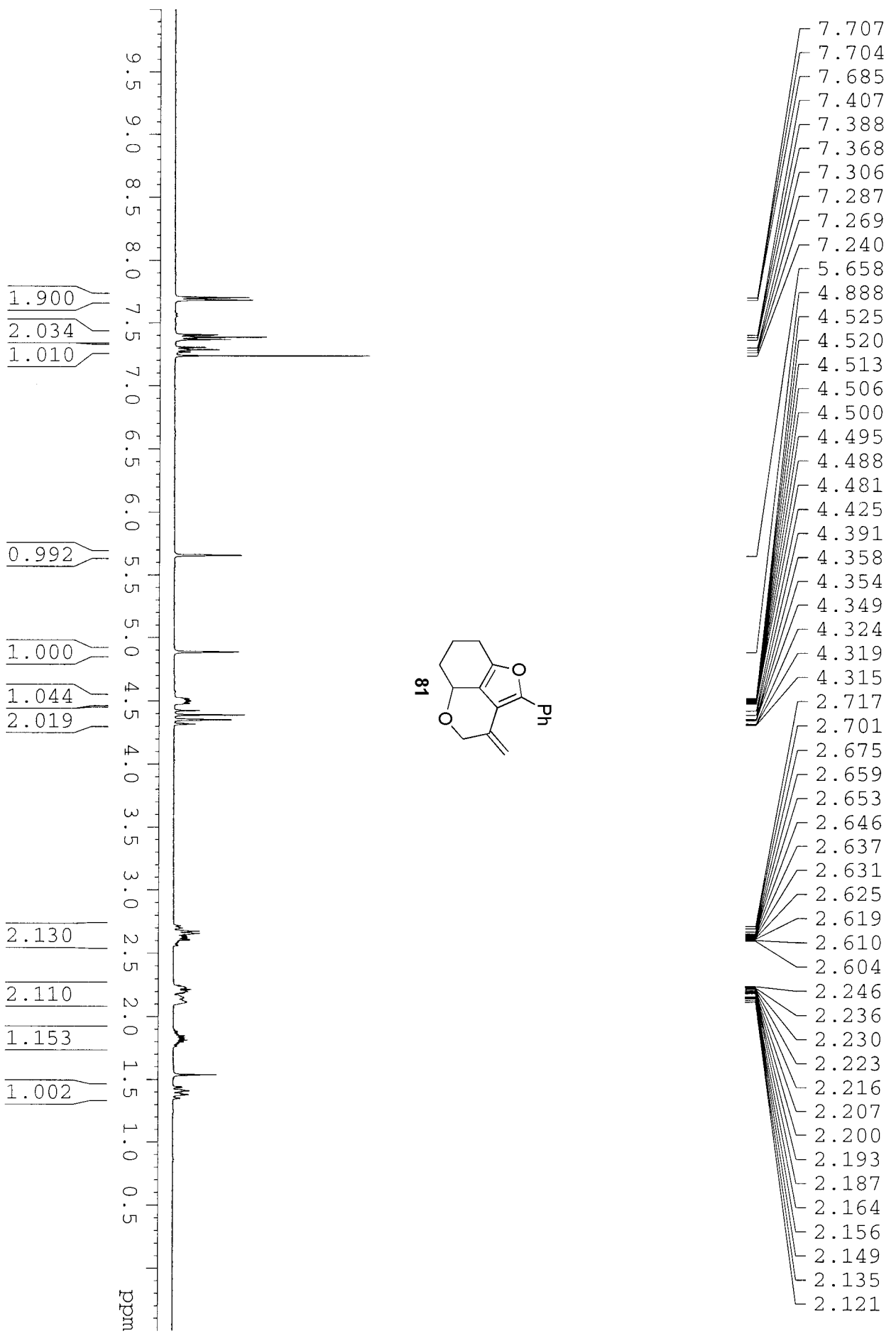


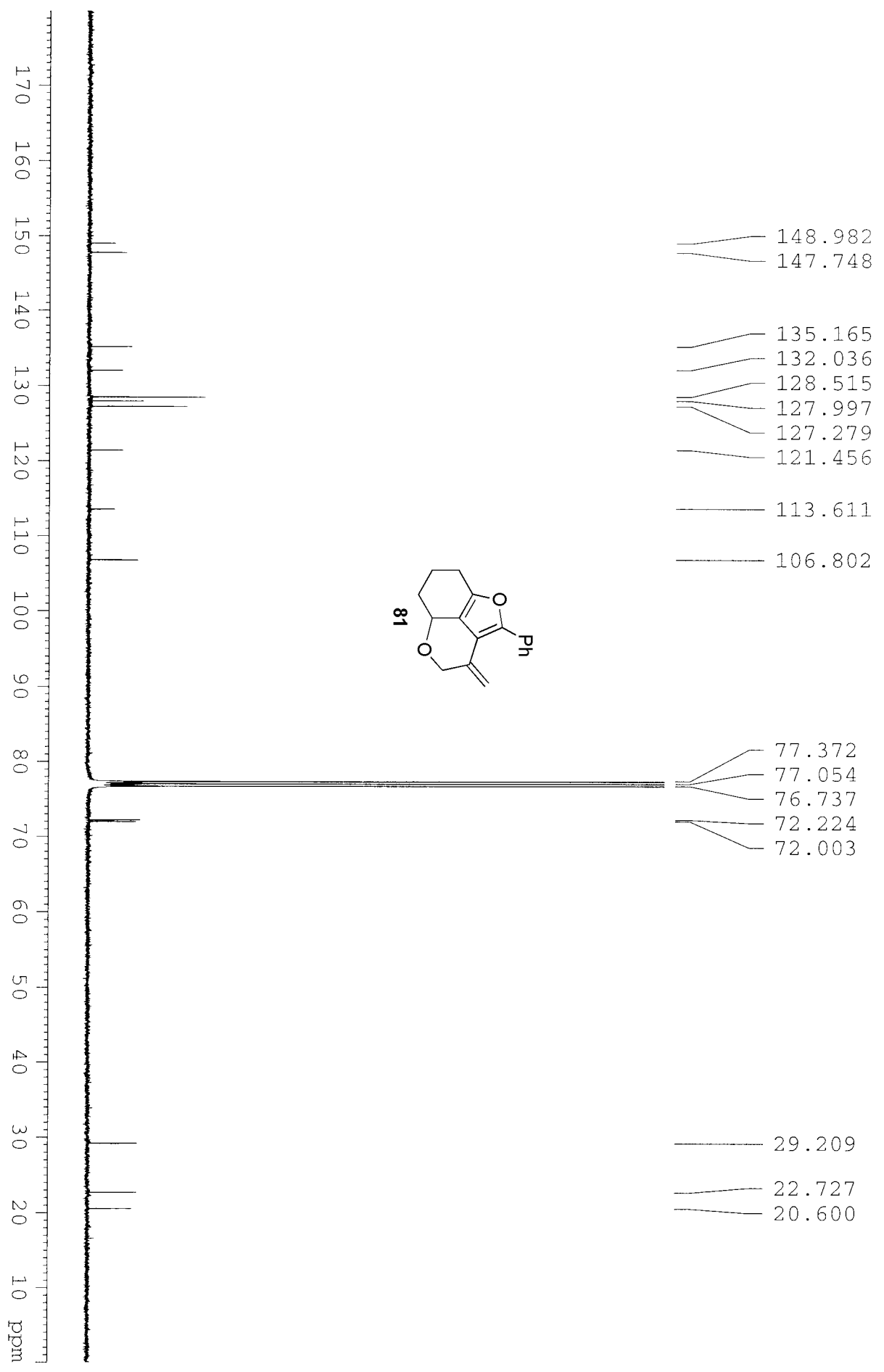



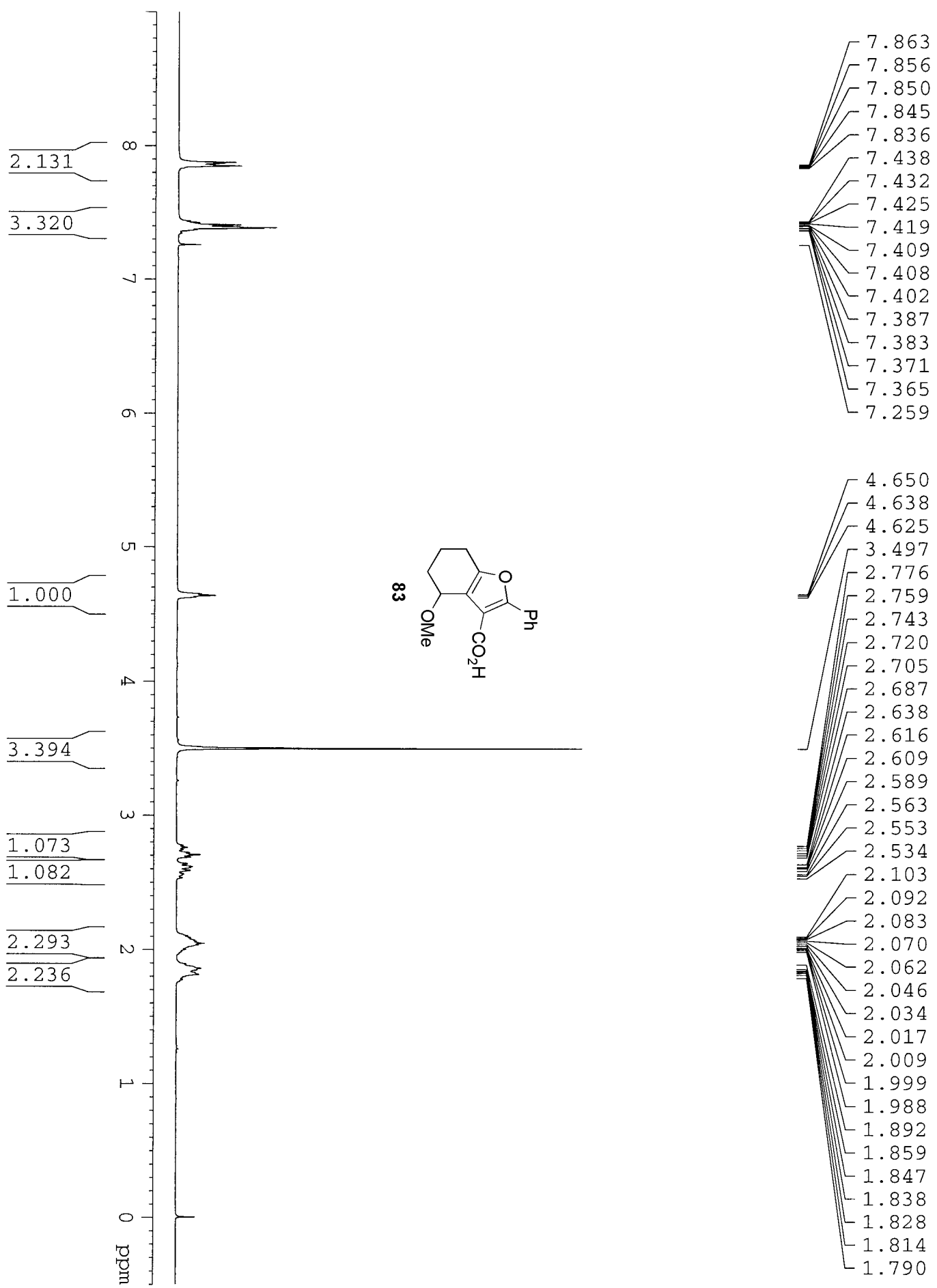

$-2.743$

$-2.720$

$-2.705$

$-2.687$

$-2.638$

- 2.616

$\begin{array}{r}2.609 \\ \hline\end{array}$

2.589
2.563

2.563

$-2.534$

$-2.103$

$-2.092$

$-2.083$

$-2.070$

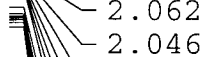

$-2.034$

$-2.017$

2.009

$-1.999$

1.988

1.892

1.859

1.847

L 1.838

1.828

$-1.814$

$-1.790$ 


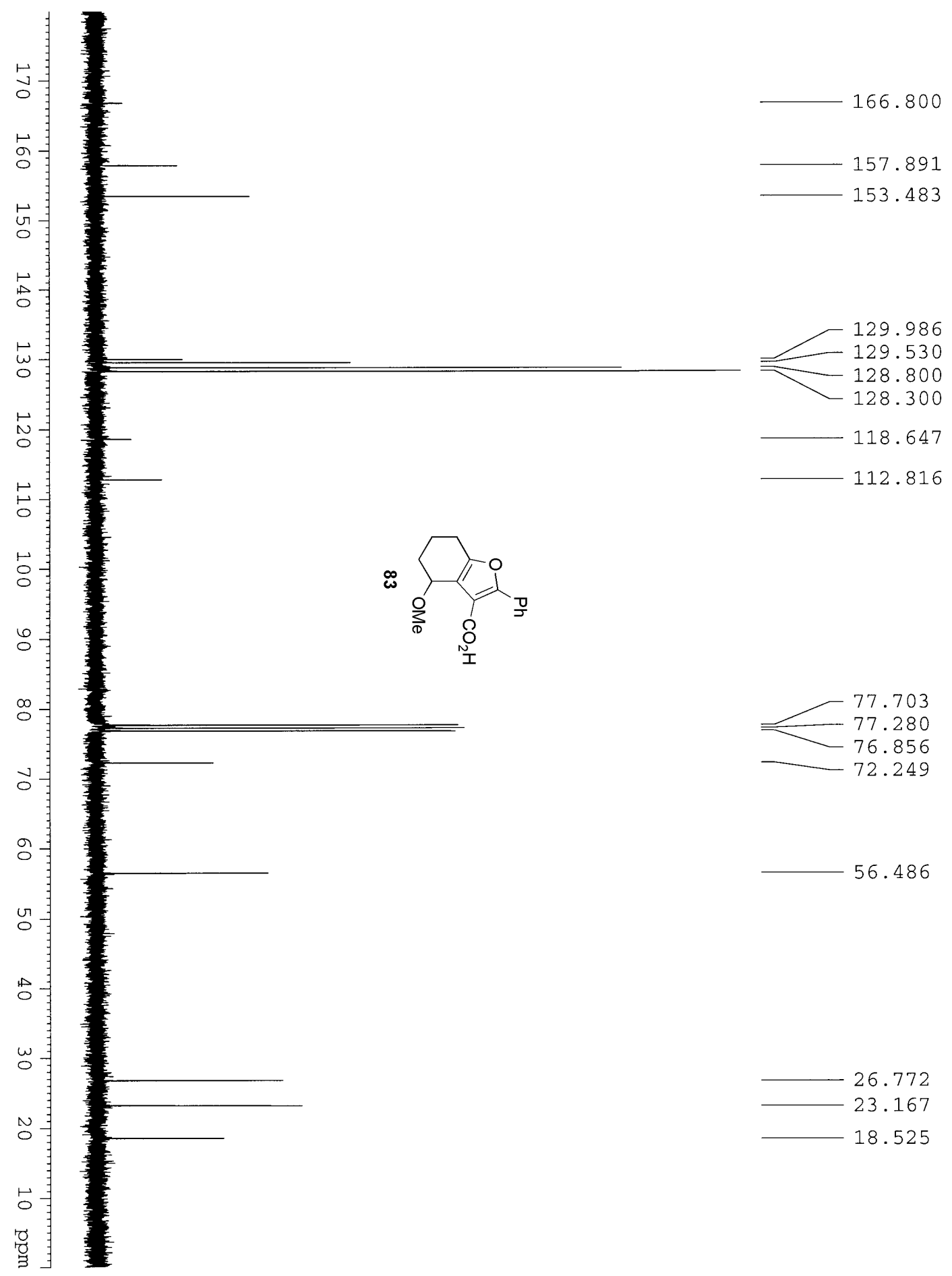



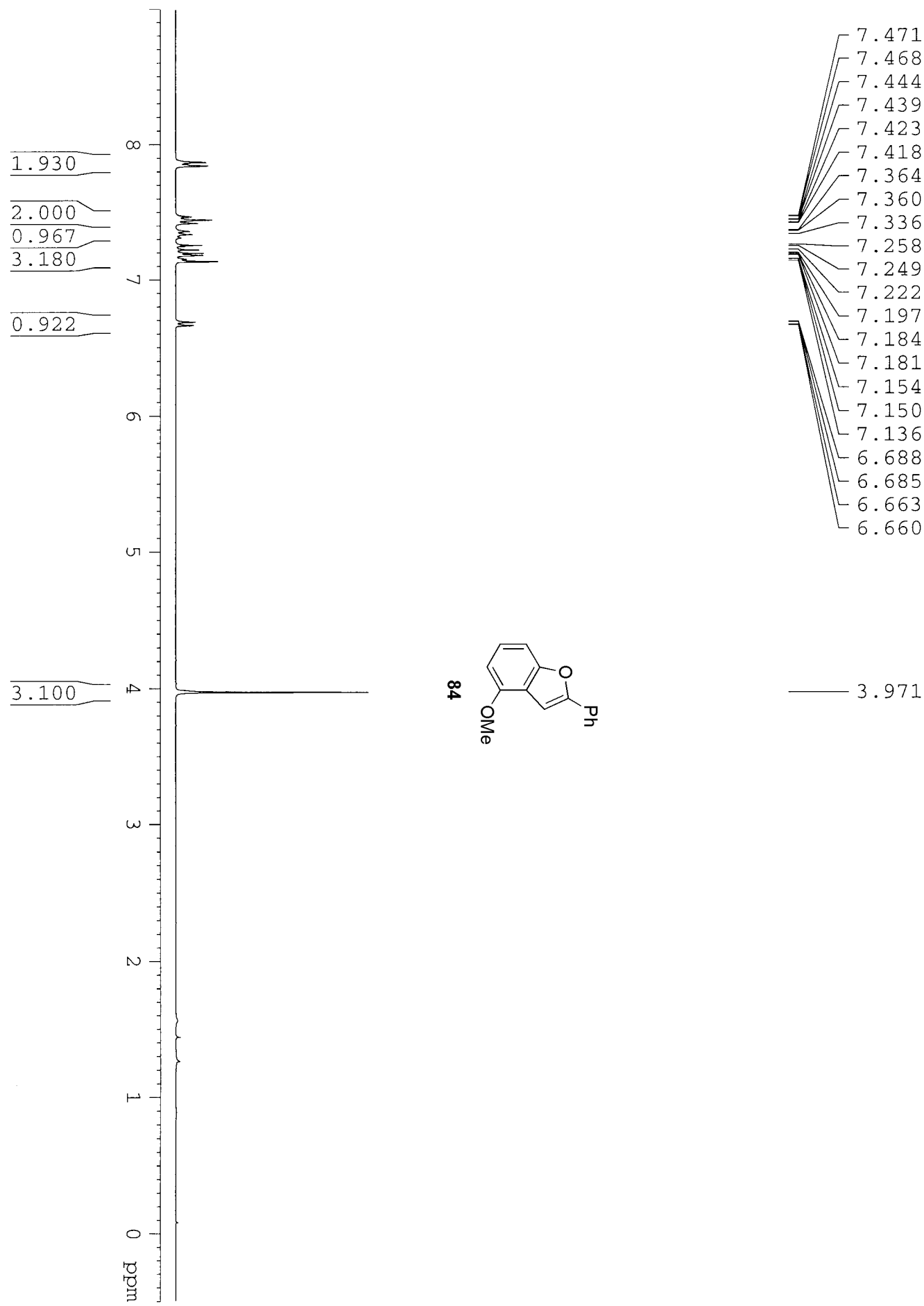

3.971 

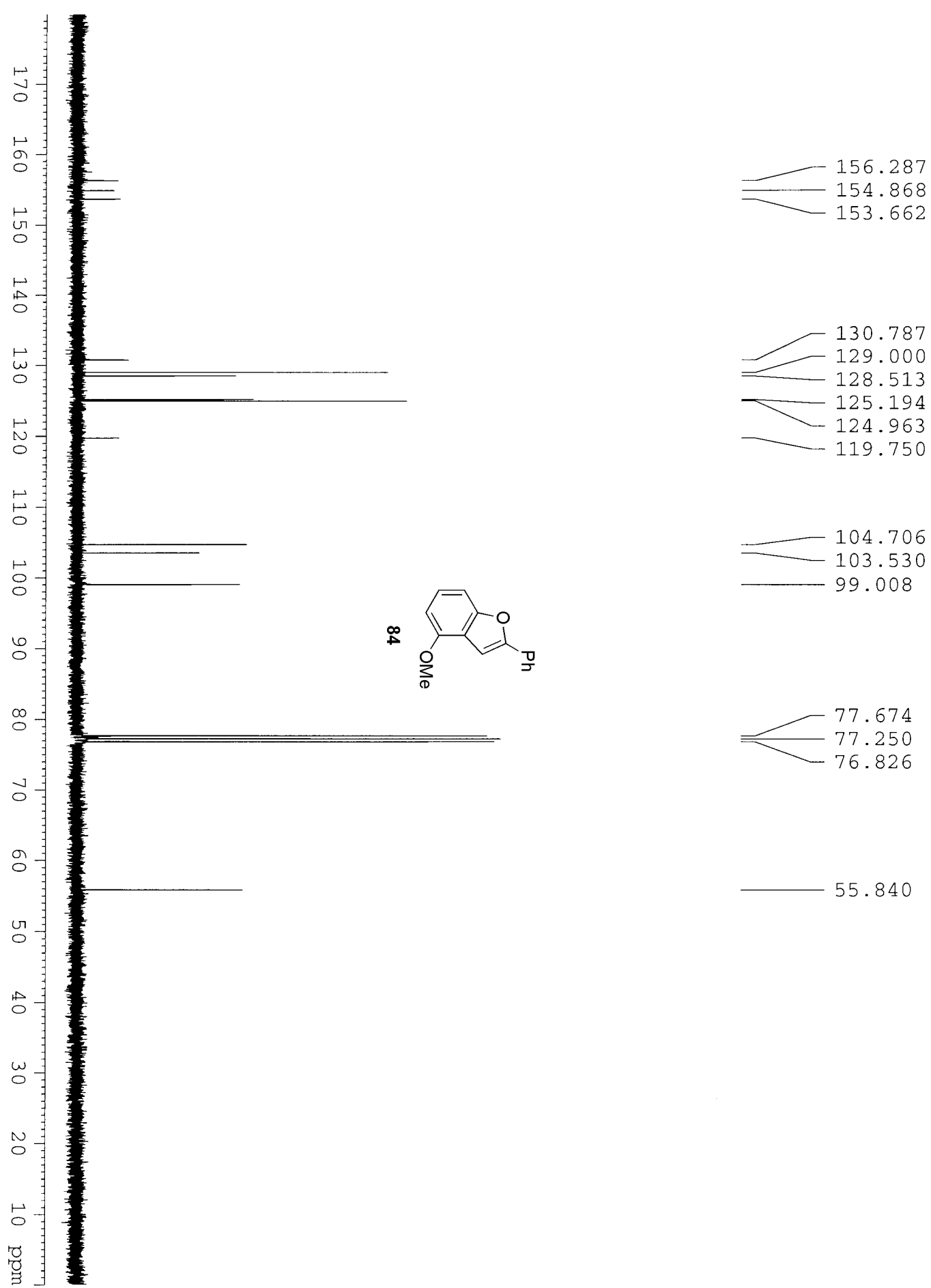

99.008

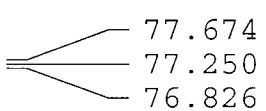

55.840 